\title{
Graph Sparsification, Spectral Sketches, and Faster Resistance Computation, via Short Cycle Decompositions
}

\author{
Timothy Chu \\ Carnegie Mellon \\ tzchu@andrew.cmu .edu \\ Sushant Sachdeva \\ University of Toronto \\ sachdeva@cs.toronto.edu
}

\author{
$\mathrm{Yu}$ Gao \\ Georgia Tech \\ ygao380@gatech.edu \\ Saurabh Sawlani \\ Georgia Tech \\ sawlani@gatech.edu
}

\author{
Richard Peng \\ Georgia Tech \\ rpeng@cc.gatech.edu \\ Junxing Wang \\ Carnegie Mellon \\ junxingw@andrew. cmu . edu
}

May 31, 2018

\begin{abstract}
We develop a framework for graph sparsification and sketching, based on a new tool, short cycle decomposition - a decomposition of an unweighted graph into an edge-disjoint collection of short cycles, plus a small number of extra edges. A simple observation gives that every graph $G$ on $n$ vertices with $m$ edges can be decomposed in $O(m n)$ time into cycles of length at most $2 \log n$, and at most $2 n$ extra edges. We give an $m^{1+o(1)}$ time algorithm for constructing a short cycle decomposition, with cycles of length $n^{o(1)}$, and $n^{1+o(1)}$ extra edges. Both the existential and algorithmic variants of this decomposition enable us to make progress on several open problems in randomized graph algorithms.

1. We present an algorithm that runs in time $m^{1+o(1)} \varepsilon^{-1.5}$ and returns $(1 \pm \varepsilon)$-approximations to effective resistances of all edges, improving over the previous best of $\widetilde{O}\left(\min \left\{m \varepsilon^{-2}, n^{2} \varepsilon^{-1}\right\}\right)$. This routine in turn gives an algorithm to approximate the determinant of a graph Laplacian up to a factor of $(1 \pm \varepsilon)$ in $m^{1+o(1)}+n^{15 / 8+o(1)} \varepsilon^{-7 / 4}$ time.

2. We show existence and efficient algorithms for constructing graphical spectral sketches a distribution over sparse graphs $H$ such that for a fixed vector $\boldsymbol{x}$, we have $\boldsymbol{x}^{\top} \boldsymbol{L}_{H} \boldsymbol{x}=$ $(1 \pm \varepsilon) \boldsymbol{x}^{\top} \boldsymbol{L}_{G} \boldsymbol{x}$ and $\boldsymbol{x}^{\top} \boldsymbol{L}_{H}^{+} \boldsymbol{x}=(1 \pm \varepsilon) \boldsymbol{x}^{\top} \boldsymbol{L}_{G}^{+} \boldsymbol{x}$ with high probability, where $\boldsymbol{L}$ is the graph Laplacian and $\boldsymbol{L}^{+}$is its pseudoinverse. This implies the existence of resistance-sparsifiers with about $n \varepsilon^{-1}$ edges that preserve the effective resistances between every pair of vertices up to $(1 \pm \varepsilon)$.

3. By combining short cycle decompositions with known tools in graph sparsification, we show the existence of nearly-linear sized degree-preserving spectral sparsifiers, as well as significantly sparser approximations of directed graphs. The latter is critical to recent breakthroughs on faster algorithms for solving linear systems in directed Laplacians.

The running time and output qualities of our spectral sketch and degree-preserving (directed) sparsification algorithms are limited by the efficiency of our routines for constructing short cycle decompositions. Improved algorithms for short cycle decompositions will lead to improvements for each of these algorithms.
\end{abstract}




\section{Introduction}

Graph sparsification is a procedure that, given a graph $G$, returns another graph $H$, typically with much fewer edges, that approximately preserves some characteristics of $G$. Graph sparsification originated from the study of combinatorial graph algorithms related to cuts and flows [BK96, EGIN97]. Many different notions of graph sparsification have been extensively studied, for instance, spanners [Che86] approximately preserve pairwise distances, whereas cut-sparsification approximately preserves the sizes of all cuts [BK96]. Spielman and Teng [ST14, ST11b] defined spectral sparsification, a notion that's strictly stronger than a cut-sparsification.

Spectral sparsifiers have found numerous applications to graph algorithms. They are key to fast solvers for Laplacian linear systems [ST14, ST11b, KMP14, KMP11]. Recently they have been used as the sole graph theoretic primitive in graph algorithms including solving linear systems PS14, $\left.\mathrm{KLP}^{+} 16\right]$, sampling random spanning trees $\left[\mathrm{DKP}^{+} 17, \mathrm{DKP}^{+} 17\right.$ ], measuring edge centrality [LZ18, $\left.\mathrm{LPS}^{+} 18\right]$, etc.

For an undirected, weighted graph $G=\left(V, E_{G}, w_{G}\right)$, we recall that the Laplacian of $G, \boldsymbol{L}_{G}$ is the unique symmetric $V \times V$ matrix such that for all $x \in \mathbb{R}^{V}$, we have

$$
x^{\top} \boldsymbol{L}_{G} x=\sum_{(u, v) \in E_{G}} w_{G}(u, v)\left(x_{u}-x_{v}\right)^{2} .
$$

For two positive scalars $a, b$, we write $a \approx_{\varepsilon} b$ if $e^{-\varepsilon} a \leq b \leq e^{\varepsilon} a$. We say the graph $H=\left(V, E_{H}, w_{H}\right)$ is an $\varepsilon$-spectral sparsifier of $G$ if,

$$
\forall x \in \mathbb{R}^{V}, \quad x^{\top} \boldsymbol{L}_{G} x \approx_{\varepsilon} x^{\top} \boldsymbol{L}_{H} x .
$$

Restricting the above definition only to vectors $x \in\{ \pm 1\}^{V}$, one obtains cut sparsifiers. For a graph $G$ with $n$ vertices and $m$ edges, Spielman and Teng gave the first algorithm for constructing spectral sparsifiers with $\widetilde{O}\left(n \varepsilon^{-2}\right)$ edges. Spielman and Srivastava [SS11] proved that one could construct a sparsifier for $G$ by independently sampling $O\left(n \varepsilon^{-2} \log n\right)$ edges with probabilities proportional to their leverage scores in G. Finally, Batson, Spielman, and Srivastava [BSS12] proved that one could construct sparsifiers with $O\left(n \varepsilon^{-2}\right)$ edges, and that this is optimal even for constructing sparsifiers for the complete graph. Recently, Carlson et al. [CKST17] have proved a more general lower bound, proving that one needs $\Omega\left(n \varepsilon^{-2} \log n\right)$ bits to store any data structure that can approximately compute the sizes of all cuts in $G$.

Given the tight upper and lower bounds, it is natural to guess at this point that our understanding of graph sparsification is essentially complete. However, numerous recent works have surprisingly brought to attention several aspects that we do not seem to understand as yet.

1. Are our bounds tight if we relax the requirement in Equation (11) to hold only for a fixed unknown $x$ with high probability? Andoni et al. $\left[\mathrm{ACK}^{+} 16\right]$ define such an object to be a spectral sketch. They also construct a data structure (not a graph) with $\widetilde{O}\left(n \varepsilon^{-1}\right)$ space that is a spectral sketch for $x \in\{ \pm 1\}^{V}$, even though $\Omega\left(n \varepsilon^{-2}\right)$ is a lower bound if one must answer correctly for all $x \in\{ \pm 1\}^{V}$. Building on their work, Jambulapati and Sidford [JS18] showed how to construct such data structures that can answer queries for any $x$ with high probability. A natural question remains open: whether there exist graphs that are spectral sketches with $\widetilde{O}\left(n \varepsilon^{-1}\right)$ edges?

\footnotetext{
${ }^{1}$ The $\widetilde{O}(\cdot)$ notation hides poly $(\log n)$ factors.
} 
2. What if we only want to preserve the effective resistance 2 between all pairs of vertices? Dinitz, Krauthgamer, and Wagner DKW15] define such a graph $H$ as a resistance sparsifier of $G$, and show their existence for regular expanders with degree $\Omega(n)$. They conjecture that every graph admits an $\varepsilon$-resistance sparsifier with $\widetilde{O}\left(n \varepsilon^{-1}\right)$ edges.

3. An $\varepsilon$-spectral sparsifier preserves weighted vertex degrees up to $(1 \pm \varepsilon)$. Do there exist spectral sparsifiers that exactly preserve weighted degrees? Dinitz et al. [DKW15] also explicitly pose a related question - does every dense regular expander contain a sparse regular expander?

4. What about sparsification for directed graphs? The above sparsification notions, and algorithms are difficult to generalize to directed graphs. Cohen et al. $\left[\mathrm{CKP}^{+} 16\right]$ developed a notion of sparsification for Eulerian directed graphs (directed graphs with all vertices having in-degree equal to out-degree), and gave the first almost-linear time algorithm 3 for building such sparsifiers. However, their algorithm is based on expander decomposition, and isn't as versatile as the importance sampling based sparsification of undirected graphs [SS11]. Is there an easier approach to sparsifying Eulerian directed graphs?

5. There is an ever-growing body of work on the algorithmic applications of graph sparsification [Spi10, Ten10, BSST13, Ten16]. Could the above improved guarantees lead to even faster algorithms for some of these problems? Two problems of significant interest include estimating determinants $\left[\mathrm{DKP}^{+} 17\right]$ and sampling random spanning trees $\left[\mathrm{DKP}^{+} 17, \mathrm{DPPR} 17, \mathrm{Sch} 17\right]$.

\subsection{Our Contributions}

In this paper, we develop a framework for graph sparsification based on a new graph-theoretic tool we call short cycle decomposition. Informally, a short cycle decomposition of a graph $G$ is a decomposition into a sparse graph, and several cycles of short length. We use our framework to give affirmative answers to all the challenges in graph sparsification discussed in the previous section. Specifically:

1. We show that every graph $G$ has a graph $H$ with $\widetilde{O}\left(n \varepsilon^{-1}\right)$ edges that is an $\varepsilon$-spectral-sketch for $G$. The existence of such graphic spectral-sketches was not known before. Moreover, we give an algorithm to construct an $\varepsilon$-spectral-sketch with $n^{1+o(1)} \varepsilon^{-1}$ edges in $m^{1+o(1)}$ time. In addition, $H$ is also a spectral-sketch for $\boldsymbol{L}_{G}^{+}$.

2. We show every graph $G$ has an $\varepsilon$-resistance sparsifier with $\widetilde{O}\left(n \varepsilon^{-1}\right)$ edges, affirmatively answering the question raised by Dinitz et al. [DKW15]. We also give an algorithm to construct $\varepsilon$-resistance sparsifiers with $n^{1+o(1)} \varepsilon^{-1}$ edges in $m^{1+o(1)}$ time.

3. We show that every graph has an $\varepsilon$-spectral sparsifier with $\widetilde{O}\left(n \varepsilon^{-2}\right)$ edges that exactly preserves the weighted-degrees of all vertices. It follows that every dense regular expander contains a sparse (weighted) regular expander. Before our work, it was not known if there exist sparse degree-preserving sparsifiers (even for cut sparsifiers).

4. We show that short cycle decompositions can be used for constructing sparse spectral approximations for Eulerian directed graphs under the notion of spectral approximation given by Cohen et al. $\left[\mathrm{CKP}^{+} 16\right]$ for Eulerian directed graphs (see 3.2 for definition). We show that

\footnotetext{
${ }^{2}$ The effective resistance between a pair $u, v$ is the voltage difference between $u, v$ if we consider the graph as an electrical network with every edge of weight $w_{e}$ as a resistor of resistance $\frac{1}{w_{e}}$, and we send one unit of current from $u$ to $v$.

${ }^{3} \mathrm{An}$ algorithm is said to be almost-linear time if it runs in $m^{1+o(1)}$ time on graphs with $m$ edges.
} 
short-cycle decompositions are sufficient for sparsifying Eulerian directed graphs, and prove that every directed Eulerian graph has a spectral approximation with $O\left(n \varepsilon^{-2} \log ^{4} n\right)$ edges.

5. We build on our spectral-sketches, to give an algorithm for estimating the effective resistances of all edges up to a factor of $(1 \pm \varepsilon)$ in $m^{1+o(1)} \varepsilon^{-1.5}$ time. The previous best results for this algorithm were $\widetilde{O}\left(m \varepsilon^{-2}\right)$ [SS11] and $\widetilde{O}\left(n^{2} \varepsilon^{-1}\right)$ [JS18].

Incorporating this result into the work of Durfee et al. [DPPR17] gives an $m^{1+o(1)}+n^{15 / 8+o(1)} \varepsilon^{-7 / 4}$ time algorithm for approximating the determinant of a $\boldsymbol{L}$ (rather, $\boldsymbol{L}$ after deleting the last row and column, which is the number of spanning trees in a graph), up to a factor of $(1 \pm \varepsilon)$. The previous best algorithm for this problem ran in time $\widetilde{O}\left(n^{2} \varepsilon^{-2}\right)\left[\mathrm{DKP}^{+} 17\right]$.

As a key component of all our results, we present efficient algorithms for constructing short cycle decompositions. From a bird's eye view, the key advantage provided by short cycle decompositions for all the above results, is that they allow us to sample edges in a coordinated manner so to preserve weighted vertex degrees exactly.

Definition 1.1. An $(\widehat{m}, L)$-short cycle decomposition of an unweighted undirected graph $G$, decomposes $G$ into several edge-disjoint cycles, each of length at most $L$, and at most $\widehat{m}$ edges not in these cycles.

The existence of such a decomposition with $\widehat{m}(m, n) \leq 2 n$ and $L(m, n) \leq 2 \log n$ is a simple observation. We repeatedly remove vertices of degree at most 2 from the graph, along with their incident edges (removing at most $2 n$ edges in total). If the remaining graph has no cycle of length at most $2 \log n$, a breadth-first search tree of depth $\log n$ starting from any remaining vertex will contain more than $n$ vertices, a contradiction. This can be implemented as an $O(m n)$ time algorithm to find a $(2 n, 2 \log n)$-short cycle decomposition, which in turn implies a similar running time for all the existential results above. Finding such decompositions faster is a core component of this paper: we give an algorithm that constructs an $\left(n^{1+o(1)}, n^{o(1)}\right)$-short cycle decomposition of a graph in $m^{1+o(1)}$ time.

Organization. To keep this section brief, we defer the formal definitions and theorem statements to the overview of the work (Section 3), after defining a few necessary preliminaries in Section 2 , We start with degree-preserving spectral sparsifiers in Section 4, and then give the algorithm for sparsification of Eulerian directed graphs (Section [5). Next, we present the construction of spectral-sketches and resistance sparsifiers in Section 6. followed by our algorithm for estimating effective resistances for all edges in Section 7. Finally, we give our almost-linear time algorithm for constructing a short cycle decomposition in Section 8 ,

\section{Preliminaries}

A square symmetric $n \times n$ matrix $\boldsymbol{M}$ is positive semi-definite (PSD), denoted $\boldsymbol{M} \succeq 0$, if for all $\boldsymbol{x} \in \mathbb{R}^{n}$, we have $\boldsymbol{x}^{\top} \boldsymbol{M} \boldsymbol{x} \geq 0$. For two matrices $\boldsymbol{M}_{1}, \boldsymbol{M}_{2}$, we write $\boldsymbol{M}_{1} \succeq \boldsymbol{M}_{2}$ if for all $\boldsymbol{x} \in \mathbb{R}^{n}, \boldsymbol{x}^{\top} \boldsymbol{M}_{1} \boldsymbol{x} \geq \boldsymbol{x}^{\top} \boldsymbol{M}_{2} \boldsymbol{x}$, or equivalently $\boldsymbol{M}_{1}-\boldsymbol{M}_{2} \succeq 0$.

For $\varepsilon \geq 0$, and two positive real numbers $a, b$, we write $a \approx_{\varepsilon} b$ to express $e^{-\varepsilon} a \leq b \leq e^{\varepsilon} a$. Observe that $a \approx_{\varepsilon} b$ if $b \approx_{\varepsilon} a$. For two PSD matrices $\boldsymbol{M}_{1}, \boldsymbol{M}_{2}$, we write $\boldsymbol{M}_{1} \approx_{\varepsilon} \boldsymbol{M}_{2}$ if for all $\boldsymbol{x} \in \mathbb{R}^{n}, \boldsymbol{x}^{\top} \boldsymbol{M}_{1} \boldsymbol{x} \approx_{\varepsilon} \boldsymbol{x}^{\top} \boldsymbol{M}_{2} \boldsymbol{x}$.

Fact 2.1. For any PSD $\boldsymbol{M}_{1}, \boldsymbol{M}_{2}, \boldsymbol{M}_{3}$ and $\varepsilon_{1}, \varepsilon_{2} \geq 0$, if we have $\boldsymbol{M}_{1} \approx_{\varepsilon_{1}} \boldsymbol{M}_{2}$ and $\boldsymbol{M}_{2} \approx_{\varepsilon_{2}} \boldsymbol{M}_{3}$, then $M_{1} \approx_{\varepsilon_{1}+\varepsilon_{2}} M_{3}$. 
For two graphs $G_{1}, G_{2}$, we often abuse notation to write $G_{1} \succeq G_{2}$ to mean $\boldsymbol{L}_{G_{1}} \succeq \boldsymbol{L}_{G_{2}}$ and $G_{1} \approx_{\varepsilon} G_{2}$ to mean $\boldsymbol{L}_{G_{1}} \approx_{\varepsilon} \boldsymbol{L}_{G_{2}}$.

For any PSD matrix $\boldsymbol{M}$, we let $\boldsymbol{M}^{+}$denote the Moore-Penrose pseudoinverse of $\boldsymbol{M}$. Thus, if $\boldsymbol{M}$ has an eigenvalues $0 \leq \lambda_{1} \leq \lambda_{2} \leq \ldots \leq \lambda_{n}$, with unit-norm eigenvectors $v_{1}, v_{2}, \ldots, v_{n}$ respectively, we have $\boldsymbol{M}=\sum_{i} \lambda_{i} v_{i} v_{i}^{\top}$, and $\boldsymbol{M}^{+}=\sum_{\lambda_{i}>0} \frac{1}{\lambda_{i}} v_{i} v_{i}^{\top}$. Similarly, we have $\boldsymbol{M}^{1 / 2}=\sum_{i} \sqrt{\lambda_{i}} v_{i} v_{i}^{\top}$, and $M^{+/ 2}=\sum_{\lambda_{i}>0} \frac{1}{\sqrt{\lambda_{i}}} v_{i} v_{i}^{\top}$.

Our notion of approximation is preserved under inverses:

Fact 2.2. For any PSD $\boldsymbol{M}_{1}$ and $\boldsymbol{M}_{2}$, and any error $\varepsilon>0$, we have $\boldsymbol{M}_{1} \approx_{\varepsilon} \boldsymbol{M}_{2}$ if and only if $M_{1}^{+} \approx_{\varepsilon} M_{2}^{+}$.

For any $u$, we let $\chi_{u}$ denote the vector such that the $u^{\text {th }}$ coordinate is 1 , and all other coordinates are 0 . We let $\chi_{u v}=\chi_{u}-\chi_{v}$. For any edge $e=(u, v)$ in a connected graph $G$, the effective resistance of $e$ is defined as $R_{\text {eff }}(e)=\chi_{u v}^{\top} \boldsymbol{L}_{G}^{+} \chi_{u v}$. For a directed graph $\vec{G}$, its directed Laplacian $\boldsymbol{L}_{\vec{G}}$, can be defined as $\sum_{e=u \rightarrow v} \chi_{u v}^{\top} \chi_{u}$.

All logarithms throughout the paper are with base 2. Unless mentioned, we assume that our input graph $G$ has $m$ edges and $n$ vertices. Throughout the paper, we consider graphs with positive integral weights on the edges. Whenever we say the weights are poly bounded, we assume they are bounded by $n^{O(1)}$. The expression with high-probability means with probability larger than $1-\frac{1}{n^{\Omega(1)}}$.

\section{Overview}

There are 4 major approaches to date towards graph sparsification: expander partitioning ST11b, $\mathrm{ACK}^{+}$16, JS18], importance sampling [BK96, SS11, KLP12], potential function based BSS12, ALO15, LS15, LS17], and spanners based, which use sampling via matrix concentration [KP12, Kou14, KPPS17]. A survey of these approaches can be found in [BSST13].

We present a framework for graph sparsification built on short cycle decomposition that merges several ideas from the importance-sampling and spanners based approaches. Before giving an overview of the results in our paper, we first present an alternative algorithm for the classic graph sparsification result of Spielman and Srivastava [SS11]. This will be quite useful since our algorithms for constructing degree-preserving sparsifiers and sparsifying Eulerian directed graphs are immediately built on the following algorithm, and degree-preserving sparsification is a key idea underlying all our remaining results.

Say we have a graph $G(V, E, w)$ with $m$ edges and $n$ edges. We start by expressing $\boldsymbol{L}_{G}=$ $\sum_{e \in E} w_{e} \boldsymbol{L}_{e}$, where for edge $e=(u, v), \boldsymbol{L}_{e}=\chi_{u v} \chi_{u v}^{\top}$. We can re-write this as

$$
\boldsymbol{\Pi}=\sum_{e} w_{e} \boldsymbol{L}_{G}^{+/ 2} \boldsymbol{L}_{e} \boldsymbol{L}_{G}^{+/ 2}
$$

where $\boldsymbol{\Pi}$ is the projection orthogonal to the all ones vector. Given a subset of edges $E^{\prime} \subseteq E$, we draw a random graph $H$ as follows, independently for every edge $e \in E^{\prime}$, we include it in $H$ with probability $1 / 2$ and weight $2 w_{e}$. Otherwise, we delete the edge $e$. All edges $e \in E \backslash E^{\prime}$ are included in $H$ with weight $w_{e}$. Observe that the expectation of $\boldsymbol{L}_{H}$ is $\boldsymbol{L}_{G}$.

It follows from standard concentration results for sums of matrix random variables that if for each edge $e$ in $E^{\prime}$, the norm $\left\|w_{e} \boldsymbol{L}_{G}^{+/ 2} \boldsymbol{L}_{e} \boldsymbol{L}_{G}^{+/ 2}\right\|$ is bounded (up to constants) by $\frac{\varepsilon^{2}}{\log n}$, then with high probability, $\boldsymbol{L}_{H} \approx_{\varepsilon} \boldsymbol{L}_{G}$. 
Now, observe that $\left\|w_{u v} \boldsymbol{L}_{G}^{+/ 2} \boldsymbol{L}_{u v} \boldsymbol{L}_{G}^{+/ 2}\right\|=w_{e} \chi_{u v} \boldsymbol{L}_{G}^{+} \chi_{u v}=w_{u v} R_{\mathrm{eff}}(u, v)$ (this is defined as the leverage score of the edge $u v)$. A simple trace argument implies $\sum_{e} w_{e} R_{\text {eff }}(e)=\frac{n-1}{m}$, and hence at least half the edges satisfy $w_{e} R_{\mathrm{eff}}(e) \leq \frac{2 n}{m}$. Letting these edges with low leverage score be the set of edges $E^{\prime}$ we toss random coins for, we obtain that $\boldsymbol{L}_{H} \approx \sqrt{\frac{2 n}{m} \log n} \boldsymbol{L}_{G}$. Moreover, in expectation, $H$ has at most $\frac{3}{4} m$ edges.

We can repeat the above sparsification roughly $O(\log n)$ times to go down to $O\left(n \varepsilon^{-2} \log n\right)$ edges, at each step sparsifying the graph just obtained. By Fact 2.1, the final approximation error is given by the sum of the error at each sparsification step. Since the number of edges is going down geometrically, the error is increasing geometrically, and hence is dominated by the error at the last step, yielding that the final graph is an $O\left(\sqrt{\frac{2 n \log n}{n \varepsilon^{-2} \log n}}\right)=O(\varepsilon)$ spectral-sparsifier for $G$.

In order to implement this algorithm efficiently, we need to estimate effective resistances for the edges. For the above algorithm, constant factor estimates of the effective resistances suffice (at the cost of changing the constants involved). Spielman and Srivastava [SS11] showed that one can obtain constant factor estimates for all the edges together in $\widetilde{O}(m)$ time, resulting in a complete running time of $\widetilde{O}(m)$ for the above sparsification algorithm.

\subsection{Degree Preserving Spectral Sparsification}

Now, we adapt the above algorithm to leverage a short-cycle decomposition of the graph. Short cycles permit us to sample correlated edges in the graph while still keeping each random sample small in spectral norm. We first use this approach to construct degree-preserving spectral sparsifiers.

We first formally define a degree-preserving sparsifier.

Definition 3.1 (Degree-Preserving Sparsifier). A graph $H\left(V, E^{\prime}\right)$ is said to be a degree-preserving E-sparsifier of $G(V, E)$ if

1. for every $x \in \mathbb{R}^{V}$, we have, $x^{\top} \boldsymbol{L}_{G} x \approx_{\varepsilon} x^{\top} \boldsymbol{L}_{H} x$, and

2. every vertex $u \in V$ has the same weighted degree in $G$ and $H$, i.e., $\sum_{v} w_{u, v}^{G}=\sum_{v} w_{u, v}^{H}$.

Given the above algorithm for usual graph sparsification, the main obstacle is that at each sparsification step, the weighted degrees are not preserved. This is where we require our key tool, a short cycle decomposition, which we now formally define.

Definition 3.2. For an undirected unweighted graph $G(V, E)$, we say that $\left\{C_{1}, C_{2}, \ldots\right\}$ is a an $(\widehat{m}, L)$-short cycle decomposition, if $C_{1}, C_{2}, \ldots$ are edge-disjoint cycles in $G$, each $C_{i}$ is a cycle of length at most $L$, and $\left|E \backslash \bigcup_{i} C_{i}\right| \leq \widehat{m}$.

Assuming that we have an efficient algorithm for constructing an $(\widehat{m}, L)$-short cycle decomposition of any given graph, we show the following theorem.

Theorem 3.3. Given $\varepsilon \in(0,1]$, every undirected graph $G$ with poly-bounded weights has a degreepreserving $\varepsilon$-sparsifier with $O\left(n \varepsilon^{-2} \log ^{2} n\right)$ edges. The algorithm DEGREEPRESERVINGSPARSIFY, given our short cycle decomposition algorithm, takes in a graph $G$ and runs in time $m^{1+o(1)}$ and returns a degree-preserving $\varepsilon$-sparsifier of $G$ with $n^{1+o(1)} \varepsilon^{-2}$ edges.

The following is a brief description of our degree-preserving sparsification algorithm. 


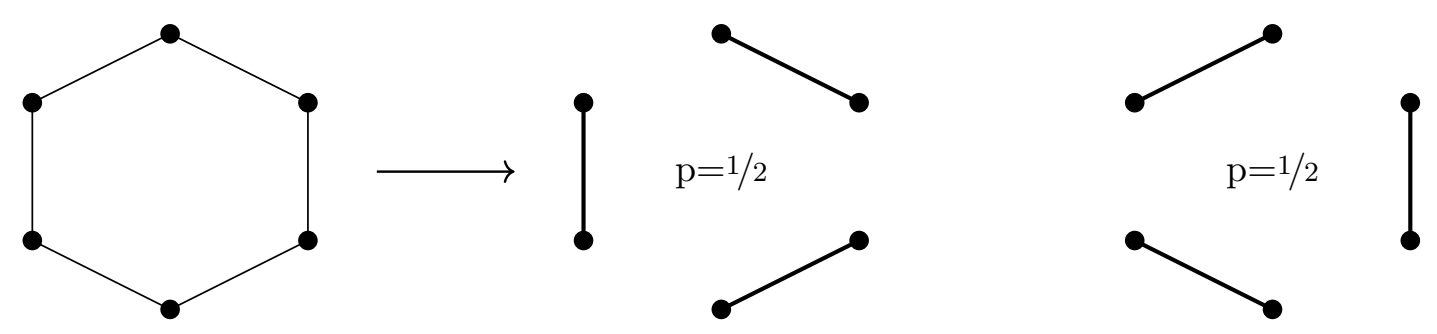

Figure 1: Sampling alternate edges in short cycles for degree-preserving sparsification. The thick edges are double the weights of the thin edges.

Assume first that our graph $G$ is an unweighted graph that has been given to us as the union of disjoint cycles of even length. We sample a random graph $H$ as follows. For each cycle independently, we index the edges in order start from an arbitrary vertex, and perform the following correlated sampling procedure: with probability $1 / 2$, we keep only the even indexed edges with weight 2 , and with probability $1 / 2$, we keep only the odd indexed edges with weight 2 (see Figure 1). Observe that $H$ has half as many edges as $G$, and has exactly the same weighted degrees as $G$. In order to apply matrix concentration, we need to ensure that for each cycle $C$, the norm $\left\|\boldsymbol{L}_{G}^{+/ 2} \boldsymbol{L}_{C} \boldsymbol{L}_{G}^{+/ 2}\right\|$ is at most $\frac{\varepsilon^{2}}{\log n}$, where $\boldsymbol{L}_{C}$ is the Laplacian of the cycle $C$. This norm is easily upper bounded by $\sum_{e \in C} R_{\mathrm{eff}}(e)$.

If instead, $G$ was any arbitrary unweighted graph, we move all the edges with $R_{\text {eff }} \geq \frac{2 n}{m}$ to $H$. Again, by averaging, we still have at least $m / 2$ edges remaining. Now, we greedily pick a bi-partition of the vertices of $G$ such that at least half the remaining edges are crossing the cut. We add all the non-crossing edges to $H$. Now, we utilize an $(\widehat{m}, L)$-short cycle decomposition of $G$. Thus, all but $\widehat{m}$ edges of $G$ are partitioned into cycles of length at most $L$. Observe that all the cycle edges crossing the bi-partition, at least must now be in even cycles, each with total $R_{\text {eff }}$ bounded by $\frac{2 n L}{m}$. Now, independently for each cycle, we pick even or odd edges with probability $1 / 2$, and add them to $H$ with weight 2 . Assuming $m \geq 8 \widehat{m}, H$ has at most $\frac{15}{16} m$ edges, the same weighted degree as $G$, and with high probability $H \approx \sqrt{\frac{2 n L}{m} \log n} G$.

Note that re-framing original sparsification into an algorithm for reducing the edges by a constant fraction is crucial for this algorithm. We are only able to reduce the edges in a cycle by half. Further, the cycle decomposition of the graph will necessarily change with every iteration.

For starting with a weighted graph with poly-bounded weights, we can use the binary representation of the edge weight to split each edge into $O(\log n)$ edges, each with a weight that's a power of 2. Now, repeating the above procedure as before, we can construct a degree-preserving $\varepsilon$-sparsifier for $G$ with roughly $\widehat{m} \log n+n \varepsilon^{-2} L \log n$ edges. Using the $O(\log n)$ length short-cycle decomposition, this gives roughly $n \varepsilon^{-2} \log ^{2} n$ edges.

\subsection{Sparsification of Eulerian Directed Graphs}

Now, we can take a very similar approach to sparsifying Eulerian directed graphs. This is a primitive introduced in $\left[\mathrm{CKP}^{+} 16\right]$, and is at the core of recent developments in fast solvers for linear systems in directed Laplacians $\left[\mathrm{CKP}^{+} 16, \mathrm{CKP}^{+} 17, \mathrm{Kyn} 17\right]$. In contrast to undirected graphs, it has been significantly more challenging to give an appropriate notion of approximation for directed graphs (see Section 5 for the definition of Laplacian $\boldsymbol{L}_{\vec{G}}$ of a directed graph $G$ ). Cohen et al. [CKP ${ }^{+}$]] 
showed that for the purpose of solving linear systems in Eulerian directed graphs, one such useful notion is to say $\vec{H}$-approximates $\vec{G}$ if

$$
\left\|\boldsymbol{L}_{G}^{+/ 2}\left(\boldsymbol{L}_{\vec{H}}-\boldsymbol{L}_{\vec{G}}\right) \boldsymbol{L}_{G}^{+/ 2}\right\| \leq \varepsilon
$$

where $G$ is the undirectification of $G$, i.e., the underlying undirected graph of $\vec{G}$ with edge-weights halved. In the case where $\vec{G}$ is Eulerian, $\boldsymbol{L}_{G}=\frac{1}{2}\left(\boldsymbol{L}_{\vec{G}}+\boldsymbol{L}_{\vec{G}}^{\top}\right)$.

The key obstacle in sparsifying Eulerian directed graphs is to sample directed subgraphs $\vec{H}$ that are Eulerian since independent sampling cannot provide us with such precise control on the degrees. The work of Cohen et al. $\left[\mathrm{CKP}^{+} 17\right]$ fixed this locally by modifying the diagonal in $\boldsymbol{L}_{\vec{H}}$ in order to make the sampled graph Eulerian. This approach induces an error in $\boldsymbol{L}_{\vec{H}}$ of the order of $\varepsilon \boldsymbol{D}_{\vec{G}}$ where $\boldsymbol{D}$ is the diagonal out-degree matrix for $\vec{G}$. In order for this error to be small relative to $\boldsymbol{L}_{\vec{G}}, \vec{G}$ must be an expander. Hence, the need of expander partitioning in their approach.

However, as we saw above, a short cycle decomposition allows us to perform correlated sampling on edges with precise control on the degrees. For sampling directed graphs, consider a single cycle where the edges may have arbitrary direction (see Figure 2). With probability $1 / 2$, we sample the edges in clockwise-direction, and with probability $1 / 2$, we sample the edges in the anti-clockwise direction. In either case, we double the weights of the sampled edges. Observe that for each vertex, the difference between the outgoing and incoming degrees is preserved exactly. Hence, if we started with an Eulerian directed graph, we end up with an Eulerian directed graph. Moreover, in expectation, we only keep half the edges of the cycle.

We can now basically follow the algorithm for degree-preserving sparsification. We treat the graph as undirected for all steps except for sampling edges from a cycle. In particular, the cycle decomposition is found in the corresponding undirected graph. Using the above approach for sampling edges from each cycle, we can sample an Eulerian directed graph, that has a constant fraction fewer edges in expectation. Since the matrices involved are no longer symmetric, we invoke concentration bounds for rectangular matrices to obtain

$$
\left\|\boldsymbol{L}_{G}^{+/ 2}\left(\boldsymbol{L}_{\vec{H}}-\boldsymbol{L}_{\vec{G}}\right) \boldsymbol{L}_{G}^{+/ 2}\right\| \leq O\left(\sqrt{\frac{n L^{3} \log n}{m}}\right) .
$$

Now, repeating this sparsification procedure, and observing that this notion of approximation error also composes, we obtain an Eulerian directed graph $\vec{H}$ that $\varepsilon$-approximates $\vec{G}$ with roughly $\widehat{m} \log n+n \varepsilon^{-2} L^{3} \log n$ edges. Again, using the naive cycle decomposition, this is $O\left(n \varepsilon^{-2} \log ^{4} n\right)$ edges.

Theorem 3.4. Given $\varepsilon \in(0,1]$, for every Eulerian directed graph $\vec{G}$, we can find in $\widetilde{O}(m n)$ time an Eulerian directed graph $\vec{H}$ with $O\left(n \varepsilon^{-2} \log ^{4} n\right)$ edges, that $\varepsilon$-approximates $\vec{G}$.

This shows the existence of sparsifiers for Eulerian graphs with fewer edges than the nearlylinear sized ones constructed in Cohen et al. $\left[\mathrm{CKP}^{+} 17\right]$. More importantly, it shows that approaches based on importance sampling, which work well on undirected graphs, can work in the more general directed settings as well. However, the high costs of computing short cycle decompositions in this paper means this does not lead to faster asymptotic running times in the applications - we believe this is an interesting direction for future work. 


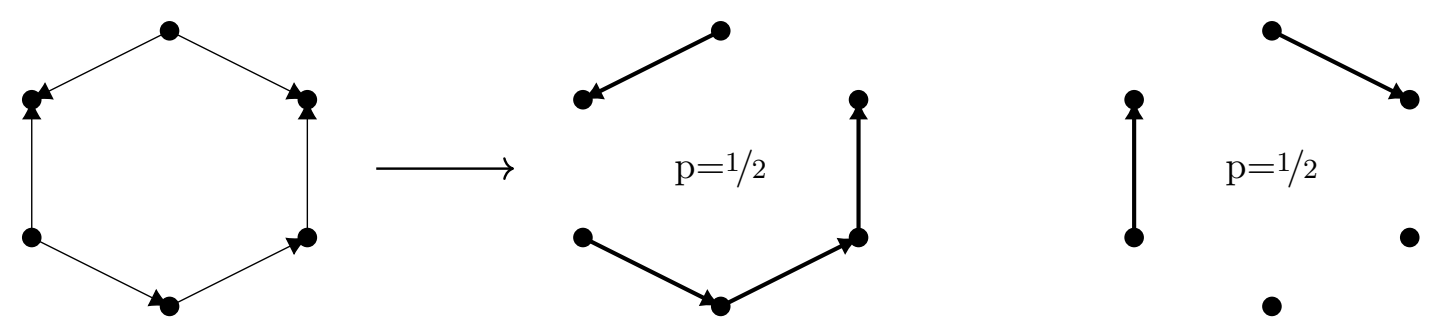

Figure 2: Sampling edges along a random direction in short cycles for sparsification of Eulerian directed graphs. The thick edges are double the weights of the thin edges.

\subsection{Graphical Spectral Sketches and Resistance Sparsifiers}

We define a graphical spectral sketch as follows:

Definition 3.5 (Graphical Spectral Sketch). Given a graph $G(V, E)$, a distribution $\mathcal{H}$ over random graphs $H\left(V, E^{\prime}\right)$ is said to be a graphical $\varepsilon$-spectral sketch for $G$, if for any fixed $\boldsymbol{x} \in \mathbb{R}^{V}$, with high probability, over the sample $H \sim \mathcal{H}$, we have $\boldsymbol{x}^{\top} \boldsymbol{L}_{G} \boldsymbol{x} \approx_{\varepsilon} \boldsymbol{x}^{\top} \boldsymbol{L}_{H} \boldsymbol{x}$.

For constructing graphical spectral sketches, we closely follow the approach of Jambulapati and Sidford [JS18] and Andoni et al. [ACK ${ }^{+}$16]. However, to construct sketches which are graphical, we use an approach similar to the degree-preserving sparsification algorithm. Our result is as follows:

Theorem 3.6. Given $\varepsilon \in(0,1]$, every undirected graph $G$ with $n$ vertices and $m$ edges has a graphical $\varepsilon$-spectral sketch of $G$ with $\widetilde{O}\left(n \varepsilon^{-1}\right)$ edges. The algorithm SPECTRALSKETCH, given $G$, runs in time $m^{1+o(1)}$, and with high probability returns a graphical $\varepsilon$-spectral sketch of $G$ with $n^{1+o(1)} \varepsilon^{-1}$ edges. In addition, both these graphical sketches satisfy $y^{4}$ that for any fixed $\boldsymbol{x} \in \mathbb{R}^{n}$, with high probability over the sample $H \sim \mathcal{H}$, we have $\boldsymbol{x}^{\top} \boldsymbol{L}_{G}^{+} \boldsymbol{x} \approx_{\varepsilon} \boldsymbol{x}^{\top} \boldsymbol{L}_{H}^{+} \boldsymbol{x}$.

The key idea in [JS18] and [ACK $\left.{ }^{+} 16\right]$ is to focus on an expander $G$, and for each vertex $u$ with degree $d_{u}$, sample $\varepsilon^{-1}$ edges incident at $u$ and add them to $H$ after scaling its weight by $d_{u} \varepsilon$ (if $d_{u} \leq \varepsilon^{-1}$, we add all the edges of $u$ to $H$ ), for a total of $n \varepsilon^{-1}$ edges. Firstly, observe that this means that we will have vertices where the degree changes by $\sqrt{\varepsilon^{-1}}$. This is not good enough to preserve $\boldsymbol{x}^{\top} \boldsymbol{L}_{G} \boldsymbol{x}$ up to $(1 \pm \varepsilon)$ even for the vectors $\boldsymbol{x} \in\left\{\boldsymbol{\chi}_{u}\right\}_{u \in V}$. They get around this by explicitly storing the diagonal degree matrix $\boldsymbol{D}_{G}$ of $G$ using $O(n)$ extra space. For a fixed vector $\boldsymbol{x}$, they consider the estimator $\boldsymbol{x}^{\top} \boldsymbol{D}_{G} \boldsymbol{x}-\boldsymbol{x}^{\top} \boldsymbol{A}_{H} \boldsymbol{x}$. Its expectation is easily seen to be $\boldsymbol{x}^{\top} \boldsymbol{L}_{G} \boldsymbol{x}$. They prove that its standard deviation is bounded by $\varepsilon \cdot O\left(\boldsymbol{x}^{\top} \boldsymbol{D}_{G} \boldsymbol{x}\right)$. For an expander with conductance $\phi$, Cheeger's inequality (see Lemma 6.5) gives that $\varepsilon \cdot \boldsymbol{x}^{\top} \boldsymbol{D}_{G} \boldsymbol{x}=\varepsilon \cdot O\left(\phi^{-2} \boldsymbol{x}^{\top} \boldsymbol{L}_{G} \boldsymbol{x}\right)$.

In order to construct an estimator for general graphs, they invoke expander partitioning $[\mathrm{ST} 11 \mathrm{~b}$ ], which guarantees that in any graph $G$, we can find disjoint vertex induced pieces $G$, such that each piece is contained in an expander (a well-connected subgraph, formally defined in Section 6.2), and at least half the edges are contained within such pieces. Applying this $O(\log n)$ times recursively, and combining the above estimators for each piece, Jambulapati and Sidford JS18] obtain an estimator with standard deviation $\varepsilon \cdot \widetilde{O}\left(\boldsymbol{x}^{\top} \boldsymbol{L}_{G} \boldsymbol{x}\right)$.

The above sketch is not a graph since sampling edges does not preserve degrees exactly. Hence, our degree-preserving sparsification algorithm presents a natural approach to convert it into a graphical sketch. We aim to reduce the edge count by a constant factor without incurring too much

\footnotetext{
${ }^{4} \boldsymbol{L}^{+}$denotes the Moore-Penrose pseudo-inverse of $\boldsymbol{L}$.
} 
variance in the quadratic form (and then repeat this process $O(\log n)$ times). We apply expander decomposition, and within each piece, add all edges incident on vertices with degree at most $\widetilde{O}\left(\varepsilon^{-1} L\right)$ to $H$. On the remaining graph, as before, we find a bi-partition, a cycle decomposition, and independently pick odd/even edges in the cycles with double the weight. This reduces the number of edges by a constant factor. Since we preserve weighted degrees exactly, an analysis similar to the above gives that for a fixed vector $\boldsymbol{x}$ the standard deviation in $\boldsymbol{x}^{\top} \boldsymbol{L}_{H} \boldsymbol{x}$ is bounded by $\varepsilon \cdot \widetilde{O}\left(\boldsymbol{x}^{\top} \boldsymbol{L}_{G} \boldsymbol{x}\right)$. Repeating this process $O(\log n)$ times gives us a graph $H$ with $\widetilde{O}\left(\widehat{m}+n \varepsilon^{-1} L\right)$ edges. Averaging $\widetilde{O}(1)$ such sketches, and applying concentration bounds, we obtain a graphical $\varepsilon$-spectral sketch of $G$.

The fact that we have a graph allows us to reason about the quadratic form of its inverse $\boldsymbol{x}^{\top} \boldsymbol{L}_{H}^{+} \boldsymbol{x}$. We first argue that $H$ is an $\sqrt{\varepsilon}$-spectral sparsifier of $G$ by showing that the probabilities that we sample edges to form $H$ are good upper bounds of (appropriate rescalings of) effective resistances. This follows because any edge $e$ incident to vertices with degrees at least $\varepsilon^{-1}$ that are contained in an expander with expansion at least $\phi$ has effective resistance at most $O\left(\phi^{-2} \varepsilon\right)$.

A simple, but somewhat surprising argument (Lemma 6.8) gives that if $H$ is a graphical $\varepsilon$ spectral sketch, and a $\sqrt{\varepsilon}$-spectral sparsifier, then for any fixed vector $x$, with high probability, it also preserves the inverse quadratic form of $G$, i.e., $\boldsymbol{x}^{\top} \boldsymbol{L}_{H}^{+} \boldsymbol{x} \approx_{O(\varepsilon)} \boldsymbol{x}^{\top} \boldsymbol{L}_{G}^{+} \boldsymbol{x}$.

Picking $\boldsymbol{x} \in\left\{\chi_{u v} \mid u, v \in V\right\}$, and taking union bound, we obtain that with high probability, for all $u, v \in V, R_{\mathrm{eff}}^{G}(u, v) \approx_{O(\varepsilon)} R_{\mathrm{eff}}^{H}(u, v)$. This means that $H$ is a resistance-sparsifier for $G$ with high probability. Again, the naive cycle decomposition gives the existence of resistance-sparsifiers with $\widetilde{O}\left(n \varepsilon^{-1}\right)$ edges.

Corollary 3.7 (Resistance Sparsifiers). For $\varepsilon \in(0,1]$, every undirected graph $G$ on $n$ vertices has a resistance sparsifier with $\widetilde{O}\left(n \varepsilon^{-1}\right)$ edges. The algorithm SPECTRALSKETCH, given $G$, runs in time $m^{1+o(1)}$, and with high probability returns a resistance sparsifier of $G$ with $n^{1+o(1)} \varepsilon^{-1}$ edges.

\subsection{Estimating Effective-Resistances}

The effective resistance of an edge is a fundamental quantity. It and its extensions have a variety of connections in the analysis of networks SM07, Sar10], combinatorics [Lov93, DFGX18] and the design of better graph algorithms [CKM ${ }^{+} 11$, MST15, Sch17].

While the effective resistance of an edge $u v$ can be computed to high accuracy using linear system solvers, doing so for all edges leads to a quadratic running time. On the other hand, the many algorithmic applications of resistances have motivated studies on efficient algorithms for estimating all resistances. There have been two main approaches for estimating effective resistances to date: random projections [SS11, KLP12] or recursive invocations of sparsified Gaussian elimination $\left.\mathrm{DKP}^{+} 17\right]$. Both of them lead to running times of $\widetilde{O}\left(m \varepsilon^{-2}\right)$ for producing $1 \pm \varepsilon$ estimates of the resistances of all $m$ edges of a graph.

A recent result by Musco et al. [MNS ${ }^{+}$] ] demonstrated the unlikelihood of high accuracy algorithms (with $\varepsilon^{-c}$ dependency for some small $c$ ) for estimating the resistances of all edges. On the other hand, the running time of a determinant estimation algorithm for Laplacians by Durfee et al. [DPPR17] hinges on this $\varepsilon$ dependency. The running time bottleneck of this algorithm is the estimation of effective resistances of $\widetilde{O}\left(n^{1.5}\right)$ edges, but to an multiplicative error of $\varepsilon=n^{-0.25}$. Both methods for estimating resistances described above [SS11, $\mathrm{DKP}^{+} 17$ ] give running times of $\widetilde{O}\left(n^{2}\right)$ in this setting. Practical studies involving the random projection method for estimating resistances [Sar10, MGKT15] also demonstrate the $\log n \varepsilon^{-2}$ factor in the runtime of such methods 
translates to solving $10^{3}$ linear systems for a $10 \%$ error. Such high overhead has been a major limitation in applying effective resistances to analyzing networks.

A key advantage of our graph sketches and resistance sparsifiers is that because the resulting objects remain as graphs, they can be substituted into the intermediate states of the sparsified Gaussian elimination approach for computing graph sparsifiers [ $\mathrm{DKP}^{+} 17$ ]. They give a reduction from computing effective resistances to computing approximate Schur complements, which are partial states of Gaussian elimination. Incorporating our spectral sketches in place of generic graph sparsification algorithms with $\varepsilon^{-2}$ dependencies gives our main algorithmic result.

Theorem 3.8. Given any undirected graph $G$ with $m$ vertices, and $n$ edges, and any $t$ vertex pairs and error $\varepsilon>0$, we can with high probability compute $\varepsilon$-approximations to the effective resistances between all $t$ of these pairs in $O\left(m^{1+o(1)}+(n+t) n^{o(1)} \varepsilon^{-1.5}\right)$ time.

This is the first routine for estimating effective resistances on sparse graphs that obtain an $\varepsilon$ dependence better than $\varepsilon^{-2}$. In the dense case an $\widetilde{O}\left(n^{2} \varepsilon^{-1}\right)$ result was shown by Jambulapati and Sidford [JS18], but it relies on $\Omega(n)$ linear systems solves, one per column of the matrix.

We obtain this result via two key reductions:

1. The recursive approximate Gaussian elimination approach from [ $\left.\mathrm{DKP}^{+} 17\right]$ utilizes the fact that effective resistances are preserved under Gaussian eliminations. As this recursion has depth $O(\log n)$, our guarantees for $\varepsilon$-spectral sketches imply that it suffices to work with sketches of such graphs produced by Gaussian elimination. However, Schur complement of very sparse graphs such as the degree $n$ star may have $\Omega\left(n^{2}\right)$ edges. Even if we eliminate an independent set of size $\Theta(n)$, each with roughly average degrees in our spectral sketches with $n \varepsilon^{-1}$ edges, we will end up with at least $n \varepsilon^{-2}$ edges. Thus, we need to directly compute spectral sketches of Schur complements without first constructing the dense graph explicitly.

2. The work of Kyng et al. $\mathrm{KLP}^{+} 16$ ] builds fast solvers for Laplacian systems via approximate Cholesky factorization. As a key step, they reduce computing approximating Schur complements to implicitly sparsifying a sum of product weighted cliques 5 . Assuming we start with a spectral-sketch, we know that the graph has total degree $n^{o(1)} \varepsilon^{-1}$, this implies that the total number of vertices involves in these product weighted cliques is $\varepsilon^{-1} n$. Thus, our goal becomes designing an algorithm for implicitly building spectral sketches of product-weighted cliques with a total of $n^{1+o(1)} \varepsilon^{-1}$ vertices that run in time $n^{1+o(1)} \varepsilon^{-(2-c)}$ for some constant $c>0$.

Our algorithm works with these weighted cliques in time dependent on their representation, which is the total number of vertices, rather than the number of edges. We do so by working with bi-cliques as the basic unit, instead of edges. Our algorithm then follows the expander-partitioning based scheme for producing spectral sketches, as in previous works on graph sketches with $\varepsilon^{-1}$ type dependencies [ACK ${ }^{+} 16$, JS18]. This requires showing that this representation as bi-cliques interacts well with both weights and graph partitions. Then on each well-connected piece, we sample $n^{o(1)} \varepsilon^{-0.5}$ matchings from each bi-clique.

This results in each vertex in the bi-clique representation contributing $n^{o(1)} \varepsilon^{-0.5}$ edges to the intermediate sketch. As we are running such routines on the output of spectral sketches, the total number of vertices in these cliques is $n^{o(1)} \varepsilon^{-1}$, giving a total edge count of $n^{1+o(1)} \varepsilon^{-1.5}$. On this graph, we can now explicitly compute another spectral sketch of size $n^{1+o(1)} \varepsilon^{-1}$.

\footnotetext{
${ }^{5}$ A product weighted clique has a weight vector $\boldsymbol{w}$ with the $u, v$ edge having weight $\boldsymbol{w}_{u} \boldsymbol{w}_{v}$.
} 
An additional complication is computing an expander decomposition using Lemma 6.7 requires examining all the edges of a graph, which in our case is cost-prohibitive. We resolve this by computing these decompositions on a constant error sparse approximation of this graph instead.

Incorporating this spectral sketch of Schur complements back into [DPPR17] gives the first sub-quadratic time algorithm for estimating the determinants of a graph Laplacian with the last row and column removed. This value has a variety of natural interpretations including the number of random spanning trees in the graph. Note that while the determinant may be exponentially large, the result in [DPPR17] is stable with variable-precision floating point numbers.

Corollary 3.9. Given any graph Laplacian $\boldsymbol{L}$ on $n$ vertices and $m$ edges, and any error $0<\varepsilon<1 / 2$, we can produce an $1 \pm \varepsilon$ estimate to $\operatorname{det}\left(\boldsymbol{L}_{-n}\right)$, the determinant of $\boldsymbol{L}$ with the last row/column removed, in time $m^{1+o(1)}+n^{15 / 8+o(1)} \varepsilon^{-7 / 4}$.

Note that the removal of the last row / column is necessary and standard due to $\boldsymbol{L}$ being low rank. Details on this algorithm, and the specific connections with [DPPR17] are in Appendix B, We remark that this algorithm however does not speed up the random spanning tree generation portion of DPPR17] due to it relying on finer variance bounds that require sampling $\widetilde{O}\left(n^{2}\right)$ edges. That spanning tree sampling algorithm however, is superseded by the recent breakthrough result by Schild [Sch17].

\subsection{Almost-Linear Time Short Cycle Decomposition}

The bottleneck in the performances of all algorithms outlined above is the computation of short cycle decompositions (Definition 1.1). The simple existence proof from Section 1.1 can be implemented to find a short cycle decomposition in $O(m n)$ time (see Section 8 for pseudo-code and proof).

Theorem 3.10. The algorithm NAIVECyCleDeComposition, given an undirected unweighted graph $G$, returns a $(2 n, 2 \log n)$-short cycle decomposition of $G$ in $O(m n)$ time.

While the above algorithm gives us near-optimal length and number of remaining edge: 6 , we were unable to obtain an almost-linear time algorithm using shortest-path trees. The main obstacle is that updating shortest-path trees is expensive under edge deletions.

Possible Approaches via Spanners. Another approach is to try spanners. The existence of a short cycle decomposition is a direct consequence of spanners. A key result by Awerbuch and Peleg [AP90] for spanners states that every unweighted graph $G$ has a subgraph $H$ with $O(n)$ edges such that for every edge in $H \backslash G$, its end points are within distance $O(\log n)$ in $H$. Thus, every edge in $H \backslash G$ is in a cycle of length $O(\log n)$. We can remove this cycle and repeat.

Thus, another approach for generating this decomposition is by dynamic, or even decremental, spanners [BS08, BR11, BKS12]. While these data structures allow for poly $(\log n)$ time per update, they are randomized, and crucially, only work against oblivious adversaries. Thus, the update sequence needs to fixed before the data structure samples its randomness. To the best of our understanding, in each of these result, the choice of cycle edges depends upon the randomness. Thus, their guarantees cannot be used for constructing short cycle decompositions. The only deterministic

\footnotetext{
${ }^{6}$ Consider the wheel graph with $\frac{n-1}{\log n}$ spokes, and replace each spoke with a path of length $\log n$. This graph has $n$ vertices, $(n-1)\left(1+\frac{1}{\log n}\right)$ edges, and girth of $2 \log n+1$. Lubotzky, Philip, and Sarnak [LPS88] constructed explicit Ramanujan graphs that are 4-regular (and hence have $2 \mathrm{n}$ edges) and girth $\frac{4}{3} \log _{3} n \geq 0.84 \log _{2} n$.
} 
dynamic spanner algorithm we're aware of is the work of Bodwin and Krinninger [BK16]. However, it has overheads of at least $\sqrt{n}$ in the spanner size / running time.

Possible Approaches via Oblivious Routings. Another possible approach of finding short cycles is via oblivious routings: a routing of an edge $e$ (that doesn't use $e$ ) immediately gives a cycle involving $e$. Since there exist oblivious routings for routing all edges of $G$ in $G$ with small congestion, the average length of a cycle cannot be too large.

Recent works, especially those related to approximate maximum flow, have given several efficient constructions of oblivious routing schemes [Rac08, RST14, Mad10, She13, KLOS14, Pen16]. However, such routings only allow us to route a single commodity in nearly-linear time. Using current techniques, routing $\Omega(n)$ arbitrary demands on an expander with poly $(\log n)$ congestion seems to requires $n^{1.5}$ time. On the other hand, on more limited topologies, it is known how to route each demand in sub-linear time [Val82]. Such a requirement of only using local information to route have been studied as myopic routing [GSY17], but we are not aware of such results with provable guarantees.

Our Construction. As an important technical part of this paper, we give an almost-linear-time algorithm for constructing a short cycle decomposition of a graph.

Theorem 3.11. The algorithm ShorTCyCLEDECOMPOSITIOn, given an undirected unweighted graph $G$ with $n$ vertices and $m$ edges, returns a $\left(n^{1+o(1)}, n^{o(1)}\right)$-short cycle decomposition of $G$ in $m^{1+o(1)}$ time.

Our construction of short cycle decomposition is inspired by oblivious routings, and uses the properties of random walks on expanders. This can be viewed as extending previous works that utilize behaviors of electrical flows [KM11, KLOS14], but we take advantage of the much more local nature of random walks. This use of random walks to port graphs to fewer vertices is in part motivated by their use in the construction of data structures for dynamically maintaining effective resistances, involving a subset of the authors [DGGP18]. It also has similarities with the leader election algorithm for connectivity on well-connected graphs in a recent independent work by Assadiet al. [ASW18].

Say we have an expander graph $G$ with conductance $\phi$. We know random walks of length $\phi^{-2} \log n$ mix well in $G$. Choose a parameter $k$ say $n^{1 / 10}$, and pick the set $S$ of $n / k$ vertices of largest degree (with total degree at least $2 \mathrm{~m} / \mathrm{k}$ ). For every edge $e$ leaving $S$, starting from its other end point $u \notin S$, we take a $O\left(\phi^{-2} \log n\right)$ step random walk. This random walk hits $S$ again with probability $\Omega(1 / k)$. Thus, if we pick $k \log n$ random walks, at least one of them will hit $S$ again with high probability. This is a short cycle in $G_{/ S}(G$ with $S$ contracted to a single vertex). Since these are independent random walks, Chernoff bounds imply that the maximum congestion is $\widetilde{O}\left(k \phi^{-2}\right)$. Thus, we can greedily pick a set of $\widetilde{\Omega}\left(m \phi^{4} k^{-1}\right)$ cycles of length $\widetilde{O}\left(\phi^{-2}\right)$ in $G_{/ S}$ that are disjoint.

Now, we just need to connect these cycles within $S$. We define a graph on the vertices of $S$, with one edge for every cycle in $G_{/ S}$ connecting the two end points in $S$, and recurse on $S$. With 10 levels of recursion ( ince $k=n^{1 / 10}$ ), and using the naive cycle-decomposition for the base case, we find a short cycle decomposition in this graph, and then can expand it to a cycle decomposition in $G$ using the cycles in $G_{/ S}$. This should give cycles of length $\left(\phi^{-2} \log n\right)^{10}$.

There is a key obstacle here: this approach really needs expanders, not pieces contained in expanders, as in the expander decomposition from Spielman and Teng [ST11a]. Instead, we use a recent result of Nanongkai and Saranurak [NS17] that guarantees the pieces are expanders, at 
the cost of achieving $\phi=n^{-o(1)}$, and a running time of $m^{1+o(1)}$. A careful trade-off of parameters allows us to recurse for $n^{o(1)}$ iterations, resulting in an $\left(n^{1+o(1)}, n^{o(1)}\right)$-short cycle decomposition in $m^{1+o(1)}$ time.

\section{Cycle Decomposition algorithm in the following sections}

In the following sections, we assume CyCLEDECOMPosition is an algorithm that takes as input an unweighted graph with $n$ vertices and $m$ edges, runs in time at most $\mathrm{T}_{\text {CyCleDecomp }}(m, n) \geq m$, and returns a $(\widehat{m}(n), L(n))$-short cycle decomposition. Further, we assume that $T$ satisfies

$$
\sum_{i} \mathrm{~T}_{\text {CycleDecomp }}\left(m_{i}, n\right) \leq \mathrm{T}_{\text {CycleDecomp }}\left(\sum_{i} m_{i}, n\right)
$$

for all $m_{i} \geq n$. We also assume $\mathrm{T}_{\text {Cycledecomp }}(m, n) \leq \mathrm{T}_{\text {CycleDecomp }}\left(m^{\prime}, n^{\prime}\right)$, for any $m \leq m^{\prime}, n \leq$ $n^{\prime}$. Since $n$ will remain the same throughout these sections, we will simply write $\widehat{m}$ and $L$ instead of $\widehat{m}(n)$ and $L(n)$.

\section{Degree-Preserving Spectral Sparsification}

In this section, we describe an efficient algorithm for constructing degree-preserving spectral sparsifiers, proving Theorem 3.3 .

The algorithm will use a short cycle decomposition, and sparsify each cycle $C$ with the distribution

$$
\widetilde{C}:=2 \cdot \begin{cases}\text { all odd edges of } C & \text { w.p. } 1 / 2 \\ \text { all even edges of } C & \text { w.p. } 1 / 2 .\end{cases}
$$

We will bound the error in this distribution via matrix Chernoff bounds [Tro12], and recursively apply this sparsification procedure until our graph achieves low edge count.

The following theorem is the main result of this section.

Theorem 4.1. Given a graph $G$ with integer poly bounded edge weights, an error parameter $\varepsilon$, and a cycle decomposition routine CyCleDeComposition, the algorithm DegreEPRESERVINGSPARSIFY (described in Algorithm 1) returns a graph $H$ with at most $O\left(\widehat{m} \log n+n L \varepsilon^{-2} \log n\right)$ edges such that all vertices have the same weighted degrees in $G$ and $H$, and with high probability, $\boldsymbol{L}_{G} \approx_{\varepsilon} \boldsymbol{L}_{H}$.

The algorithm DEGREEPRESERVINGSPARSIFY runs in time

$$
O\left(m \log ^{2} n\right)+\mathrm{T}_{\text {CycleDecomp }}(O(m \log n), n)
$$

We first prove Theorem 3.3 by plugging in NAIVECyCleDecomposition and ShortCyCleDeCOMPOSITION into Theorem 4.1 and evaluating $T$ on those routines. It is easy to check that their runtimes satisfy assumption 2 .

Proof of Theorem 3.3. Note that DegreePreservingSparsify always returns a graph $H$ with the same weighted degrees as $G$, such that $\boldsymbol{L}_{H} \approx_{\varepsilon} \boldsymbol{L}_{G}$ with high probability. Using either NAIVECYCleDecomposition or ShortCycleDecomposition as the algorithm CycleDecomposition, we obtain the following guarantees: 
Algorithm 1 DegreePreservingSparsify $(G, \varepsilon$, CyCleDeComposition)

Input: Graph $G$ with poly bounded edge weights.

1. Decompose each edge of $G$ by its binary representation. Now edge weights of $G$ are powers of 2 , and are at most $m \log n$ in number.

2. Compute $\boldsymbol{r}$, a 1.5-approximate estimate of effective resistances in $G$.

3. While $|E(G)| \geq \Omega\left(\widehat{m} \log n+n L \epsilon^{-2} \log n\right)$ :

(a) $G \leftarrow \operatorname{SparsifyOnce}(G, \boldsymbol{r}$, CycleDecomposition) $)$.

4. Return $G$.

1. Using NaiveCycleDecomposition: DegreePreservingSparsify runs in $O(m n \log n)$ time, and returns an $H$ with $O\left(n \varepsilon^{-2} \log ^{2} n\right)$ edges.

2. Using ShortCycleDecomposition: DegreePreservingSparsify runs in $m^{1+o(1)}$ time, and returns an $H$ with $n^{1+o(1)} \varepsilon^{-2}$ edges.

Thus we have our theorem.

In order to prove Theorem 4.1, we first prove the following lemma about the effect of sampling the cycles independently. It is a direct consequence of matrix concentration bounds.

Lemma 4.2. Let $\mathcal{G}_{1} \ldots \mathcal{G}_{t}$ be independent distributions over graphs containing at most $L$ edges, and let their expectations be

$$
G_{i} \stackrel{\text { def }}{=} \underset{\widetilde{G}_{i} \sim \mathcal{G}_{i}}{\mathbb{E}}\left[\widetilde{G}_{i}\right]
$$

and define their sum to be $G \stackrel{\text { def }}{=} \sum_{1 \leq i \leq t} G_{i}$. For any graph $H$ with

$$
\boldsymbol{L}_{G} \preceq \boldsymbol{L}_{G}
$$

such that the maximum leverage score of any edge with respect to $H$ in bounded above by $\rho$, the random graph

$$
\widetilde{G}=\widetilde{G}_{1}+\ldots+\widetilde{G}_{t}
$$

with $\widetilde{G}_{i} \sim \mathcal{G}_{i}$ satisfies with high probability

$$
\boldsymbol{L}_{H} \approx_{O(\sqrt{L \rho \log n})} \boldsymbol{L}_{\widetilde{G}+H-G} .
$$

Proof. This is a corollary of Matrix Chernoff bounds from [Tro12], which state that for a sequence of independent random $d \times d$ PSD matrices $\left\{\boldsymbol{X}_{i}\right\}$ such that $\mathbb{E}\left[\sum_{i} \boldsymbol{X}_{i}\right] \preceq \boldsymbol{T}$, we have for $\delta \leq 1$,

$$
\mathbb{P}\left[\mathbb{E}\left[\sum \boldsymbol{X}_{i}\right]-\sum \boldsymbol{X}_{i} \preceq \delta \boldsymbol{T}\right] \geq 1-d \cdot e^{\frac{-\delta^{2}}{3 R}}
$$

and

$$
\mathbb{P}\left[\mathbb{E}\left[\sum \boldsymbol{X}_{i}\right]-\sum \boldsymbol{X}_{i} \succeq-\delta \boldsymbol{T}\right] \leq 1-d \cdot e^{\frac{-\delta^{2}}{3 R}}
$$


where $R$ is such that for each $i$, we have $R \leq\left\|\boldsymbol{T}^{+/ 2} \boldsymbol{X}_{i} \boldsymbol{T}^{+/ 2}\right\|$ almost surely.

To prove Lemma 4.2, we set $\boldsymbol{X}_{i}=\boldsymbol{L}_{\widetilde{G}_{i}}, \boldsymbol{S}=\boldsymbol{L}_{\widetilde{G}}$, and $\boldsymbol{T}=\boldsymbol{L}_{H}$. Then,

$$
\left\|\boldsymbol{T}^{+/ 2} \boldsymbol{X}_{i} \boldsymbol{T}^{+/ 2}\right\|_{2} \leq \operatorname{Tr}\left(\boldsymbol{T}^{+/ 2} \boldsymbol{X}_{i} \boldsymbol{T}^{+/ 2}\right)=\sum_{e \in \widetilde{G}_{i}} \operatorname{Tr}\left(\boldsymbol{L}_{H}^{+/ 2} \boldsymbol{L}_{e} \boldsymbol{L}_{H}^{\dagger / 2}\right) \leq \rho L,
$$

where the last inequality follows since the number of edges in $\widetilde{G}_{i}$ is at most $L$, and $\operatorname{Tr}\left(\boldsymbol{L}_{H}^{+/ 2} \boldsymbol{L}_{e} \boldsymbol{L}_{H}^{+/ 2}\right)$ is the definition of the leverage score of any edge $e$ in the support of $\mathcal{G}$ w.r.t. $H$. Note that our edge leverage scores are bounded above by $\rho$. Now, we can set $\delta=O(\sqrt{\rho L \log n})$ to get

$$
\mathbb{P}\left[\mathbb{E}\left[\sum \boldsymbol{L}_{\widetilde{G}_{i}}\right]-\boldsymbol{L}_{G} \preceq O(\sqrt{L \rho \log n}) \boldsymbol{L}_{H}\right] \geq 1-\frac{1}{n^{O(1)}}
$$

or equivalently,

$$
\mathbb{P}\left[\boldsymbol{L}_{\widetilde{G}+H-G} \preceq(1+O(\sqrt{L \rho \log n})) \boldsymbol{L}_{H}\right] \geq 1-\frac{1}{n^{O(1)}}
$$

Similarly, we bound the other direction, and by the union bound, we obtain with high probability:

$$
\exp (-O(\sqrt{L \rho \log n})) \boldsymbol{L}_{H} \preceq \boldsymbol{L}_{\tilde{G}+H-G} \preceq \exp (O(\sqrt{L \rho \log n})) \boldsymbol{L}_{H} .
$$

The correctness of algorithm DegreePreservingSparsify hinges on the following lemma about SPARSIFYONCE.

Lemma 4.3. Given a graph $G$ with edge weights that are polynomially bounded and powers of 2, a 2-approximate estimate $\boldsymbol{r}$ for effective resistances in $G$, and a cycle decomposition routine CyCleDecomposition, the algorithm SPARSIFyOncereturns a graph $H$ on the same vertex set that preserves all weighted vertex degrees, and with high probability,

$$
\boldsymbol{L}_{H} \approx_{O\left(\sqrt{\frac{n L \log n}{m}}\right)} \boldsymbol{L}_{G}
$$

Further, when $m \geq \Omega(\widehat{m} \log n)$, $H$ has at most (15/16)m edges with high probability.

Proof. First, we show that SparsifyOnCEreturns a graph that is a spectral sparsifier of $G$ with the parameters specified above. The difference between $G$ and $H$ arises from sampling the cycles $\left\{C_{i j}\right\}_{i j}$ produced by CycleDecomposition. Note that each cycle $C_{i j}$ must be even-length since its a cycle in the bipartite graph $G_{i}$.

For an even length cycle $C_{i j}$, recall the algorithm samples either twice the odd indexed edges, or twice the even indexed edges of $C_{i j}$, each with probability $1 / 2$. Let distribution $\widetilde{C}_{i j}$ denote the distribution that returns twice the even edges of $C_{i j}$ with $1 / 2$ probability and twice the odd edges with $1 / 2$ probability. Also, note that all edges in $C$ have leverage score at most $8 \mathrm{n} / \mathrm{m}$.

Note that $H$ is obtained by removing all cycles $C_{i j}$ from $G$, and then adding the sampled odd/even edges $\widetilde{C}_{i j}$ with twice the weights. Thus,

$$
H=\left(G \backslash \sum_{i j} C_{i j}\right)+\sum_{i j} \widetilde{C}_{i j}
$$




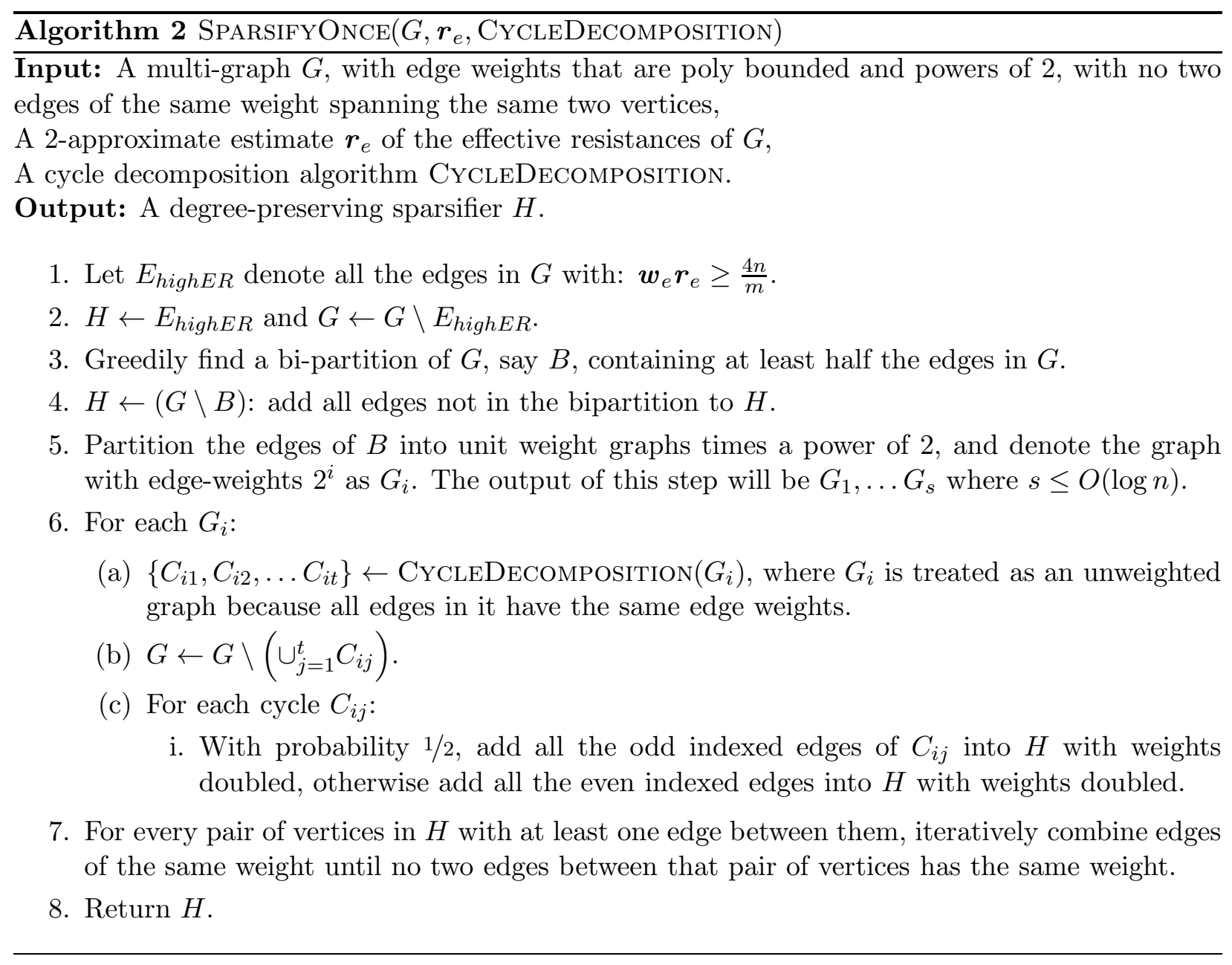


Observing that each cycle $C_{i j}$ has at most $L$ edges, along with $\mathbb{E}\left[\widetilde{C}_{i j}\right]=C_{i j}$, and we can apply the concentration bound from Lemma 4.2, to obtain

$$
\boldsymbol{L}_{H} \approx_{O\left(\sqrt{\frac{n L \log n}{m}}\right)} \boldsymbol{L}_{G}
$$

Next, we show that $H$ has at most $(15 / 16) m$ edges. The edges removed are:

1. $\leq m / 2$ edges with low leverage score.

Since $\boldsymbol{r}$ is a 2-approximate estimate of effective resistance, by Foster's theorem, the average value of $\boldsymbol{w}_{e} \boldsymbol{r}_{e}$ of all edges is at most $2(n-1) / m$. So at most half of the edges in $G$ have $\boldsymbol{w}_{e} \boldsymbol{r}_{e} \geq \frac{4 n}{m}$.

2. $\leq m / 4$ edges not in the bipartition.

At most half the high ER edges are not in the bipartition.

3. $\leq m / 8$ edges not in cycles.

At most $O(\widehat{m} \log n)$ edges are not in the cycles since $i \leq O(\log n)$. If $m=\Omega(\widehat{m} \log n)$, this number does not exceed $m / 8$. This leaves at least $m / 8$ edges in cycles.

4. We add exactly half the cycle edges to $H$.

This completes our proof.

Lemma 4.4. Given a multi-graph $G$ with $m^{\prime}$ edges satisfying the input assumptions of SPARSIFYONCE, a vector $\boldsymbol{r}$ that are 2-approximations to the effective resistances of $G$, and a cycle decomposition routine CYCleDeCOMPOSITION, the algorithm SPARSIFyOnCEruns in time at most

$$
O\left(m^{\prime}\right)+\mathrm{T}_{\text {CyCleDecomp }}\left(m^{\prime}, n\right) .
$$

Proof. It takes $O\left(\mathrm{~m}^{\prime}\right)$ time to greedily find a bipartition in Step 3, For the second term, we analyze the time for the cycle decomposition steps. The total time taken by the cycle decomposition steps is at most

$$
\sum_{i} \mathrm{~T}_{\text {CYCLEDeComp }}\left(\left|E\left(G_{i}\right)\right|, n\right) .
$$

Here $G_{i}$ are the graphs from step 5 in Algorithm [2, By our assumption on $\mathrm{T}_{\text {CycleDecomp }}$ in Equation 2, this term is bounded above by $\mathrm{T}_{\text {CycleDecomp }}\left(\sum_{i}\left|E\left(G_{i}\right)\right|, n\right)=\mathrm{T}_{\text {Cycledecomp }}\left(m^{\prime}, n\right)$.

Finally, we are ready to prove Theorem 4.1 .

Proof of Theorem 4.1. First observe that the DegreePreservingSPARSify must return a graph with $O\left(\widehat{m} \log n+n L \varepsilon^{-2} \log n\right)$ edges.

Lemma 4.3 tells us that the weighted degree of each vertex is preserved throughout the algorithm. Therefore, the graph resulting from DegreePreservingSparsify $(G, \varepsilon, \operatorname{CyCleDecomposition})$ is guaranteed to have the same weighted degrees as the original graph.

Let $H_{i}$ be the value of $G$ after the $i^{t h}$ iteration of the loop in DEgreEPRESERvingSparsify (Line 3), and let $m_{i}$ be the number of edges in $H_{i}$. Lemma 4.3 tells us that with high probability, $H_{i+1}$ is a $O\left(\sqrt{\frac{n \log n}{m_{i}}}\right)$-sparsifier of $H_{i}$, and $m_{i+1} \leq \frac{15}{16} m_{i}$. Suppose the loop runs for $t$ iterations before it terminates. Then $H_{t}$ is the final output of DegreePreservingSparsify. 
By construction, the error is bounded by

$$
\sum_{i=0}^{t-1} O\left(\sqrt{\frac{n \log n}{m_{i}}}\right)
$$

Since $m_{i}$ decreases geometrically, this can be bounded in terms of the largest term in the product, which is:

$$
O\left(\sqrt{\frac{n L \log n}{m_{t-1}}}\right) \leq \varepsilon
$$

The last inequality holds because $m_{t-1} \geq \Omega\left(n L \varepsilon^{-2} \log n\right)$. Therefore, DegreePRESERvingSparsify returns a low-error sparsifier with high probability.

Now we analyze the time bound. Note that to compute 1.5-approximate effective resistances, it takes $O\left(m \log ^{2} n\right)$ time [SS11, KLP12]. We perform this computation just once, since our cumulative error is bounded, and a 2-approximation to effective resistances suffices for us.

During each call to SPARSIFYONCEin Algorithm 1, the number of edges in the input is $m_{i}$, and the runtime of a single loop is bounded above by $\mathrm{T}_{\text {Cycledecomp }}\left(m_{i}, n\right)$ due to Lemma 4.4. Here, $m_{i}$ once again refers to $\left|E\left(H_{i}\right)\right|$. Therefore, the total runtime is at most

$$
O\left(m \log ^{2} n\right)+\sum_{i=0}^{t} \mathrm{~T}_{\text {CycleDecomp }}\left(m_{i}, n\right) .
$$

Note that $m_{0} \leq m \log n$. Since $m_{i}$ decreases more than geometrically due to Lemma 4.3, we can use Lemma 4.4 and Equation 2 to bound the second term in the sum above by

$$
O\left(m \log ^{2} n\right)+\mathrm{T}_{\text {CyCLeDecomp }}(O(m \log n), n),
$$

giving us our claimed runtime.

\section{$5 \quad$ Sparsification of Eulerian Directed Graphs}

In this section, we show how we can use short cycle decompositions to sparsify Eulerian directed graphs. For a directed graph $\vec{G}$, its directed Laplacian, $\boldsymbol{L}_{\vec{G}}$, can be defined as

$$
\boldsymbol{L}_{\vec{G}}(u, v):= \begin{cases}\text { out-degree of } u & \text { if } u=v, \\ -(\text { weight of edge } v \rightarrow u) & \text { if } u \neq v \text { and } v \rightarrow u \text { is an edge. }\end{cases}
$$

Given a directed graph $\vec{G}$, its "undirectification", $G$, is defined as the graph formed by replacing every edge $\vec{e}$ in $\vec{G}$ with an undirected edge $e$ of half the weight, with the same endpoints.

A particularly important class of directed Laplacians is the set of Laplacian matrices that correspond to Eulerian directed graphs, that is, directed graphs in which the in-degree of each vertex is the same as its out-degree. This case is shown to be complete for solving directed linear systems $\left[\mathrm{CKP}^{+} 16\right]$. Furthermore, $\vec{G}$ being Eulerian implies that its Laplacian is directly related to the Laplacian of its undirectification,

$$
\boldsymbol{L}_{G}=\frac{1}{2}\left(\boldsymbol{L}_{\vec{G}}+\boldsymbol{L}_{\vec{G}}^{\top}\right) .
$$


$\boldsymbol{L}_{G}$ is a graph Laplacian, and hence positive-semidefinite. Our main result is an efficient algorithm for sparsifying $\boldsymbol{L}_{\vec{G}}$. Because $\boldsymbol{L}_{\vec{G}}$ may be asymmetric, we will bound the deviation of our sparsifier w.r.t. the norm defined by $\boldsymbol{L}_{G}$. The pseudocode is given in Algorithm 3 ,

The sparsification algorithm will once again work with cycles. Given a cycle $\vec{C}$ with directed edges, we sparsify it with the distribution

$$
\widetilde{C}:=2 \cdot \begin{cases}\text { all clockwise edges of } \vec{C} & \text { w.p. } 1 / 2 \\ \text { all counterclockwise edges of } \vec{C} & \text { w.p. } 1 / 2\end{cases}
$$

Note, that in the case of directed graphs, we do not need our cycles to have even length, and hence can get rid of the bipartition step required for degree-preserving sparsifiers (Section 4). We will bound the error caused by this sampling procedure via rectangular matrix Chernoff bounds [Tro12]. It also appears as Theorem A.1. in Appendix A of [ $\left.\mathrm{CKP}^{+} 17\right]$.

Algorithm 3 EulerianSparsify $(\vec{G}, \epsilon$, CycleDecomposition)

Input: Eulerian directed graph $\vec{G}$ with integer, polynomially bounded edge weights.

1. Decompose each edge of $\vec{G}$ by its binary representation. Now edge weights of $\vec{G}$ are powers of 2 , and are at most $m \log n$ in number.

2. Compute $\boldsymbol{r}$, a 1.5-approximate estimate of effective resistances in $G$.

3. While $|E(\vec{G})| \geq 8 \widehat{m} \log n+O\left(n L^{3} \epsilon^{-2} \log n\right)$

(a) $\vec{G} \leftarrow$ DirectedSparsifyOnce $(\vec{G}, \boldsymbol{r}$, CycleDecomposition $)$.

4. Return $\vec{G}$.

Theorem 5.1. Given an Eulerian directed graph $\vec{G}$ with poly bounded edge weights 7 , and a cycle decomposition routine CYCLEDECOMPOSITION, the algorithm EULERIANSPARSIFY returns an Eulerian directed graph $\vec{H}$ with at most $O\left(\widehat{m} \log n+n L^{3} \epsilon^{-2} \log n\right)$ edges such that with high probability,

$$
\left\|\boldsymbol{L}_{G}^{+/ 2}\left(\boldsymbol{L}_{\vec{G}}-\boldsymbol{L}_{\vec{H}}\right) \boldsymbol{L}_{G}^{+/ 2}\right\|_{2} \leq \epsilon
$$

The algorithm EULERIANSPARSIFYruns in time

$$
O\left(m \log ^{2} n\right)+\mathrm{T}_{\text {CyCleDecomp }}(O(m \log n), n),
$$

and its pseudocode is in Algorithm 3 .

Plugging in the guarantees of NAiveCycleDecomposition from Theorem 3.10 then gives the existence of smaller sparsifiers for Eulerian Laplacians as stated in Theorem 3.4. Note that in Theorem 5.1 we assume the edge weights are integers. To deal with general weights, we only need to split each edge by the binary representation of its weight and ignore insignificant bits. Details are described in Appendix $\mathrm{A}$.

\footnotetext{
${ }^{7}$ We also assume that $\vec{G}$ has no parallel edges.
} 
Lemma 5.2. (Theorem 1.6 from [Tro12]) Let $\boldsymbol{X}_{1}, \boldsymbol{X}_{2}, \ldots, \boldsymbol{X}_{k}$ be $n \times n$ random matrices such that

1. they are 0 -matrices in expectation

$$
\mathbb{E}\left[\boldsymbol{X}_{i}\right]=0
$$

2. they have small norm:

$$
\left\|\boldsymbol{X}_{i}\right\|_{2} \leq O\left(\sqrt{\frac{n L^{3}}{m \log n}}\right)
$$

3. their expected outer and inner products are small (in summation),

$$
\left\|\sum_{1 \leq i \leq k} \mathbb{E}\left[\boldsymbol{X}_{i} \boldsymbol{X}_{i}^{\top}\right]\right\|_{2},\left\|\sum_{1 \leq i \leq k} \mathbb{E}\left[\boldsymbol{X}_{i}^{\top} \boldsymbol{X}_{i}\right]\right\|_{2} \leq O\left(\frac{n L^{3}}{m}\right) .
$$

Then w.h.p., the 2-norm of the sum is small:

$$
\mathbb{P}\left[\left\|\sum_{1 \leq i \leq k} \boldsymbol{X}_{i}\right\|_{2}>\sqrt{\frac{n L^{3} \log n}{m}}\right] \leq n^{-\Omega(1)} .
$$

Specifically, this can be obtained from Theorem 1.6 of [Tro12] with the parameters

$$
\begin{array}{rlrl}
d_{1} & =d_{2}=n, & t & =\sqrt{\frac{n L^{3} \log n}{m}} \\
R & =O\left(\sqrt{\frac{n L^{3}}{m \log n}}\right), & \sigma^{2}=O\left(\frac{n L^{3}}{m}\right) .
\end{array}
$$

This motivates us to give such bounds for each cycle whose total effective resistance is small. This will be by decomposing the terms, for which we need the following lemma. It is a direct consequence of the Cauchy-Schwarz inequality.

Lemma 5.3. Let $\boldsymbol{A}_{1} \ldots \boldsymbol{A}_{\ell}$ be matrices with sum $\boldsymbol{A}:=\sum_{i=1}^{\ell} \boldsymbol{A}_{i}$, then we have

$$
\boldsymbol{A} \boldsymbol{A}^{\top} \preceq \ell \cdot \sum_{i} \boldsymbol{A}_{i} \boldsymbol{A}_{i}^{\top}
$$

Proof. By the Cauchy-Schwarz inequality, for any set of values $a_{1} \ldots a_{\ell}$, we have

$$
\left(\sum_{i=1}^{\ell} a_{i}\right)^{2} \leq\left(\sum_{i=1}^{\ell} a_{i}^{2}\right)\left(\sum_{i=1}^{\ell} 1\right)
$$

Applying this entry-wise to vectors $\boldsymbol{y}_{1} \ldots \boldsymbol{y}_{\ell}$ gives

$$
\left\|\sum_{i=1}^{\ell} \boldsymbol{y}_{i}\right\|_{2}^{2} \leq \ell \cdot \sum_{i=1}^{\ell}\left\|\boldsymbol{y}_{i}\right\|_{2}^{2}
$$


Then substituting in $\boldsymbol{y}_{i} \leftarrow \boldsymbol{A} \boldsymbol{x}_{i}$ gives

$$
\left\|\sum_{i=1}^{\ell} \boldsymbol{A}_{i} \boldsymbol{x}\right\|_{2}^{2} \leq \ell \cdot \sum_{i=1}^{\ell}\left\|\boldsymbol{A}_{i} \boldsymbol{x}\right\|_{2}^{2}
$$

or in matrix form:

$$
\boldsymbol{x}^{\top} \boldsymbol{A}^{\top} \boldsymbol{A} \boldsymbol{x} \leq \ell \cdot \sum_{i} \boldsymbol{x}^{\top} \boldsymbol{A}_{i}^{\top} \boldsymbol{A}_{i} \boldsymbol{x} .
$$

As this holds for any $\boldsymbol{x}$, we get the desired condition on the matrices.

Our key statement is as follows:

Lemma 5.4. Let $\vec{C}$ be a equal weighted directed cycle of length $L$ contained in a graph $\vec{G}$ where each edge $\vec{e} \in \vec{C}$ satisfies

$$
E R_{G}(e) \leq \rho / \boldsymbol{w}_{e}
$$

where $\boldsymbol{w}_{e}$ is the weight of e (and all edges in $\vec{C}$ ). Let $C$ be the undirectification of $\vec{C}$, and let $\vec{C}^{\mathrm{cW}}$ represent only the clockwise edges in $\vec{C}$. Let $\widetilde{\boldsymbol{L}}$ be $2 \boldsymbol{L}_{\vec{C}^{\mathrm{cw}}}-\boldsymbol{L}_{\vec{C}}$. Then

$$
\boldsymbol{L}_{G}^{+/ 2} \cdot\left(\widetilde{\boldsymbol{L}}^{\top} \boldsymbol{L}_{G}^{+} \widetilde{\boldsymbol{L}}\right) \cdot \boldsymbol{L}_{G}^{+/ 2} \preceq O\left(L^{3} \rho\right) \cdot \boldsymbol{L}_{G}^{+/ 2} \boldsymbol{L}_{C} \boldsymbol{L}_{G}^{+/ 2}
$$

and

$$
\boldsymbol{L}_{G}^{+/ 2} \cdot\left(\widetilde{\boldsymbol{L}} \boldsymbol{L}_{G}^{+} \widetilde{\boldsymbol{L}}^{\top}\right) \cdot \boldsymbol{L}_{G}^{+/ 2} \preceq O\left(L^{3} \rho\right) \cdot \boldsymbol{L}_{G}^{+/ 2} \boldsymbol{L}_{C} \boldsymbol{L}_{G}^{+/ 2}
$$

Before proving Lemma 5.4, we will define some useful notation and state a couple of auxiliary lemmas.

For a directed edge $\vec{e} \in \vec{G}$, we denote by $\operatorname{rev}(\vec{e})$ its reversed version. Also, recall that its undirected counterpart in $G$ (with half the weight) is denoted by $e$. We also extend this notation to sets of edges.

We first reduce things to a directed cycle with only clockwise edges. Note that the directed Laplacian elements corresponding to an edge $\vec{e}$ in both directions sum up to twice the undirected Laplacian for $e$.

$$
\boldsymbol{L}_{\vec{e}}+\boldsymbol{L}_{r e v(\vec{e})}=\boldsymbol{w}_{e}\left[\begin{array}{cc}
0 & -1 \\
0 & 1
\end{array}\right]+\boldsymbol{w}_{e}\left[\begin{array}{cc}
1 & 0 \\
-1 & 0
\end{array}\right]=\boldsymbol{w}_{e}\left[\begin{array}{cc}
1 & -1 \\
-1 & 1
\end{array}\right]=2 \boldsymbol{L}_{e} .
$$

Using this in the definition of $\widetilde{\boldsymbol{L}}$ gives

$$
\widetilde{\boldsymbol{L}}=2 \boldsymbol{L}_{\vec{C}^{\mathrm{CW}}}-\boldsymbol{L}_{\vec{C}}=\boldsymbol{L}_{\vec{F}}-2 \boldsymbol{L}_{S}
$$

where $\vec{F}$ is the version of $\vec{C}$ where every edge is clockwise, and $S$ is the set of undirected edges in $G$ corresponding to counterclockwise edges in $\vec{C}$.

$$
\begin{aligned}
\boldsymbol{L}_{\vec{F}} & =\boldsymbol{L}_{\vec{C}^{\mathrm{CW}}}+\boldsymbol{L}_{r e v\left(\vec{C} \backslash \vec{C}^{\mathrm{CW}}\right)}, \\
\boldsymbol{L}_{S} & =\sum_{\vec{e} \in \vec{C} \backslash \vec{C}^{\mathrm{CW}}} \boldsymbol{L}_{e} .
\end{aligned}
$$


The triangle inequality from Lemma $\lcm{5.3}$ implies that it suffices to bound these two terms:

$$
\boldsymbol{L}_{G}^{+/ 2} \cdot\left(\boldsymbol{L}_{S} \boldsymbol{L}_{G}^{+} \boldsymbol{L}_{S}\right) \cdot \boldsymbol{L}_{G}^{+/ 2}
$$

and

$$
\boldsymbol{L}_{G}^{+/ 2} \cdot\left(\boldsymbol{L}_{\vec{C}^{\mathrm{CW}}}^{\top} \boldsymbol{L}_{G}^{+} \boldsymbol{L}_{\vec{C}^{\mathrm{CW}}}\right) \cdot \boldsymbol{L}_{G}^{+/ 2}
$$

We use the following two lemmas to derive the bounds:

Lemma 5.5. Let $S$ be the set of undirected edges in $G$ corresponding to counterclockwise edges in a cycle $\vec{C}$ in $\vec{G}$ of length at most $L$, where the effective resistances of edges in $C$ are at most $\rho$. Then,

$$
\boldsymbol{L}_{S} \preceq L \cdot \rho \cdot \boldsymbol{L}_{G} .
$$

Proof.

$$
\boldsymbol{L}_{S}=\sum_{\vec{e} \in \vec{C} \backslash \vec{C}^{\mathrm{CW}}} \boldsymbol{L}_{e}
$$

For every edge $\vec{e} \in \vec{C}$, we know that $E R_{G}(e) \leq \rho$. This, along with the definition of effective resistance in $G$, we get

$$
\boldsymbol{L}_{e} \preceq \rho \cdot \boldsymbol{L}_{G} .
$$

Using the above equations and bounding the length of $\vec{C}$ by $L$, we get the desired bound.

Lemma 5.6. Let $\vec{F}$ the graph that's a length $L$ cycle (with unit weights) such that the effective resistance between adjacent vertices in $\vec{F}$ in $G$, the undirectification of $\vec{G}$ is at most $\rho$. Then

$$
\boldsymbol{L}_{\vec{F}}^{\top} \boldsymbol{L}_{G}^{+} \boldsymbol{L}_{\vec{F}} \preceq O\left(L^{3} \cdot \rho\right) \boldsymbol{L}_{C} .
$$

Proof. Let $F$ be the undirectification of $\vec{F}$. Explicit computations of the entries of $\boldsymbol{L}_{F}^{+}$gives

$$
\boldsymbol{L}_{\vec{F}}^{\top} \boldsymbol{L}_{F}^{+} \boldsymbol{L}_{\vec{F}}=\frac{2}{L} \boldsymbol{L}_{K(L)}
$$

where $K(L)$ is a unit weighted clique on $L$ vertices. By Cauchy-Schwarz inequality,

$$
\sum_{i<j}\left(x_{i}-x_{j}\right)^{2} \leq \sum_{i<j}\left((j-i) \sum_{k=i+1}^{j}\left(x_{k-1}-x_{k}\right)^{2}\right)=O\left(L^{3}\right) \sum_{k=1}^{L}\left(x_{k-1}-x_{k}\right)^{2}
$$

where $x_{0}$ references to $x_{L}$. Thus

$$
\boldsymbol{L}_{\vec{F}}^{\top} \boldsymbol{L}_{C}^{+} \boldsymbol{L}_{\vec{F}}=\frac{2}{L} \boldsymbol{L}_{K_{L}} \preceq O\left(L^{2}\right) \boldsymbol{L}_{C} .
$$

Similar to the proof for Lemma 5.5 , by the bounds on effective resistances, we have

$$
\boldsymbol{L}_{C}=\sum_{e \in C} \boldsymbol{L}_{e} \preceq \sum_{e \in F} \rho \cdot \boldsymbol{L}_{G}=L \cdot \rho \cdot \boldsymbol{L}_{G} .
$$

Thus, $\boldsymbol{L}_{G}^{+} \preceq L \cdot \rho \cdot \boldsymbol{L}_{C}^{+}$. Substituting for $\boldsymbol{L}_{C}^{+}$gives us the claimed result. 
We are now equipped to prove Lemma 5.4 .

Proof of Lemma 5.4. Rearranging the terms from Lemma 5.5, we get

$$
\boldsymbol{L}_{S}^{1 / 2} \boldsymbol{L}_{G}^{+} \boldsymbol{L}_{S}^{1 / 2} \preceq L \cdot \rho \cdot \boldsymbol{I} .
$$

Multiplying on the left and right by the appropriate terms, and using the fact that $\boldsymbol{L}_{S} \preceq \boldsymbol{L}_{C}$, we can get

$$
\boldsymbol{L}_{G}^{+/ 2} \cdot\left(\boldsymbol{L}_{S} \boldsymbol{L}_{G}^{+} \boldsymbol{L}_{S}\right) \cdot \boldsymbol{L}_{G}^{+/ 2} \preceq L \cdot \rho \cdot \boldsymbol{L}_{G}^{+/ 2} \boldsymbol{L}_{C} \boldsymbol{L}_{G}^{+/ 2}
$$

From Lemma 5.6.

$$
\boldsymbol{L}_{G}^{+/ 2} \cdot\left(\boldsymbol{L}_{\vec{F}}^{\top} \boldsymbol{L}_{G}^{+} \boldsymbol{L}_{\vec{F}}\right) \cdot \boldsymbol{L}_{G}^{+/ 2} \preceq O\left(L^{3} \cdot \rho\right) \boldsymbol{L}_{G}^{+/ 2} \boldsymbol{L}_{C} \boldsymbol{L}_{G}^{+/ 2} .
$$

Combining these two bounds by Lemma 5.3 gives

$$
\boldsymbol{L}_{G}^{+/ 2} \cdot\left(\widetilde{\boldsymbol{L}}^{\top} \boldsymbol{L}_{G}^{+} \widetilde{\boldsymbol{L}}\right) \cdot \boldsymbol{L}_{G}^{+/ 2} \preceq O\left(L^{3} \cdot \rho\right) \cdot \boldsymbol{L}_{G}^{+/ 2} \boldsymbol{L}_{C} \boldsymbol{L}_{G}^{+/ 2}
$$

The second inequality with the transpose on the other side also follows similarly.

Algorithm 4 DirectedSparsifyOnce $(\vec{G}, \boldsymbol{r}$, CycleDecomposition $)$

Input: Eulerian directed graph $\vec{G}$, where the edge weights are integral powers of 2 ,

$r$ : 2-approximate estimates of effective resistances in $G$,

CycleDecomposition: short cycle decomposition routine.

Output: Eulerian sparsifier $\vec{H}$.

1. Construct a set $\vec{E}_{h i g h E R}$ with every edge $e \in \vec{G}$ that satisfies $\boldsymbol{w}_{e} \boldsymbol{r}_{e} \geq 4 n / m$.

2. $\vec{H} \leftarrow \vec{E}_{\text {highER }}$ and $\vec{G} \leftarrow \vec{G} \backslash \vec{E}_{h i g h E R}$.

3. Partition the edges of $\vec{G}$ into unit weight graphs times a power of 2 , and denote the graph with edge-weights $2^{i}$ as $\vec{G}_{i}$. The output of this step will be $\vec{G}_{1}, \ldots, \vec{G}_{s}$ where $s=O(\log n)$.

4. For each $\vec{G}_{i}$ :

(a) $\left\{C_{i, 1}, \ldots, C_{i, t}\right\} \leftarrow$ CycleDecomposition $\left(G_{i}\right)$. Note that $C_{i j}$ is not a directed cycle, but corresponds to a directed cycle $\vec{C}_{i j}$.

(b) $\vec{H} \leftarrow \vec{H}+\vec{G}_{i} \backslash\left(\cup_{j=1}^{t} \vec{C}_{i j}\right)$.

(c) For each cycle $\vec{C}_{i j}$ :

i. With probability $1 / 2$, add all its clockwise edges with twice their weight to $\vec{H}$; and with probability $1 / 2$, add all its counterclockwise edges with twice their weight to $\vec{H}$.

5. Return $\vec{H}$.

Now, using Lemma 5.2, we can construct a sparsifier of $\vec{G}$ with at most (15/16) $m$ edges: 
Lemma 5.7. Given an Eulerian directed graph $\vec{G}$ with edge weights being integral powers of 2, a 2-approximate estimate $\boldsymbol{r}$ of effective resistances in $G$, and a cycle decomposition routine CycleDecomposition, the algorithm DirectedSparsifyOnce (with pseudocode shown in Algorithm (4) outputs in $O(m)+\mathrm{T}_{\text {CycleDecomp }}(m, n)$ time a directed Eulerian graph $\vec{H}$ with edges weights still being powers of 2 such that with high probability,

$$
\left\|\boldsymbol{L}_{G}^{+/ 2}\left(\boldsymbol{L}_{\vec{G}}-\boldsymbol{L}_{\vec{H}}\right) \boldsymbol{L}_{G}^{+/ 2}\right\|_{2} \leq \epsilon
$$

where $\epsilon=\sqrt{\frac{n L^{3} \log n}{m}}$. Furthermore, if $m=\Omega(\widehat{m} \log n)$, the expected number of edges in $\vec{H}$ is at most $(15 / 16) m$ with high probability.

Proof. First, note that whether a cycle $\vec{C}$ is sampled as clockwise or counterclockwise, the difference between the in-degrees and out-degrees of the vertices does not change. Hence, the graph remains Eulerian. Also, doubling a power of 2 still gives a power of 2, so we get that $\vec{H}$ is still an Eulerian graph with edge weights being powers of 2 .

Next, we prove that the resulting graph $\vec{H}$ is a good approximation of $\vec{G}$. The difference between these two graphs comes from the cycles produced by CyCleDecomposition. Let these cycles be $\left\{\vec{C}_{i}\right\}$. We consider fitting the terms into the requirements of the matrix concentration bound from Lemma 5.2 .

For a cycle $\vec{C}_{i}$, recall that the algorithm samples either $2 \vec{C}_{i}^{\mathrm{CW}}$ or $2\left(\vec{C}_{i} \backslash \vec{C}_{i}^{\mathrm{CW}}\right)$, each with probability $1 / 2$. Let $\boldsymbol{w}_{i}$ be the edge weight of $\vec{C}_{i}$. (Note that we run CyCLEDECOMPosition on equal weighted graphs. $\boldsymbol{w}_{i}$ here must be a power of 2 but we will not use that fact.) Let $\rho / \boldsymbol{w}_{i}$ be an upper bound for the effective resistance of every edge in $\vec{C}_{i}$.

Now, let $\boldsymbol{Y}_{i}$ be the deviation on $i^{\text {th }}$ cycle:

$$
\boldsymbol{Y}_{i}:=\boldsymbol{L}_{G}^{+/ 2}\left(\boldsymbol{L}_{\vec{D}_{i}}-\boldsymbol{L}_{\vec{C}_{i}}\right) \boldsymbol{L}_{G}^{+/ 2}
$$

where

$$
\vec{D}_{i}= \begin{cases}2 \vec{C}_{i}^{\mathrm{CW}} & \text { w.p. } 1 / 2, \\ 2\left(\vec{C}_{i} \backslash \vec{C}_{i}^{\mathrm{CW}}\right) & \text { w.p. } 1 / 2 .\end{cases}
$$

We use $\boldsymbol{X}_{i}^{\text {CW }}$ to denote the deviation on $\vec{C}_{i}$, when it is sampled as clockwise, i.e., $\vec{D}_{i}=2 \vec{C}_{i}$ :

$$
\boldsymbol{X}_{i}^{\mathrm{CW}}:=\boldsymbol{L}_{G}^{+/ 2}\left(2 \boldsymbol{L}_{\vec{C}_{i}^{\mathrm{CW}}}-\boldsymbol{L}_{\vec{C}_{i}}\right) \boldsymbol{L}_{G}^{+/ 2}
$$

Equivalently, we use $\boldsymbol{X}_{i}^{\mathrm{CCW}}$ to denote the deviation on $\vec{C}_{i}$, when it is sampled as counterclockwise. Naturally,

$$
\boldsymbol{Y}_{i}= \begin{cases}\boldsymbol{X}_{i}^{\mathrm{CW}} & \text { w.p. 1/2, } \\ \boldsymbol{X}_{i}^{\mathrm{CCW}} & \text { w.p. 1/2. }\end{cases}
$$

Using our bounds from Lemma 5.4, we have

$$
\left\|\boldsymbol{X}_{i}^{\mathrm{CW}}\right\|_{2} \leq \sqrt{O\left(L^{3} \cdot \rho\right) \cdot \lambda_{\max }\left(\boldsymbol{L}_{G}^{+/ 2} \boldsymbol{L}_{C_{i}} \boldsymbol{L}_{G}^{+/ 2}\right)} .
$$


Since every edge in $C_{i}$ has a leverage score of at most $\rho$, and using the fact that $C$ has at most $L$ edges,

$$
\left\|\boldsymbol{X}_{i}^{\mathrm{CW}}\right\|_{2} \leq O\left(\sqrt{L^{4} \rho^{2}}\right)=O\left(L^{2} \rho\right)
$$

where $\boldsymbol{w}_{i}$ is the edge weight of $\vec{C}_{i}$ We can get the same bound for $\boldsymbol{X}_{i}^{\mathrm{CCW}}$ by a symmetric version of Lemma 5.4 .

To bound the variance term

$$
\left\|\sum_{i} \mathbb{E}\left[\boldsymbol{Y}_{i}^{\top} \boldsymbol{Y}_{i}\right]\right\|_{2}
$$

by Lemma 5.4, both $\left(\boldsymbol{X}_{i}^{\mathrm{CW}}\right)^{\top} \boldsymbol{X}_{i}^{\mathrm{CW}}$ and $\left(\boldsymbol{X}_{i}^{\mathrm{CCW}}\right)^{\top} \boldsymbol{X}_{i}^{\mathrm{CCW}}$ are bounded above by

$$
w_{i} \cdot O\left(L^{3} \cdot \rho\right) \cdot \boldsymbol{L}_{G}^{+/ 2} \boldsymbol{L}_{C_{i}} \boldsymbol{L}_{G}^{+/ 2}
$$

From the definition of $\boldsymbol{Y}_{i}$, and summing over all $i$,

$$
\sum_{i} \mathbb{E}\left[\boldsymbol{Y}_{i}^{\top} \boldsymbol{Y}_{i}\right] \leq O\left(L^{3} \cdot \rho\right) \cdot \sum_{i} \boldsymbol{L}_{G}^{+/ 2} \boldsymbol{L}_{C_{i}} \boldsymbol{L}_{G}^{+/ 2}
$$

Since the cycles are edge disjoint, we have

$$
\sum_{i} \boldsymbol{L}_{C_{i}} \preceq \boldsymbol{L}_{G} .
$$

Composing on either side with $\boldsymbol{L}_{G}^{+/ 2}$ gives

$$
\sum_{i} \boldsymbol{L}_{G}^{+/ 2} \boldsymbol{L}_{C_{i}} \boldsymbol{L}_{G}^{+/ 2} \preceq \boldsymbol{I}
$$

and hence,

$$
\left\|\sum_{i} \mathbb{E}\left[\boldsymbol{Y}_{i}^{\top} \boldsymbol{Y}_{i}\right]\right\|_{2} \leq O\left(L^{3} \cdot \rho\right) .
$$

Similarly,

$$
\left\|\sum_{i} \mathbb{E}\left[\boldsymbol{Y}_{i} \boldsymbol{Y}_{i}^{\top}\right]\right\|_{2} \leq O\left(L^{3} \cdot \rho\right) .
$$

Now, by taking

$$
\rho=\frac{4 n}{m},
$$

Lemma 5.2 gives concentration with high probability.

Next we bound the number of edges in $\vec{H}$ :

1. $\leq m / 2$ edges with low leverage score.

By the same reason as the undirected case (Section 4), since $\boldsymbol{r}_{e}$ is a 2-approximate estimate of effective resistance, by Foster's theorem, the average value of $\boldsymbol{w}_{e} \boldsymbol{r}_{e}$ of all edges is at most $2(n-1) / m$. So at most half of the edges in $\vec{G}$ have $\boldsymbol{w}_{e} \boldsymbol{r}_{e} \geq \frac{4 n}{m}$. 
2. $\leq m / 4$ edges not in cycles.

At most $O(k \log n)$ edges are not in the cycles since $i \leq O(\log n)$. If $m=\Omega(k \log n)$, this number does not exceed $m / 4$.

This leaves at least $m / 4$ edges in cycles.

3. The expected fraction of cycle edges that are added to $\vec{H}$ is $1 / 2$ in the last step. As there are at least $m / 4 L$ cycles and the length of each of the cycles is bounded by $L$, as long as $L=n^{o(1)}$ and $m=\Omega(n)$, by a Chernoff bound, with high probability, at most $3 / 4$ of the cycle edges are added to $\vec{H}$.

We conclude that with high probability, the number of edges in $\vec{H}$ is at most $(15 / 16) \mathrm{m}$.

The running time is dominated by the cycle decomposition step. Because these graphs are edgedisjoint, the super-additivity property of $\operatorname{T}_{\text {CycleDecomp }}(\cdot, n)$ from Equation 2 gives that the total cost of obtaining these decompositions is $\mathrm{T}_{\text {CycleDecomp }}(m, n)$. Also, there are $O(\log n) \leq O(m)$ such graphs, the overhead from handling the different copies is a lower order term.

Finally, by repeating DiRectedSparsifyOnce a number of times, we can reduce the number of edges to almost linear in the number of vertices.

Proof of Theorem 5.1. First we specify the condition under which our guarantees on (1) number of edges, (2) running time and (3) approximation hold.

Because the number of edges reduces by a constant factor with high probability, we know that with high probability, the number of edges reduces by a constant factor in each of the first $O\left(\log \frac{m_{0}}{n}\right)$ rounds. ( $m_{0}$ is the number of edges initially in $\vec{G}$.)

By Lemma 5.7, the resulting graph of DiRECTEDSPARSIFyOnCEapproximates the given graph with high probability. Thus, with high probability, each of the $O\left(\log \frac{m_{0}}{n}\right)$ rounds of DIRECTEDSPARSIFYONCEproduces a valid approximation of the result of its previous round.

Next we consider the case where each of the $O\left(\log \frac{m_{0}}{n}\right)$ rounds of DiRECTEDSPARSIFYOncEreduces the number of edges by a factor of $1 / 16$, and also produces a valid approximation. We have proven that this happens with high probability.

As the number of edges reduces geometrically, the total error, which is bounded by the sum of the errors in all iterations, is bounded up to a constant factor by the error in the last round:

$$
O\left(\sqrt{\frac{n L^{3} \log n}{m}}\right)
$$

where $m$ is the number of edges in the last round. Since the algorithm stops with

$$
m=\Omega\left(n L^{3} \varepsilon^{-2} \log n\right),
$$

picking appropriate constants, this implies that the final error is bounded by $\varepsilon$.

Since our effective resistance estimates depend on $L_{G}$, this small error proves that our initial 1.5-approximate estimate effective resistances remain 2-approximate throughout the algorithm.

The time bound follows from repeating DirectedSPARsifyOnce, and an added $O\left(m \log ^{2} n\right)$ time for computing effective resistance estimates SS11, KLP12]. Note that the number of edges 
increases by a factor of $O(\log n)$ due to the initial splitting into powers of 2 on Line 1 of Algorithm 3 , Then the edge counts are geometrically decreasing, and we bound the overall cost by:

$$
\begin{aligned}
& O\left(m \log ^{2} n\right)+\sum_{i=0}^{O(\log n)} \mathrm{T}_{\text {Cýledecomp }}\left(O\left(\left(\frac{15}{16}\right)^{i} m \log n\right), n\right) \\
& \leq O\left(m \log ^{2} n\right)+\mathrm{T}_{\text {Cycledecomp }}\left(O\left(\sum_{i=0}^{O(\log n)}\left(\frac{15}{16}\right)^{i} m \log n\right), n\right) \\
& \leq O\left(m \log ^{2} n\right)+\mathrm{T}_{\text {Cýlededomp }}(O(m \log n), n) .
\end{aligned}
$$

where the inequality once again follows from the super-additivity assumption of $\mathrm{T}_{\text {CycleDecomp }}(\cdot, n)$ from Equation 2.

\section{Graphical Spectral Sketches and Resistance Sparsifiers}

In this section, we show that every graph has a sparse spectral-sketch with about $n^{1+o(1)} \epsilon^{-1}$ that preserves the quadratic form of the graph Laplacian and its inverse to $1 \pm \epsilon$ with high probability for a fixed vector. The main result in this section is:

Theorem 6.1. Given an undirected weighted graph $G$, a parameter $\varepsilon$, and a cycle decomposition routine CycleDecomposition, the algorithm SpectralSketch (Algorithm 6) returns in

$$
\widetilde{O}(m)+\mathrm{T}_{\text {CyCleDecomp }}(O(m \log n), n)
$$

time a graph $H$ with $\widetilde{O}\left(\widehat{m}+n L \varepsilon^{-1}\right)$ edges, such that with high probability,

1. $H$ is a $\sqrt{\epsilon}$-sparsifier of $G, \boldsymbol{L}_{G} \approx{ }_{\sqrt{\epsilon}} \boldsymbol{L}_{H}$,

2. for any fixed vector $\boldsymbol{x}$, the quadratic form in $\boldsymbol{x}$ is approximately preserved, $\boldsymbol{x}^{\top} \boldsymbol{L}_{H} \boldsymbol{x} \approx_{\varepsilon}$ $\boldsymbol{x}^{\top} \boldsymbol{L}_{G} \boldsymbol{x}$, and

3. for any fixed vector $\boldsymbol{x}$, the inverse quadratic form in $\boldsymbol{x}$ is approximately preserved, $\boldsymbol{x}^{\top} \boldsymbol{L}_{H}^{+} \boldsymbol{x} \approx_{\varepsilon}$ $\boldsymbol{x}^{\top} \boldsymbol{L}_{G}^{+} \boldsymbol{x}$.

Note that part 3 of Theorem 6.1 implies Corollary 3.7. Combining Theorem 6.1 with our two cycle decomposition algorithms leads to our main result on graphical sketches (Theorem 3.6). The guarantees of Theorem 6.1 imply Theorem 3.6 when we use ShortCycleDecomposition as the CycleDecomposition algorithm in Algorithm SpectralSketch.

Proof of Theorem [3.6, assuming Theorem 6.1. Using either NAIVECyCleDeCOMPosition or ShorTCycleDecomposition as the algorithm CycleDecomposition, in Algorithm SpectralSketch, the runtime/sketch size tradeoffs are:

1. Using NaiveCycleDecomposition: SpectralSketch runs in $\widetilde{O}(m n)$ time, and returns an $H$ with $\widetilde{O}\left(n \epsilon^{-1}\right)$ edges.

2. Using ShortCycleDecomposition: SpectralSketch runs in $m^{1+o(1)}$ time, and returns an $H$ with $n^{1+o(1)} \varepsilon^{-1}$ edges. 
Using ShortCycleDecomposition gives us the result of Theorem 3.6.

A natural approach towards this result is a better analysis of the degree-preserving sparsification from Section 4, but showing a better error dependency of about $n / m$ (rather than $\sqrt{n / m}$ ). However, we describe a counter example to this approach.

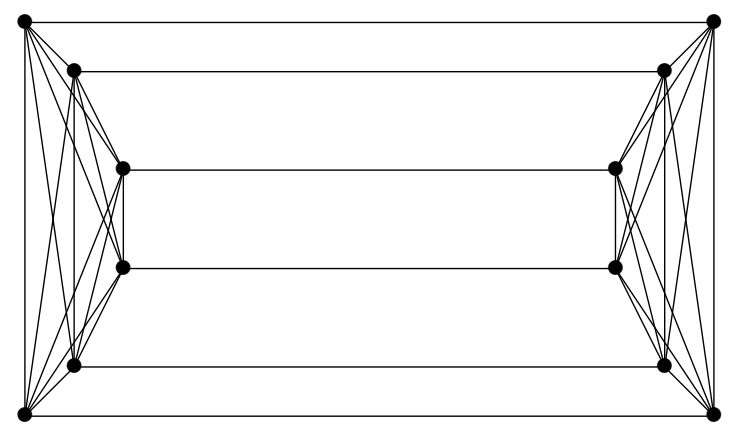

Figure 3: An instance of our counter-example with $n=6$

Consider two cliques of size $n$ connected by a matching of size $n$ (see Figure 3). The effective resistance of each edge, including the edges of the matching are at most $3 / n$. This is because each matching edge has at least $n$ edge-disjoint paths of length 3 connecting its two end points: one path through each edge of the matching using the clique edges. We can also couple these edges into short cycles of length 4, consisting of two matching edges and two clique edges. Now consider a test vector that is 0 on the first clique, and 1 on the second clique. Randomly sampling the matching edges incurs a variance which is about $\sqrt{n}$, so even though all the edges that we sample have resistance at most $3 / n$, the accuracy of our sampling process is limited at $1 / \sqrt{n}$.

Instead, we obtain the better dependency on $\varepsilon$ using the expander based sketching ideas from [JS18]. In Section 6.1 we give a bound for a new degree-preserving scheme whose error depends on the degrees instead of the original quadratic form, and has a $\varepsilon$ dependence. We then incorporate expander decompositions in the same manner as [JS18] to give sketches for arbitrary graphs in Section 6.2. Then in Section 6.3, we show that guarantees 1 and 2 of Theorem 6.1 imply approximation of resistances, proving Corollary 3.7 .

\subsection{Bounding Variance in Terms of Degrees}

We first analyze the errors of an analog of the degree-preserving sparsification routine in the same manner as Jambulapati and Sidford [JS18]. Specifically, we obtain bound on variance, and in turn error, in terms of the degrees of the vertices. This dependency on degrees leads to a slight modification of the algorithm, in that it no longer samples edges incident to low degree vertices. Pseudocode of this routine is in Algorithm 5.

Lemma 6.2. Given a unit weighted simple graph $G$, a parameter $\alpha$, and a cycle decomposition routine CycleDecomposition, the algorithm DecomposeAndSAmple returns a graph $H$ with at most

$$
\widehat{m}+n \alpha+(3 / 4) m
$$


Algorithm 5 DecomposeAndSample $(G, \alpha$, CycleDecomposition)

Input: An undirected simple graph $G$ with all edges having the same weight (WOLOG 1), Degree threshold $\alpha$,

Cycle decomposition routine CyCleDecomposition.

Output: Approximation $H$ with edge weights that are either 1 or 2.

1. Let $V_{b i g}$ be the vertices with degree at least $\alpha$ in $G$.

2. Let $\hat{G}$ be a bipartition of $G\left[V_{b i g}\right]$ with at least half the edges of $G\left[V_{b i g}\right]$.

3. $\left\{C_{1} \ldots C_{t}\right\} \leftarrow \operatorname{CycleDecomposition~}(\hat{G})$.

4. Initialize $H \leftarrow G \backslash\left\{C_{1} \cup C_{2} \ldots \cup C_{t}\right\}$.

5. For each cycle $C_{i}$ :

(a) With probability $1 / 2$, add all the odd indexed edges of $C_{i j}$ into $H$ with weights doubled, otherwise add all the even indexed edges into $H$ with weights doubled.

6. Return $H$.

edges in $O\left(m+\mathrm{T}_{\text {CycleDecomp }}(m, n)\right)$ time, such that for any vector $\boldsymbol{x}$, we have with high probability

$$
\left|\boldsymbol{x}^{\top} \boldsymbol{L}_{H} \boldsymbol{x}-\boldsymbol{x}^{\top} \boldsymbol{L}_{G} \boldsymbol{x}\right| \leq \alpha^{-1} \cdot O(\log n) \cdot \sqrt{L} \cdot \sum_{u} \boldsymbol{d}_{G, u}\left(\boldsymbol{x}_{u}-\widehat{x}\right)^{2}
$$

where $\boldsymbol{d}_{G, u}$ is the degree of vertex $u$ in $G$, and $\widehat{x}$ is an arbitrary scalar.

Our proof utilizes concentration bounds for sums of scalars, specifically Bernstein bounds. We state a slight modification of Bernstein's Inequality:

Lemma 6.3 (Bernstein's Inequality). Let $X_{1} \ldots X_{t}$ be independent random variables such that $\mathbb{E}\left[X_{i}\right]=0$, and for some parameter $\theta$, we have $\left|X_{i}\right| \leq \theta$, and $\sum_{i=1}^{t} \mathbb{E}\left[X_{i}^{2}\right] \leq \theta^{2}$. Then with high probability we have $\left|\sum_{i=1}^{t} X_{i}\right| \leq O(\log n) \cdot \theta$.

The above formulation comes from [CL06], which is obtained by combining Theorems 8.8 and 8.9 in that document with $E\left(X_{i}\right)=0, \lambda=O(\log n) \cdot \theta$, and $\|X\|=M=\theta$, for some parameter $\theta$.

We utilize Bernstein's inequality to prove the main lemma for this section (Lemma 6.2), by creating one $X_{i}$ per cycle.

Proof. (of Lemma 6.2) For each cycle $C_{i}$, let $\widetilde{C}_{i}$ denote the result of sampling either the odd or even indexed edges. We define the random variable $X_{i}$ as

$$
X_{i}:=\boldsymbol{x}^{\top} \boldsymbol{L}_{C_{i}} \boldsymbol{x}-\boldsymbol{x}^{\top} \boldsymbol{L}_{\widetilde{C}_{i}} \boldsymbol{x} .
$$

This is a mean 0 random variable because the expected weight of each edge in $\widetilde{C}$ is 1 . Now, suppose the vertices along $C_{i}$ are $u_{i, 1} \ldots u_{i, n_{i}}$ for some even $n_{i}=\left|C_{i}\right|$. Note that for an edge $u_{i} u_{i+1}$, we can write its corresponding term in the quadratic form, $\left(\boldsymbol{x}_{u_{i, j}}-\boldsymbol{x}_{u_{i, j+1}}\right)^{2}$ as

$$
\left(\boldsymbol{x}_{u_{i, j}}-\widehat{x}\right)^{2}+\left(\boldsymbol{x}_{u_{i, j+1}}-\widehat{x}\right)^{2}-2\left(\boldsymbol{x}_{u_{i, j}}-\widehat{x}\right) \cdot\left(\boldsymbol{x}_{u_{i, j+1}}-\widehat{x}\right)
$$


We use this identity to express $X_{i}$. Since the degree of each vertex in $C_{i}$ is preserved when we sample $\widetilde{C}_{i}$, the first two terms in the above expression cancel out for $\boldsymbol{L}_{C_{i}}$ and $\boldsymbol{L}_{\widetilde{C}_{i}}$, and only the third term remains in $X_{i}$. In the case where we $\widetilde{C}_{i}$ is all the odd indexed edges with double their weights, we get

$$
X_{i}=-2 \sum_{\substack{j=1 \\ j \sim \text { odd }}}^{n_{i}}\left(\boldsymbol{x}_{u_{i, j}}-\widehat{x}\right) \cdot\left(\boldsymbol{x}_{u_{i, j+1}}-\widehat{x}\right)+2 \sum_{\substack{j=2 \\ j \sim \text { even }}}^{n_{i}}\left(\boldsymbol{x}_{u_{i, j}}-\widehat{x}\right) \cdot\left(\boldsymbol{x}_{u_{i, j+1}}-\widehat{x}\right) .
$$

The case where we pick only the even edges is identical, but with signs flipped. Thus, in either case, we have

$$
\left|X_{i}\right| \leq 2 \sum_{j=1}^{n_{i}}\left|\left(\boldsymbol{x}_{u_{i, j}}-\widehat{x}\right) \cdot\left(\boldsymbol{x}_{u_{i, j+1}}-\widehat{x}\right)\right| .
$$

Using these bounds, we invoke Bernstein's inequality from Lemma 6.3 on these variables with

$$
\theta=2 \cdot \alpha^{-1} \cdot \sqrt{L} \cdot \sum_{u \in C_{i}} \boldsymbol{d}_{G, u}\left(\boldsymbol{x}_{u}-\widehat{x}\right)^{2}
$$

for which we need to check the bounds on $\left|X_{i}\right|$ and $\sum_{i} \mathbb{E}\left[X_{i}^{2}\right]$. For the bound on $\left|X_{i}\right|$, the arithmetic-geometric mean (AM-GM) inequality gives

$$
2\left|\left(\boldsymbol{x}_{i, j}-\widehat{x}\right) \cdot\left(\boldsymbol{x}_{u_{i, j+1}}-\widehat{x}\right)\right| \leq\left(\boldsymbol{x}_{i, j}-\widehat{x}\right)^{2}+\left(\boldsymbol{x}_{u_{i, j+1}}-\widehat{x}\right)^{2},
$$

so the overall sum is at most

$$
2 \sum_{u \in C_{i}}\left(\boldsymbol{x}_{u}-\widehat{x}\right)^{2} \leq \theta
$$

since all the degrees are at least $\alpha$.

For the variance term, the Cauchy-Schwarz inequality gives for each $X_{i}$,

$$
\mathbb{E}\left[X_{i}^{2}\right] \leq\left(\sum_{j=1}^{n_{i}} 2\left|\left(\boldsymbol{x}_{u_{i, j}}-\widehat{x}\right) \cdot\left(\boldsymbol{x}_{u_{i, j+1}}-\widehat{x}\right)\right|\right)^{2} \leq 4 n_{i} \cdot \sum_{j=1}^{n_{i}}\left(\boldsymbol{x}_{u_{i, j}}-\widehat{x}\right)^{2}\left(\boldsymbol{x}_{u_{i, j+1}}-\widehat{x}\right)^{2}
$$

Since $G$ is a simple graph, the sum over all these terms can in turn be upper bounded by the sum over all possible pairs of vertices, and factorized:

$$
\sum_{i=1}^{t} \mathbb{E}\left[X_{i}^{2}\right] \leq 4 L \cdot \sum_{u \in V_{b i g}} \sum_{v \in V_{b i g}}\left(\boldsymbol{x}_{u}-\widehat{x}\right)^{2}\left(\boldsymbol{x}_{v}-\widehat{x}\right)^{2}=4 L \cdot\left(\sum_{u \in V_{b i g}}\left(\boldsymbol{x}_{u}-\widehat{x}\right)^{2}\right)^{2} \leq \theta^{2}
$$

where the last inequality follows from all vertices in $V_{b i g}$ having degree at least $\alpha$.

The result then follows from Lemma 6.3.

We remark again that the proof, specifically the factorization of variance, crucially depends on $G$ being a simple graph. 


\subsection{Incorporating Expander Partitioning}

We now turn the error guarantees from Section 6.1, specifically Lemma 6.2 to one that works for general graphs. A key observation in [JS18] is that this sum of vertex-degree terms can be upper bounded by $\boldsymbol{x}^{\top} \boldsymbol{L} \boldsymbol{x}$ via Cheeger's inequality. However, they explicitly renormalize the vector $\boldsymbol{x}$ against the degrees of each piece of the expander. We show instead that these guarantees extend to Laplacians directly with a loss depending on the conductance of the graph.

Definition 6.4. Given an unweighted undirected graph $G$ and a subset of vertices $S$, the conductance of $S$ is

$$
\min _{\widehat{S} \subseteq S} \frac{|E(\widehat{S}, S \backslash \widehat{S})|}{\min \left\{\sum_{u \in \widehat{S}} \boldsymbol{d}_{u}, \sum_{u \in S \backslash \widehat{S}} \boldsymbol{d}_{u}\right\}},
$$

where $\boldsymbol{d}_{u}$ is the degree of $u$ in $G$, and $E(A, B)$ denotes the set of edges with one end point in $A$ and another in $B$.

Note that the conductance of a subgraph $G(S)$ is defined not w.r.t the degrees in $G(S)$, but still the degree of $G$. This is crucial for combining our routine with graph sparsification in Section 7.2 , Cheeger's inequality can also be stated with respect to this subgraph case, giving:

Lemma 6.5 (Cheeger's inequality, AM85]). In any graph $G$, for any subset $S$ with conductance $\phi$, we have

$$
\lambda_{2}\left(\boldsymbol{D}_{S}^{-1 / 2} \boldsymbol{L}_{G[S]} \boldsymbol{D}_{S}^{-1 / 2}\right) \geq \frac{1}{2} \phi^{2},
$$

where $\boldsymbol{L}_{G[S]}$ is the Laplacian matrix of the subgraph of $G$ induced by $S$, and $\boldsymbol{D}_{S}$ is the minor of $\boldsymbol{D}$ restricted to the vertices in $S$.

This formulation is tailored towards expander partitions as they were used in graph sparsification by Spielman and Teng [ST11b]. It can be obtained from the more standard form of Cheeger's inequality by adding self loops at the vertices so their degrees match.

Lemma 6.6. If a unit weighted, undirected graph $G$ has a subset $S$ of vertices with conductance at least $\phi$, then for any vector $\boldsymbol{x}$ we have

$$
\sum_{u v \in E(G[S])}\left(\boldsymbol{x}_{u}-\boldsymbol{x}_{v}\right)^{2} \geq \frac{1}{2} \phi^{2} \sum_{u \in S} \boldsymbol{d}_{u}\left(\boldsymbol{x}_{u}-\widehat{x}\right)^{2},
$$

where $\widehat{x}=\sum_{v}\left(\boldsymbol{d}_{v} /\|\boldsymbol{d}\|_{1}\right) \boldsymbol{x}_{v}$.

Proof. Since Lemma 6.5 takes any degrees, we drop the subscript in $S$ and work with any $\boldsymbol{L}$ and $\boldsymbol{d}$ pair.

Denote $\boldsymbol{d}^{1 / 2}$ as the vector $\left(\sqrt{d_{1}}, \sqrt{d_{2}}, \cdots\right)$ w.r.t. the degree sequence $\boldsymbol{d}$. Then, define $\boldsymbol{\Pi}_{\perp \boldsymbol{d}^{1 / 2}}$ as the orthogonal projection matrix against the unit vector $\boldsymbol{d}^{1 / 2} /\|\boldsymbol{d}\|_{1}^{1 / 2}$, i.e.,

$$
\boldsymbol{\Pi}_{\perp \boldsymbol{d}^{1 / 2}}=\boldsymbol{I}-\frac{\boldsymbol{d}^{1 / 2} \boldsymbol{d}^{1 / 2^{\top}}}{\|\boldsymbol{d}\|_{1}} .
$$


$\boldsymbol{D}^{-1 / 2} \boldsymbol{L} \boldsymbol{D}^{-1 / 2}$ and $\boldsymbol{\Pi}_{\perp \boldsymbol{d}^{1 / 2}}$ share the same null space represented by $\boldsymbol{d}^{1 / 2}$ as $\boldsymbol{D}^{-1 / 2} \boldsymbol{d}^{1 / 2}=\overrightarrow{1}$. Hence, the eigenvalue condition from Cheeger's inequality 6.5 gives

$$
\boldsymbol{D}^{-1 / 2} \boldsymbol{L} \boldsymbol{D}^{-1 / 2} \succeq \frac{1}{2} \phi^{2} \boldsymbol{\Pi}_{\perp \boldsymbol{d}^{1 / 2}}
$$

Thus, for any $\boldsymbol{x}$, composing by $\boldsymbol{D}^{1 / 2} \boldsymbol{x}$ on both sides gives us

$$
\boldsymbol{x}^{\top} \boldsymbol{L} \boldsymbol{x} \geq \frac{1}{2} \phi^{2} \boldsymbol{x}^{\top} \boldsymbol{D}^{1 / 2} \boldsymbol{\Pi}_{\perp \boldsymbol{d}^{1 / 2}} \boldsymbol{D}^{1 / 2} \boldsymbol{x}=\frac{1}{2} \phi^{2} \boldsymbol{x}^{\top}\left(\boldsymbol{D}-\frac{\boldsymbol{d} \boldsymbol{d}^{\top}}{\|\boldsymbol{d}\|_{1}}\right) \boldsymbol{x} .
$$

Or equivalently,

$$
\sum_{u v \in E}\left(\boldsymbol{x}_{u}-\boldsymbol{x}_{v}\right)^{2} \geq \frac{1}{2} \phi^{2}\left(\boldsymbol{x}^{\top} \boldsymbol{D} \boldsymbol{x}-\frac{\boldsymbol{x}^{\top} \boldsymbol{d} \boldsymbol{d}^{\top} \boldsymbol{x}}{\|\boldsymbol{d}\|_{1}}\right)=\frac{1}{2} \phi^{2} \sum_{u} \boldsymbol{d}_{u}\left(\boldsymbol{x}_{u}-\widehat{x}\right)^{2} .
$$

The lack of restrictions on the vector $\boldsymbol{x}$ makes it significantly simpler to sum this guarantee across a number of expanders. Specifically, we invoke the following routine for partitioning graphs into pieces contained in expanders.

Lemma 6.7 ([ST11b] Lemma 32 in $[$ KLOS14] $)$. There is an algorithm ExPANDERDECOMPOSE $(G, \phi)$ that for any unit weighted, undirected graph $G$ with $n$ vertices and $m$ edges and any parameter $\phi>0$, returns with an overhead $\gamma_{\mathrm{ST}}(n)$ that's upper bounded by $\log ^{O(1)} n$ a partition of the vertices of $G$ into

$$
V=\hat{S}_{1} \bullet \hat{S}_{2} \bullet \ldots \cup \hat{S}_{k}
$$

in $\widetilde{O}\left(m \phi^{-2}\right)$ time such that

1. the number of edges on the boundary of all $S_{i}$ 's is at most $\gamma_{\mathrm{ST}}(n) \phi m$,

2. each $\hat{S}_{i}$ is contained in some subset $S_{i} \supseteq \hat{S}_{i}$ such that

(a) the conductance of $S_{i}$ (w.r.t. $G$ ) is at least $\phi^{2}$,

(b) each vertex belongs to at most $O(\log n)$ of the $S_{i}$ s, which in turn implies

$$
\sum_{i} \boldsymbol{L}_{G\left[S_{i}\right]} \preceq O(\log n) \boldsymbol{L}_{G}
$$

Repeatedly running this then gives the overall algorithm, whose pseudocode is in Algorithm 6. Its guarantees come from repeatedly invoking Lemma 6.2 on the pieces given by the expanders.

Proof. (of Theorem 6.1, Parts 1 and 2) We first bound the behavior of each iteration of the inner loop in SpectralSketch.

For the spectral approximation guarantees between $G$ and $H$, Cheeger's inequality (Lemma 6.5) gives

$$
\boldsymbol{L}_{G\left[S_{i}\right]} \succeq \frac{1}{2} \phi^{4} \boldsymbol{\Pi}_{\perp \overrightarrow{1}_{S}} \boldsymbol{D}_{S} \boldsymbol{\Pi}_{\perp \overrightarrow{1}_{S}},
$$


Algorithm 6 SpectralSketch $(G, \varepsilon$, CyCleDeComposition)

Input: undirected graph $G$ with positive integer, poly bounded edge weights

Error $\varepsilon>0$

Cycle decomposition routine CyCleDeComposition.

Output: Approximation $H$.

1. Let $\alpha=32 \varepsilon^{-1} L \gamma_{\mathrm{ST}}(n)^{4} \log ^{4} n$;

2. Decompose $G$ into $G_{1}, G_{2} \ldots$ where all edge weights in $G_{i}$ are $2^{i}$.

3. Repeat $O(\log n)$ times:

(a) Initialize $H$ as empty.

(b) For each $G_{i}$,

i. $\left\{\widehat{S}_{i 1}, \widehat{S}_{i 2}, \ldots\right\} \leftarrow \operatorname{ExpAndERDECOMPOSE}\left(G_{i}, \frac{1}{2 \gamma_{\mathrm{ST}}(n)}\right)$.

ii. Add the edges between pieces to $H$, i.e., $H \leftarrow H \cup G_{i} \backslash\left\{\widehat{S_{i 1}} \cup \widehat{S_{i 2}} \ldots\right\}$.

iii. For each piece $\widehat{S_{i j}}$
A. $\tilde{S}_{i j} \leftarrow$ DecomposeAndSample $\left(\widehat{S_{i j}}, \alpha\right.$, CycleDecomposition $)$
B. Add $\tilde{S}_{i j}$ to $H$

(c) Re-form the $G_{i}$ s from the edges of $H_{i}$.

4. Return $G$.

which means that the effective resistance between two vertices $u$ and $v$ in $S_{i}$ is at most

$$
\frac{2 \phi^{-4}}{\min \left\{\boldsymbol{d}_{u}, \boldsymbol{d}_{v}\right\}} \text {. }
$$

As the only edges sampled in DecomposeAndSampleare the ones with degree at least $\alpha$, the resistances of these edges are bounded by

$$
2 \phi^{-4} \alpha^{-1} \leq 2\left(2 \gamma_{\mathrm{ST}}(n)\right)^{4} \frac{\epsilon}{32 L \gamma_{\mathrm{ST}}(n)^{4} \log ^{4} n} \leq \frac{\epsilon}{L \log ^{4} n}
$$

As the cycle lengths are at most $L$, matrix concentration as stated in Lemma 4.2 gives

$$
\boldsymbol{L}_{G} \approx{ }_{O(\sqrt{\epsilon} / \log n)} \boldsymbol{L}_{H}
$$

with high probability.

For the error in quadratic form, on each of the pieces $\widehat{S}_{i j}$, combining Lemma 6.2

$$
\hat{x}:=\frac{\sum_{u \in S_{i j}} \boldsymbol{d}_{u} \boldsymbol{x}_{u}}{\sum_{v \in S_{i j}} \boldsymbol{d}_{v}},
$$

and Lemma 6.6 gives that, with high probability, we have

$$
\left|\boldsymbol{x}^{\top} \boldsymbol{L}_{\hat{S}_{i j}} \boldsymbol{x}-\boldsymbol{x}^{\top} \boldsymbol{L}_{\tilde{S}_{i j}} \boldsymbol{x}\right| \leq 2 \alpha^{-1} \sqrt{L} \log n \cdot \phi^{-4} \cdot \boldsymbol{x}^{\top} \boldsymbol{L}_{S_{i j}} \boldsymbol{x},
$$


where $\phi^{2}$ is lower bound of the conductance for $S_{i j}$.

Combining these terms, and invoking the guarantees about the overlaps of $S_{i j}$ in Part $2 \mathrm{~b}$ of Lemma 6.7 gives that the total error in sampling $G_{i}$ is at most

$$
32 \alpha^{-1} \sqrt{L} \log ^{2} n \cdot \gamma_{\mathrm{ST}}(n)^{4} \cdot \boldsymbol{x}^{\top} \boldsymbol{L}_{G_{i}} \boldsymbol{x} .
$$

Here, $G_{i}$ refers to the $G_{i}$ variables found in algorithm SpectralSketch. Since we have $O(\log n)$ outer iterations and each of them incurs such a multiplicative error, the total error for the whole algorithm is upper bounded by

$$
32 \alpha^{-1} \sqrt{L} \log ^{3} n \cdot \gamma_{\mathrm{ST}}(n)^{4} \cdot \boldsymbol{x}^{\top} \boldsymbol{L}_{G_{i}} \boldsymbol{x} .
$$

The choice of $\alpha$ then gives an error of at most $(\epsilon / \log n) \boldsymbol{x}^{\top} \boldsymbol{L}_{G_{i}} \boldsymbol{x}$. This bound is not tight, however, our choice of $\alpha$ is constrained by the need for $H$ to be an $\frac{\sqrt{\varepsilon}}{\log n}$ sparsifier for $G$ (Equation (7)). Also, note that the guarantees of DecomposeAndSAmple means that we only add edges whose weights are powers of 2 back. So after each step $H$ can be decomposed back to the $G_{i}$ s without any increases in edge counts.

Now, let $m^{\prime} \stackrel{\text { def }}{=} \sum_{i}\left|E\left(G_{i}\right)\right|$. The choice of $\phi=\frac{1}{2 \gamma_{\mathrm{ST}}(n)}$ for ExpANDERDECOMPOSE means that at least $m^{\prime} / 2$ edges are contained in the $\widehat{S}_{i j}$ s. As the $\widehat{S}_{i j}$ s are vertex-disjoint, and at least half of the edges went into them, we have that $m^{\prime}$ edges get reduced to at most

$$
\tilde{O}\left(\widehat{m}+n \varepsilon^{-1} L\right)+\frac{7}{8} m^{\prime}
$$

edges after one iteration. Treating the first term as a function of $n, m(n)$, we get that as long as $m^{\prime}>10 m(n)$, the edge count decreases by a constant factor after each step. So the $O(\log n)$ outer iterations suffices for bringing the edge count to $O(m(n))$, and we obtain the approximation guarantees by taking this increase in errors into account.

Now, we'd like to bound the runtime of SpectralSketch. To do this, we bound the runtime of a single iteration of the outer loop in that algorithm. Each iteration consists of a call to ExPANDERDecompose on each $G_{i}$, and a call to CyCleDecomposition on each $\widehat{S_{i j}}$. Therefore, the runtime of each iteration of the loop is upper bounded by:

$$
\sum_{i} \tilde{O}\left(\left|E\left(G_{i}\right)\right|\left(2 \gamma_{\mathrm{ST}}(n)\right)^{2}\right)+\sum_{i j} \mathrm{~T}_{\text {CycleDecomp }}\left(\left|E\left(\widehat{S}_{i j}\right)\right|,\left|V\left(\widehat{S}_{i j}\right)\right|\right) .
$$

Note that

$$
\sum_{i j}\left|E\left(\widehat{S}_{i j}\right)\right| \leq \sum_{i}\left|E\left(G_{i}\right)\right|=m^{\prime}
$$

and

$$
\left|V\left(\widehat{S}_{i j}\right)\right| \leq n
$$

Recall that $\gamma_{\mathrm{ST}}(n)$ is upper bounded by $\log ^{O(1)}(n)$, by Lemma 6.7. Since $\mathrm{T}_{\text {CrcleDecomp }}(\cdot, n)$ is super-additive by the assumption in Equation 2, and $\mathrm{T}_{\text {CycleDecomp }}$ is monotonic in $n$, we can bound the runtime of each iteration in the loop by:

$$
\tilde{O}\left(\sum_{i}\left|E\left(G_{i}\right)\right|\right)+\mathrm{T}_{\text {CycleDecomp }}\left(\sum_{i j}\left|E\left(\widehat{S}_{i j}\right)\right|, n\right)
$$




$$
\leq \tilde{O}\left(m^{\prime}\right)+\mathrm{T}_{\text {CyCleDecomp }}\left(m^{\prime}, n\right)
$$

Now it remains to sum this quantity over all iterations of the outer loop in SpeCtRALSketch. Since $m^{\prime}$ decreases geometrically, and the initial value of $m^{\prime}$ is less than $O(m \log n)$, we can once again use the superadditivity property of $T(\cdot, n)$ to bound the runtime of the entire SPECTRALSKETCH algorithm by:

$$
\widetilde{O}(m)+\mathrm{T}_{\text {CycleDecomp }}(O(m \log n), n),
$$

as desired.

The dependence of $\alpha$ on $L$ can be improved by a factor of $\sqrt{L}$ by using the operator version of matrix Chernoff that we will state in Lemma 7.2, However, we omit this improvement in the current version.

\subsection{Converting Guarantees to on Inverses}

We now turn our attention to the quadratic inverse form. Here our proof is by a black-box combination of the $\epsilon$-error guarantees on the quadratic form with a $\sqrt{\epsilon}$ guarantee on matrix approximations.

Lemma 6.8. Suppose $\boldsymbol{P}$ and $\boldsymbol{Q}$ are matrices, and $\boldsymbol{x}$ is a vector such that for some $\varepsilon \in(0,0.1]$ we have:

1. $\boldsymbol{P}$ and $\boldsymbol{Q} \sqrt{\varepsilon}$-approximate each other spectrally $\boldsymbol{P} \approx_{\sqrt{\varepsilon}} \boldsymbol{Q}$, and

2. The quadratic forms of $\boldsymbol{P}^{+} \boldsymbol{x}$ under $\boldsymbol{P}$ and $\boldsymbol{Q}$-approximate each other: (note that $\boldsymbol{x}^{\top} \boldsymbol{P}^{+} \boldsymbol{P} \boldsymbol{P}^{+} \boldsymbol{x}$ simplifies to $\boldsymbol{x}^{\top} \boldsymbol{P}^{+} \boldsymbol{x}$.)

$$
\boldsymbol{x}^{\top} \boldsymbol{P}^{+} \boldsymbol{x} \approx_{\varepsilon}\left(\boldsymbol{P}^{+} \boldsymbol{x}\right)^{\top} \boldsymbol{Q} \boldsymbol{P}^{+} \boldsymbol{x}
$$

Then, we have, $\boldsymbol{x}^{\top} \boldsymbol{Q}^{+} \boldsymbol{x} \approx_{7 \epsilon} \boldsymbol{x}^{\top} \boldsymbol{P}^{+} \boldsymbol{x}$.

Proof. We will show that $\boldsymbol{x}^{\top} \boldsymbol{Q}^{+} \boldsymbol{x}$ is close to

$$
\boldsymbol{x}^{\top}\left(2 \boldsymbol{P}^{+}-\boldsymbol{P}^{+} \boldsymbol{Q} \boldsymbol{P}^{+}\right) \boldsymbol{x}
$$

which will in turn enable us to incorporate the condition on $\boldsymbol{x}^{\top} \boldsymbol{P}^{+} \boldsymbol{Q} \boldsymbol{P}^{+} \boldsymbol{x}$ being close to $\boldsymbol{x}^{\top} \boldsymbol{P}^{+} \boldsymbol{x}$. This holds even in the matrix setting. The condition of

$$
\boldsymbol{P} \approx_{\sqrt{\varepsilon}} \boldsymbol{Q}
$$

by the preservation of approximations under pseudoinverses stated in Fact 2.2 implies

$$
\boldsymbol{Q}^{+} \approx_{\sqrt{\varepsilon}} \boldsymbol{P}^{+}
$$

Composing by $\boldsymbol{Q}^{1 / 2}$ on both sides gives that all the eigenvalues of

$$
\boldsymbol{\Pi}-\boldsymbol{Q}^{1 / 2} \boldsymbol{P}^{+} \boldsymbol{Q}^{1 / 2}
$$

are in the range $\left[e^{-\sqrt{\varepsilon}}-1, e^{\sqrt{\varepsilon}}-1\right]$, where $\boldsymbol{\Pi}$ is the projection matrix onto the column space of $\boldsymbol{P}$ and $\boldsymbol{Q}$. 
Squaring this then gives that all the eigenvalues of

$$
\left(\boldsymbol{\Pi}-\boldsymbol{Q}^{1 / 2} \boldsymbol{P}^{+} \boldsymbol{Q}^{1 / 2}\right)^{2}
$$

are non-negative and no more than $\max \left(\left(e^{-\sqrt{\varepsilon}}-1\right)^{2},\left(e^{\sqrt{\varepsilon}}-1\right)^{2}\right) \leq\left(e^{\sqrt{\varepsilon}}-1\right)^{2} \leq 4 \varepsilon$, where the last inequality follows from the fact that $e^{x} \leq 1+2 x$ for $x \leq 1$. This, when expanded becomes

$$
0 \preceq \boldsymbol{\Pi}-2 \boldsymbol{Q}^{1 / 2} \boldsymbol{P}^{+} \boldsymbol{Q}^{1 / 2}+\boldsymbol{Q}^{1 / 2} \boldsymbol{P}^{+} \boldsymbol{Q} \boldsymbol{P}^{+} \boldsymbol{Q}^{1 / 2} \preceq 4 \varepsilon \boldsymbol{\Pi} .
$$

Moving the $\boldsymbol{\Pi}$ terms outside, composing both sides by $\boldsymbol{Q}^{+1 / 2}$, and flipping signs gives,

$$
\boldsymbol{Q}^{+} \succeq 2 \boldsymbol{P}^{+}-\boldsymbol{P}^{+} \boldsymbol{Q} \boldsymbol{P}^{+} \succeq(1-4 \varepsilon) \boldsymbol{Q}^{+} \succeq e^{-5 \varepsilon} \boldsymbol{Q}^{+}
$$

Here the last inequality utilizes the assumption of $\epsilon \leq 0.1$. Substituting in the vector $\boldsymbol{x}$ then gives:

$$
e^{-5 \varepsilon} \boldsymbol{x}^{\top} \boldsymbol{Q}^{+} \boldsymbol{x} \leq \boldsymbol{x}^{\top}\left(2 \boldsymbol{P}^{+}-\boldsymbol{P}^{+} \boldsymbol{Q} \boldsymbol{P}^{+}\right) \boldsymbol{x} \leq \boldsymbol{x}^{\top} \boldsymbol{Q}^{+} \boldsymbol{x} .
$$

On the other hand, Assumption 2 on the quadratic forms involving $\boldsymbol{x}$, specifically $\boldsymbol{x}^{\top} \boldsymbol{P}^{+} \boldsymbol{x} \approx_{\epsilon}$ $\boldsymbol{x}^{\top} \boldsymbol{P}^{+} \boldsymbol{Q} \boldsymbol{P}^{+} \boldsymbol{x}$ gives

$$
-e^{\epsilon} \boldsymbol{x}^{\top} \boldsymbol{P}^{+} \boldsymbol{x} \leq-\boldsymbol{x}^{\top} \boldsymbol{P}^{+} \boldsymbol{Q} \boldsymbol{P}^{+} \boldsymbol{x} \leq-e^{-\epsilon} \boldsymbol{x}^{\top} \boldsymbol{P}^{+} \boldsymbol{x}
$$

to which we add $2 \boldsymbol{x}^{\top} \boldsymbol{P}^{+} \boldsymbol{x}$ to both sides to obtain

$$
\left(2-e^{\epsilon}\right) \boldsymbol{x}^{\top} \boldsymbol{P}^{+} \boldsymbol{x} \leq 2 \boldsymbol{x}^{\top} \boldsymbol{P}^{+} \boldsymbol{x}-\boldsymbol{x}^{\top} \boldsymbol{P}^{+} \boldsymbol{Q} \boldsymbol{P}^{+} \boldsymbol{x} \leq\left(2-e^{-\epsilon}\right) \boldsymbol{x}^{\top} \boldsymbol{P}^{+} \boldsymbol{x} .
$$

Simplifying this again using the assumption of $\epsilon \leq 0.1$ then gives

$$
e^{-2 \varepsilon} \boldsymbol{x}^{\top} \boldsymbol{P}^{+} \boldsymbol{x} \leq 2 \boldsymbol{x}^{\top} \boldsymbol{P}^{+} \boldsymbol{x}-\boldsymbol{x}^{\top} \boldsymbol{P}^{+} \boldsymbol{Q} \boldsymbol{P}^{+} \boldsymbol{x} \leq e^{2 \varepsilon} \boldsymbol{x}^{\top} \boldsymbol{P}^{+} \boldsymbol{x} .
$$

Combining this with Equation (8), gives $\boldsymbol{x}^{\top} \boldsymbol{P}^{+} \boldsymbol{x} \approx_{7 \epsilon} \boldsymbol{x}^{\top} \boldsymbol{Q}^{+} \boldsymbol{x}$.

The inverse quadratic form bound from Theorem 6.1 Part 3 then follows from the spectral approximation guarantees and quadratic form guarantees from Parts 1 and 2, with a suitable constant factor change in $\epsilon$.

A direct corollary to this theorem is that our graph sketches preserve effective resistances. Taking a union bound over all $n^{2}$ vectors $\chi_{u v}$, we obtain Corollary 3.7 .

\section{Computing Effective Resistances with Better $\varepsilon$-Dependency}

In this section, we give an algorithm for computing the effective resistances of all edges in $m^{1+o(1)} \varepsilon^{-1.5}$ time, proving Theorem 3.8 .

In Section 7.3, we will show that computing effective resistances reduces to sparsifying weighted cliques and bicliques, and further that sparsifying these implicitly reduces (with a polylog overhead) to sparsifying a union of unit weighted bipartite cliques whose sizes are powers of $2, \mathcal{K}_{B, 2^{i}}$. We also show in Section 7.2 that such collections of bicliques interact well with expander decompositions, up to polylog factors.

On the other hand, it's now far harder to require such collections of bicliques to be simple: checking whether a collection of $O(\log n)$ cliques on $\Theta(n)$ vertices is simple is in fact equivalent 
to the orthogonal vectors problem $\left[\mathrm{FMP}^{+} 17\right]$. This is problematic for the variance analysis in, Section [6.1. The analysis, like a similar one from Jambulapati and Sidford [JS18], relies on the graph being simple.

However, note that if we are handling $\mathcal{K}_{B, r}$, a collection of balanced bicliques with size $r$, we can use the value of $r$ to bound the multiplicities of edges in $G\left(\mathcal{K}_{B, r}\right)$. A vertex with degree $d$ in this graph is involved in $d / r$ bicliques, and as a result, each edge has multiplicity at most $d / r$. This drop is sufficient for a reduction of running time by $\varepsilon^{-0.5}$; we handle smaller bicliques by constructing them explicitly instead.

To obtain this running time, it is critical to access implicit representations of dense graphs in time proportional to the number of vertices involved. In particular, bipartite cliques are the most convenient intermediate states for our algorithms because it is easy to sample them in a degree-preserving manner: a matching suffices.

Definition 7.1. We will use $\mathcal{K}$ to denote a collection of cliques and bicliques. In particular:

- $\mathcal{K}_{B}$ denotes a collection of unit weighted bipartite cliques.

- $\mathcal{K}_{B,=}$ denotes a collection of unit weighted balanced bicliques, that is, each biclique has the same number of vertices on each side.

- $\mathcal{K}_{B, r}$ denotes a collection of unit weighted balanced bicliques with $r$ vertices on each side.

We use $G(\mathcal{K})$ to denote the explicit graph formed by the union of the cliques in $\mathcal{K}$. We use $n(\mathcal{K})$ to denote the total number of vertices of all the cliques in the collection, and $m(\mathcal{K})$ to denote the total edge count, taking multiplicities into account. Note that these values are not the same as the total vertices $n$, and the total edges $m$, since the bicliques are not disjoint. Given such a collection of cliques, we can compute the degrees of all the vertices, and in turn $m(\mathcal{K})$ by summing together the sizes of the cliques that a vertex $u$ is involved in.

A major issue that we need to address is that the sum of leverage scores of a sampled matching (as required by Lemma 4.2) can now be very large. Specifically, suppose the graph is one copy of $K_{B, n}$, and we sample it to $s$ matchings, each rescaled to $n / s$. Then the leverage score of a single edge by symmetry is about $\Theta(1 / n)$, which means the total leverage score of a matching is $\Theta(1)$. With the rescaling factor of $n / s$, each sample then has a seemingly prohibitive total leverage score of about $n / s$, necessitating all $n$ samples.

Instead, we utilize a multi-edge version of graph sampling based on the maximum magnitude of a sampled graph against another matrix.

Lemma 7.2. Let $G_{1} \ldots G_{k}$ be distributions over random graphs with expectation $G=\sum_{i} \mathbb{E}\left[G_{i}\right]$ so that for all $i$ we have

$$
\boldsymbol{L}_{G_{i}} \preceq O\left(\frac{\varepsilon^{2}}{\log n}\right) \boldsymbol{L}_{G},
$$

where $\varepsilon \leq 1$. Then with high probability we have $\sum_{i} G_{i} \approx_{\varepsilon} G$.

The preceding lemma is a corollary of the Matrix Chernoff bound from [Tro12].

\subsection{Sketching Unweighted bicliques}

We start with the simplest case: bicliques that all have the same size, and are balanced, that is, have $r$ vertices on each side. The pseudocode of our sampling scheme is in Algorithm 7 , We will bound the convergence of this routine by bounding the variance of each matching sampled. 
Lemma 7.3. Let $K$ be a bipartite clique between $V_{A}$ and $V_{B}$ each of size $r$. Let $H$ be a random matching on these vertices with weight set to $r$. Then for any vector $\boldsymbol{x}$ and any value $\widehat{x}$, we have

$$
\underset{H}{\operatorname{Var}}\left[\boldsymbol{x}^{\top} \boldsymbol{L}_{H} \boldsymbol{x}\right] \leq \sum_{a \in V_{A}, b \in V_{B}} 4 r \cdot\left(\boldsymbol{x}_{a}-\widehat{x}\right)^{2} \cdot\left(\boldsymbol{x}_{b}-\widehat{x}\right)^{2} .
$$

Note that this would imply the variance incurred by SAMPLEMATCHINGS is $\frac{4 r}{s} \sum_{a \in V_{a}, b \in V_{b}}\left(\boldsymbol{x}_{a}-\right.$ $\widehat{x})^{2}\left(\boldsymbol{x}_{b}-\widehat{x}\right)^{2}$, as it averages over $s$ matchings.

Algorithm 7 SampleMatchings $\left(\mathcal{K}_{B, r}, s\right)$

Input: A collection of unit weighted bicliques, each of size $r$. Sampling overhead $s$.

Output: Approximation $H$.

1. Initialize $H \leftarrow \emptyset$.

2. For each $K \in \mathcal{K}_{B, r}$

(a) Repeat $s$ times

i. Add a random matching on the vertices of $K$ to $H$, with weight $\frac{r}{s}$.

3. Return $H$

Proof. For simplicity, we consider the $\widehat{x}=0$ case first. We will use the variables $a, a_{1}$, and $a_{2}$ to index over vertices in $V_{A}$, and $b, b_{1}, b_{2}$ to index over vertices in $V_{B}$.

By construction, $\mathbb{E}\left[\boldsymbol{L}_{H}\right]=\boldsymbol{L}_{K}$. Note that by the degree preserving property,

$$
\underset{H}{\mathbb{E}}\left[\boldsymbol{x}^{\top} \boldsymbol{L}_{H} \boldsymbol{x}\right]-\boldsymbol{x}^{\top} \boldsymbol{L}_{K} \boldsymbol{x}=\underset{H}{\mathbb{E}}\left[\boldsymbol{x}^{\top} \boldsymbol{A}_{H} \boldsymbol{x}\right]-\boldsymbol{x}^{\top} \boldsymbol{A}_{K} \boldsymbol{x},
$$

where $\boldsymbol{A}_{H}$ represents the adjacency matrix of $H$. Therefore,

$$
\underset{H}{\operatorname{Var}}\left[\boldsymbol{x}^{\top} \boldsymbol{L}_{H} \boldsymbol{x}\right]=\underset{H}{\operatorname{Var}}\left[\boldsymbol{x}^{\top} \boldsymbol{A}_{H} \boldsymbol{x}\right] .
$$

Let $X$ denote the random variable $\boldsymbol{x}^{\top} \boldsymbol{A}_{H} \boldsymbol{x}$. We will bound $\operatorname{Var}_{X}[X]=\mathbb{E}_{X}\left[X^{2}\right]-\mathbb{E}_{X}[X]^{2}$ by expanding $\mathbb{E}_{X}\left[X^{2}\right]$ and $\mathbb{E}_{X}[X]^{2}$. Let $\mathcal{M}$ be the set of all matchings mapping $A$ to $B$, and $M$ be one such matching. That is, $M(a)$ over all $a \in A$ gives a permutation in $B$. We have

$$
\begin{aligned}
\underset{H}{\mathbb{E}}\left[X^{2}\right] & =r^{2}\left(\frac{1}{r !} \sum_{M \in \mathcal{M}}\left(\sum_{a} \boldsymbol{x}_{a} \boldsymbol{x}_{M(a)}\right)^{2}\right) \\
& =\frac{r^{2}}{r !} \sum_{M \in \mathcal{M}} \sum_{a} \boldsymbol{x}_{a}^{2} \boldsymbol{x}_{M(a)}^{2}+\frac{r^{2}}{r !} \sum_{M \in \mathcal{M}} \sum_{a_{1} \neq a_{2}} \boldsymbol{x}_{a_{1}} \boldsymbol{x}_{M\left(a_{1}\right)} \boldsymbol{x}_{a_{2}} \boldsymbol{x}_{M\left(a_{2}\right)} .
\end{aligned}
$$

For any $a$ and $b$, there are $(r-1)$ ! matchings that have $M(a)=b$, so the first term simplifies to

$$
\frac{r^{2}}{r !} \sum_{M \in \mathcal{M}} \sum_{a} \boldsymbol{x}_{a}^{2} \boldsymbol{x}_{M(a)}^{2}=r \cdot \sum_{a, b} \boldsymbol{x}_{a}^{2} \boldsymbol{x}_{b}^{2}
$$


Also, for any $a_{1} \neq a_{2}$, and $b_{1} \neq b_{2}$, there are $(r-2)$ ! matchings that have $M\left(a_{1}\right)=b_{1}$ and $M\left(a_{2}\right)=b_{2}$. Therefore the second term simplifies to

$$
\frac{r^{2}}{r !} \sum_{M \in \mathcal{M}} \sum_{a_{1} \neq a_{2}} \boldsymbol{x}_{a_{1}} \boldsymbol{x}_{M\left(a_{1}\right)} \boldsymbol{x}_{a_{2}} \boldsymbol{x}_{M\left(a_{2}\right)}=\frac{r}{r-1} \sum_{a_{1} \neq a_{2}, b_{1} \neq b_{2}} \boldsymbol{x}_{a_{1}} \boldsymbol{x}_{a_{2}} \boldsymbol{x}_{b_{1}} \boldsymbol{x}_{b_{2}} .
$$

On the other hand, the expectation of $X$ evaluates to

$$
\underset{H}{\mathbb{E}}[X]=\sum_{a, b} \boldsymbol{x}_{a} \boldsymbol{x}_{b}=\left(\sum_{a} \boldsymbol{x}_{a}\right)\left(\sum_{b} \boldsymbol{x}_{b}\right) .
$$

Squaring this, and grouping the terms by duplicity in a gives:

$$
\underset{H}{\mathbb{E}}[X]^{2}=\sum_{a, b} \boldsymbol{x}_{a}^{2} \boldsymbol{x}_{b}^{2}+\sum_{a, b_{1} \neq b_{2}} \boldsymbol{x}_{a}^{2} \boldsymbol{x}_{b_{1}} \boldsymbol{x}_{b_{2}}+\sum_{a_{1} \neq a_{2}, b} \boldsymbol{x}_{a_{1}} \boldsymbol{x}_{a_{2}} \boldsymbol{x}_{b}^{2}+\sum_{a_{1} \neq a_{2}, b_{1} \neq b_{2}} \boldsymbol{x}_{a_{1}} \boldsymbol{x}_{a_{2}} \boldsymbol{x}_{b_{1}} \boldsymbol{x}_{b_{2}},
$$

and in turn:

$$
\begin{aligned}
\underset{H}{\operatorname{Var}}[X] & =\underset{X}{\mathbb{E}}\left[X^{2}\right]-\underset{X}{\mathbb{E}}[X]^{2} \\
& =(r-1) \cdot \sum_{a b} \boldsymbol{x}_{a}^{2} \boldsymbol{x}_{b}^{2}-\sum_{a, b_{1} \neq b_{2}} \boldsymbol{x}_{a}^{2} \boldsymbol{x}_{b_{1}} \boldsymbol{x}_{b_{2}}-\sum_{a_{1} \neq a_{2}, b} \boldsymbol{x}_{a_{1}} \boldsymbol{x}_{a_{2}} \boldsymbol{x}_{b}^{2}+\frac{1}{r-1} \sum_{a_{1} \neq a_{2}, b_{1} \neq b_{2}} \boldsymbol{x}_{a_{1}} \boldsymbol{x}_{a_{2}} \boldsymbol{x}_{b_{1}} \boldsymbol{x}_{b_{2}} .
\end{aligned}
$$

We bound each of these terms separately. For the second term, we have $\boldsymbol{x}_{b_{1}}^{2}+\boldsymbol{x}_{b_{2}}^{2} \geq 2\left|\boldsymbol{x}_{b_{1}} \boldsymbol{x}_{b_{2}}\right|$, so we have

$$
-\sum_{a, b_{1} \neq b_{2}} \boldsymbol{x}_{a}^{2} \boldsymbol{x}_{b_{1}} \boldsymbol{x}_{b_{2}} \leq \frac{1}{2} \sum_{a, b_{1} \neq b_{2}} \boldsymbol{x}_{a}^{2}\left(\boldsymbol{x}_{b_{1}}^{2}+\boldsymbol{x}_{b_{2}}^{2}\right) \leq(r-1) \sum_{a, b} \boldsymbol{x}_{a}^{2} \boldsymbol{x}_{b}^{2},
$$

and the third term follows similarly by applying the two-term arithmetic-geometric mean inequality to $\boldsymbol{x}_{a_{1}} \boldsymbol{x}_{a_{2}}$. We can also bound the last term by applying this inequality simultaneously on both $a_{1} a_{2}$ and $b_{1} b_{2}$ :

$$
\begin{aligned}
\frac{1}{r-1} \sum_{a_{1} \neq a_{2}, b_{1} \neq b_{2}} \boldsymbol{x}_{a_{1}} \boldsymbol{x}_{a_{2}} \boldsymbol{x}_{b_{1}} \boldsymbol{x}_{b_{2}} & \leq \frac{1}{4(r-1)} \sum_{a_{1} \neq a_{2}, b_{1} \neq b_{2}}\left(\boldsymbol{x}_{a_{1}}^{2}+\boldsymbol{x}_{a_{2}}^{2}\right)\left(\boldsymbol{x}_{b_{1}}^{2}+\boldsymbol{x}_{b_{2}}^{2}\right) \\
& \leq(r-1)\left(\sum_{a} \boldsymbol{x}_{a}^{2}\right)\left(\sum_{b} \boldsymbol{x}_{b}^{2}\right) .
\end{aligned}
$$

Summing across these four terms gives the lemma statement for $\widehat{x}=0$. The general lemma statement follows from observing that

$$
\boldsymbol{x}^{\top} \boldsymbol{L}_{H} \boldsymbol{x}=(\boldsymbol{x}-\widehat{x} \overrightarrow{1})^{\top} \boldsymbol{L}_{H}(\boldsymbol{x}-\widehat{x} \overrightarrow{1}),
$$

where $\overrightarrow{1}$ is the all ones vector. So $\boldsymbol{x}$ can be replaced by $\boldsymbol{x}-\widehat{x}$ throughout this calculation.

We now bound the overall variance when we sample matchings for a collection of such bicliques. Here, we also need a sampling overhead $s$. We give the following relatively technical lemma involving 
a degree threshold on the vertices that's a more limited analog of Lemma 6.2 in that it gives a w.h.p. bound on the errors of sampling the quadratic form in terms of the degrees involved.

Because we now need to bound $\boldsymbol{L}_{K}$ against $\boldsymbol{L}_{G}$, it is useful to work with the case where all vertices in $K$ have fairly large degree. To narrow down to this setting, we will partition the degrees so that the degrees involved in each biclique boil down to a single parameter. We first show our sampling routine under this restriction, and then discuss how to narrow down to this case by bucketing the vertices in the more general case.

Lemma 7.4. Let $\mathcal{K}_{B, r}$ be a collection of $k$ unit weighted balanced bicliques of size $r$ each, and $V_{S}$ a set of vertices in $G\left(\mathcal{K}_{B, r}\right)$ such that for some $d$ and $\varepsilon$ with $d \geq \varepsilon^{-3 / 2}$ we have:

1. Each vertex in $V_{S}$ has degree at most $d$ in $G\left(\mathcal{K}_{B, r}\right)$.

2. Each biclique $K \in \mathcal{K}_{B, r}$ with $V_{K}=\{A, B\}$ has $A \subseteq V_{S}$ (the mirror case of $B \subseteq V_{S}$ is equivalent to this by swapping the two sides).

The distribution over graphs

$$
H=\operatorname{SampleMatchings}\left(\mathcal{K}_{B, r}, \max \left\{\varepsilon^{-1 / 2}, 4 r \varepsilon^{-1} / d\right\}\right)
$$

gets sampled in $\widetilde{O}\left(n \varepsilon^{-1}+n(\mathcal{K}) \varepsilon^{-1 / 2}\right)$ time, and satisfies the following:

1. $H$ has $\widetilde{O}\left(n \varepsilon^{-1}+n(\mathcal{K}) \varepsilon^{-1 / 2}\right)$ edges.

2. $\mathbb{E}[H]=G\left(\mathcal{K}_{B, r}\right)$.

3. Any graph $M$ (which is a rescaled matching) in the support of $H$ satisfies

$$
\boldsymbol{L}_{M} \preceq \varepsilon d \boldsymbol{I}_{\perp \overrightarrow{1}},
$$

where $\boldsymbol{I}_{S \perp \overrightarrow{1}}$ is the identity matrix on $S$ projected against the all 1 s vector.

4. For any fixed vector $\boldsymbol{x}$ and any $\widehat{x}$, we have with high probability:

$$
\left|\boldsymbol{x}^{\top} \boldsymbol{L}_{H} \boldsymbol{x}-\boldsymbol{x}^{\top} \boldsymbol{L}_{G\left(\mathcal{K}_{B, r}\right)} \boldsymbol{x}\right| \leq O(\log n) \cdot \varepsilon \cdot \sum_{u} d\left(\boldsymbol{x}_{u}-\widehat{x}\right)^{2} .
$$

The condition involving $V_{S}$ is useful because we can only reduce to collections of bicliques incident to small degree vertices, and have less control on the degrees of the other side. Also, the lower bound on edge sampling probabilities is necessary for a proof of spectral approximations using Lemma 4.2. Such operator (instead of vector) approximations are necessary for converting the guarantees from quadratic forms to quadratic inverse forms (and hence resistance approximations) using Lemma 6.8.

Proof. We use $s$ to denote the sample count per clique

$$
s:=\max \left\{\varepsilon^{-1 / 2}, 4 r \varepsilon^{-1} / d\right\} .
$$

For the number of edges sampled, we can sum the two bounds, one per choice of term. 
- Picking $\varepsilon^{-1 / 2}$ matchings per biclique gives an edge count that's $\varepsilon^{-1 / 2}$ times the size of each biclique, summing to a total of $k r \varepsilon^{-1 / 2}=(1 / 2) n(\mathcal{K}) \varepsilon^{-1 / 2}$.

- The $4 r \varepsilon^{-1} / d$ matchings contributes to a total of

$$
\frac{4 r^{2} \varepsilon^{-1}}{d}
$$

sampled edges per biclique. On the other hand, the total number of edges $m(\mathcal{K})$ in $G(\mathcal{K})$ is at most $n d$. Since each biclique has $r^{2}$ edges, the total number of bicliques is at most

$$
\frac{n d}{r^{2}}
$$

which multiplied by the number of matching edges sampled per biclique gives a total of

$$
\frac{n d}{r^{2}} \cdot \frac{4 r^{2} \varepsilon^{-1}}{d} \leq O\left(n \varepsilon^{-1}\right)
$$

To bound the expectation, consider a single biclique $K$. Let $M_{1}, \ldots, M_{s}$ be the sampled matchings. By symmetry, we have $\mathbb{E}\left[M_{i}\right]=K$, so by linearity of expectation we get $\mathbb{E}[H]=G(\mathcal{K})$.

We also need to bound the maximum magnitude of a sample. The rescaling factor of $r / s$ means that the value of each sample picked in some clique $K$ (with a matching $M$ ) is at most

$$
\frac{r}{s} \boldsymbol{x}^{\top} \boldsymbol{L}_{M} \boldsymbol{x}=\frac{r}{s} \sum_{a \in V_{K, A}}\left(\boldsymbol{x}_{a}-\boldsymbol{x}_{M(a)}\right)^{2} \leq \frac{2 r}{s} \sum_{a \in V_{K, A}}\left(\boldsymbol{x}_{a}-\widehat{x}\right)^{2}+\left(\boldsymbol{x}_{M(a)}-\widehat{x}\right)^{2} \leq \frac{2 r}{s} \sum_{u \in V_{K}}\left(\boldsymbol{x}_{u}-\widehat{x}\right)^{2} .
$$

As the definition of the projection operator $\boldsymbol{I}_{\perp \overrightarrow{1}}$ gives

$$
\boldsymbol{x}^{\top} \boldsymbol{I}_{\perp \overrightarrow{1}} \boldsymbol{x}=\min _{\widehat{x}} \sum_{u}\left(\boldsymbol{x}_{u}-\widehat{x}\right)^{2}
$$

we get

$$
\frac{r}{s} \boldsymbol{x}^{\top} \boldsymbol{L}_{M} \boldsymbol{x} \leq \frac{2 r}{s} \boldsymbol{x}^{\top} \boldsymbol{I}_{\perp \overrightarrow{1}} \boldsymbol{x}
$$

and incorporating the condition of $s \geq 4 r \varepsilon^{-1} / d$ gives a bound of

$$
\frac{r}{s} \boldsymbol{L}_{M} \preceq \frac{\varepsilon d}{2} \boldsymbol{I}_{\perp \overrightarrow{1}} .
$$

As the size $r$ biclique itself is a sum of $r$ matchings, we can apply a similar bound

$$
\boldsymbol{x}^{\top} \boldsymbol{L}_{K} \boldsymbol{x} \leq 2 r \sum_{u}\left(\boldsymbol{x}_{u}-\widehat{x}\right)^{2}
$$

for any scalar $\widehat{x}$, or equivalently,

$$
\frac{1}{s} \boldsymbol{L}_{K} \preceq \frac{\varepsilon d}{2} \boldsymbol{I}_{\perp \overrightarrow{1}} .
$$

Thus by triangle inequality, we can bound the mean zero random variable corresponding to the deviation incurred by matching $M_{i}$ by

$$
\left|\frac{r}{s} \boldsymbol{x}^{\top} \boldsymbol{L}_{M_{i}} \boldsymbol{x}-\frac{1}{s} \boldsymbol{x}^{\top} \boldsymbol{L}_{K} \boldsymbol{x}\right| \leq \varepsilon d \sum_{u}\left(\boldsymbol{x}_{u}-\widehat{x}\right)^{2}
$$


for any scalar $\widehat{x}$.

This gives the first condition on the magnitude of each random variable required by Bernstein's inequality as stated in Lemma 6.3. Specifically, summing over $M_{i}$, the random variable corresponding to $K$ is $\frac{r}{s}\left(\sum_{i=1}^{s} \boldsymbol{x}^{\top} \boldsymbol{L}_{M_{i}} \boldsymbol{x}\right)-\boldsymbol{x}^{\top} \boldsymbol{L}_{K} \boldsymbol{x}$. Since the expectation for any clique is 0, Lemma 7.3 gives that the variance per clique is at most

$$
\sum_{a \in V_{A}, b \in V_{B}} \frac{r}{s}\left(\boldsymbol{x}_{a}-\widehat{x}\right)^{2}\left(\boldsymbol{x}_{b}-\widehat{x}\right)^{2} .
$$

Furthermore, each vertex in $V_{S}$ can participate in at most $d / r$ such bicliques, as each of them incurs a degree of $r$. Since each edge has at least one endpoint in $V_{S}$, each edge occurs in at most $d / r$ bicliques, and the total variance is at most

$$
\sum_{u, v} \frac{d}{r} \cdot \frac{r}{s} \cdot\left(\boldsymbol{x}_{u}-\widehat{x}\right)^{2}\left(\boldsymbol{x}_{v}-\widehat{x}\right)^{2}=\left(\sqrt{d / s} \sum_{u}\left(\boldsymbol{x}_{u}-\widehat{x}\right)^{2}\right)^{2} \leq\left(\varepsilon d \cdot \sum_{u}\left(\boldsymbol{x}_{u}-\widehat{x}\right)^{2}\right)^{2}
$$

where we use $s \cdot d \geq \varepsilon^{-1 / 2} \cdot \varepsilon^{-3 / 2}=\varepsilon^{-2}$. The bound on deviation then follows from Bernstein's inequality as stated in Lemma 6.3.

Note that $s \geq 4 r \varepsilon^{-1} / d$ was required only to lower bound the probability that any edge was included in $H$, which in turn is needed to show that $H$ is a $\sqrt{\varepsilon}$ spectral sparsifier with high probability, something we will do in Section 7.2. This sparsifier property is required so that the guarantees in Section 6.3 apply, and we will use these guarantees later on, also in Section 7.2. Note that we could have enforced a tighter constraint of $s=\max \left(\varepsilon^{-1 / 2}, \frac{r \varepsilon^{-3 / 2}}{d}\right)$ and obtained a bound of $O\left(n \varepsilon^{-3 / 2}+k r \varepsilon^{-1 / 2}\right)$ edges in $H$, which would allow us to carry the rest of the proof forward. If we do this, $H$ will be a $\varepsilon^{3 / 4}$ spectral sparsifier (proof omitted). However, this ultimately does not improve our final bound on the edge count in a resistance sparsifier, so we have not set $s$ accordingly.

Lemma 7.4 guarantees that for a collection of balanced bicliques of equal size, where one side of the biclique has uniformly low degree in the original graph, that the error incurred by sampling matchings from that biclique is bounded in terms of that uniformly low degree.

We can then extend this procedure to general unit weighted bicliques and degree sequences. The idea is to break the bicliques of $\mathcal{K}_{B}$ up into a collection of balanced bicliques $\mathcal{K}_{B,=}$, where each edge $u v$ in the biclique has roughly the same value for $\min \left(\boldsymbol{d}_{u}, \boldsymbol{d}_{v}\right)$ where $\boldsymbol{d}_{u}$ represents the degree of $u$ in $\mathcal{K}_{B}$. This is done so that Lemma 7.4 can directly be applied to bound the variance of $\mathcal{K}_{B,=}$, as Lemma 7.4 provides good bounds on the variance in terms of the min-degree of an endpoint for any given edge in $\mathcal{K}_{B}$. The rough overview of the process is:

1. Incorporate vertices of low degree into $H$. This is done since the assumption on Lemma 7.4 is that $d \geq \varepsilon^{-1.5}$.

2. For each power of 2 , split up the bicliques as follows:

(a) For each biclique, find the set of edges with min-degree $2^{j-1} \leq d<2^{j}$. This will be the disjoint union of two bicliques.

(b) Balance the bicliques using MakeBALAnCED. 
(c) On the resulting bicliques: sparsify using SAmpleMatchings, with the appropriate value of $s$ as specified in Lemma 7.4 ,

The pseudocode of this routine is given in Algorithm 8, It transforms a collection of bicliques into a slightly larger (in total vertex count) collection of bicliques that are balanced, and which have the property that one side of the biclique has small degrees (in the original graph). This then allows us to invoke SampleMatchings on these bicliques with $d$ set to these degrees.

We first break the vertices based on their degrees, decompose a biclique into balanced bicliques. The formal guarantees of this step is:

Lemma 7.5. There is a routine $\operatorname{MakeBalanced}(G)$ that breaks any biclique on $n$ vertices into a sum of balanced bicliques with $2^{i}$ vertices whose total vertex count is $O(n \log n)$.

Proof. This decomposition has two steps: the first is to decompose $G$ into bicliques whose vertex sizes are powers of 2 , and the second is to make them balanced.

For the first step, suppose the two sides have $n_{1}$ and $n_{2}$ vertices respectively. We can write $n_{1}$ and $n_{2}$ both as sums of powers of 2 , and split the vertices into groups correspondingly. We create one biclique between each pair of powers of 2 present in $n_{1}$ and $n_{2}$. Since there are only $O(\log n)$ such groups on one side, one vertex can appear in $O(\log n)$ new bicliques. This increases the total number of vertices by a factor of $O(\log n)$.

Then in order to balance the bicliques, note that a $2^{i} \times 2^{j}$ biclique with $i<j$ is the sum of $2^{j-i}$ balanced bicliques with $2^{i}$ vertices on each side. This at most doubles the number of vertices, so overall we still have $O(n \log n)$ vertices.

With this guarantee, we can now prove the overall guarantees for an arbitrary degree sequence. Pseudocode of the algorithm is in Algorithm 8. Note that we do not need to pick a good bipartition as we're already working with bicliques.

Lemma 7.6. Let $\mathcal{K}_{B}$ be a collection of unit weighted bicliques on $n$ vertices whose sum $G\left(\mathcal{K}_{B}\right)$ has degree sequence $\boldsymbol{d}$. Then $\operatorname{SAmpleBiCliques}\left(\mathcal{K}_{B}, \varepsilon\right)$ returns in $\widetilde{O}\left(n \varepsilon^{-1.5}+n\left(\mathcal{K}_{B}\right) \varepsilon^{-0.5}\right)$ time, a graph $H$ sampled from a sum of independent random graphs such that:

1. $\mathbb{E}[H]=G\left(\mathcal{K}_{B}\right)$;

2. any graph $\widehat{H}$ in the support of $H$ satisfies

$$
\boldsymbol{L}_{\widehat{H}} \preceq \varepsilon \boldsymbol{D}_{G\left(\mathcal{K}_{B}\right) \perp \overrightarrow{1}}
$$

where $\boldsymbol{D}_{G\left(\mathcal{K}_{B}\right) \perp \overrightarrow{1}}$ is the diagonal matrix projected against the all $1 \mathrm{~s}$ vector;

3. the number of edges in $H$ is bounded by

$$
\widetilde{O}\left(n \varepsilon^{-1.5}+n\left(\mathcal{K}_{B}\right) \varepsilon^{-0.5}\right) ;
$$

where the trailing term is the total number of vertices in this collection of cliques, times an overhead of $\varepsilon^{-0.5} \log ^{O(1)} n$;

4. for any vector $\boldsymbol{x}$ and any value $\widehat{x}$, we have w.h.p.

$$
\left|\boldsymbol{x}^{\top} \boldsymbol{L}_{G\left(\mathcal{K}_{B}\right)} \boldsymbol{x}-\boldsymbol{x}^{\top} \boldsymbol{L}_{H} \boldsymbol{x}\right| \leq O\left(\varepsilon \log ^{2} n\right) \cdot \sum_{u} \boldsymbol{d}_{u}\left(\boldsymbol{x}_{u}-\widehat{x}\right)^{2} .
$$




$\overline{\text { Algorithm } 8 \text { SAmpleBiCliques }\left(\mathcal{K}_{B}, \varepsilon\right)}$
Input: A collection of $k$ unit weighted bicliques $\mathcal{K}_{B}$.

Error tolerance $\varepsilon$.

Output: Sketch $H$.

1. Compute the degrees of $G\left(\mathcal{K}_{B}\right)$, let $\boldsymbol{d}$ be the degree sequence.

2. Initialize $H$ to be empty.

3. For each vertex $u$ with $\boldsymbol{d}_{u} \leq \varepsilon^{-1.5}$,

(a) Add all edges incident to $u$ explicitly to $H$.

(b) Remove $u$ from all the entries in $\mathcal{K}_{B}$.

4. Let $V_{j}$ be the vertices with degrees $\boldsymbol{d}_{u}$ in the range $\left[2^{j-1}, 2^{j}-1\right]$.

5. For each $V_{j}$

(a) Initialize $\mathcal{K}_{B}(j)$ to be empty.

(b) (Break up each biclique into two bicliques such that one side of the biclique has degrees between $2^{j-1}$ and $2^{j}$, and the other side has degrees higher than $2^{j-1}$ ). For each $K \in \mathcal{K}_{B}$

i. Let $K_{A}$ and $K_{B}$ denote the two sides of biclique $K$. Let

$$
\begin{aligned}
& S_{A}:=K_{A} \cap V_{j}, \\
& S_{B}:=K_{B} \cap V_{j}, \\
& T_{A}:=K_{A} \cap\left(V_{j} \cup V_{j+1} \cup \ldots\right), \\
& T_{B}:=K_{B} \cap\left(V_{j} \cup V_{j+1} \cup \ldots\right) .
\end{aligned}
$$

Pass $S_{A} \times T_{B}, S_{B} \times\left(T_{A} \backslash S_{A}\right)$ into to $\mathcal{K}_{B}(j)$ implicitly (as subsets of vertices). These bicliques we pass are exactly the edges $(u, v)$ of $G(\mathcal{K})$ with $2^{j-1} \leq \min \left(\boldsymbol{d}_{u}, \boldsymbol{d}_{v}\right)<2^{j}$.

(c) For each $K \in \mathcal{K}_{B}(j)$, run $\operatorname{MakeBalanced}(B)$, and add the resulting bicliques to $\left\{\mathcal{K}_{B, 1}(j), \mathcal{K}_{B, 2}(j), \mathcal{K}_{B, 4}(j), \mathcal{K}_{B, 8}(j) \ldots\right\}$. In this step, a balanced biclique with $r$ vertices on each side (for any $r$ ) is added only to $\mathcal{K}_{B, r}(j$ ). (Note that each biclique resulting from this has $r=2^{l}$ vertices on each side, and one side has degrees between $2^{j-1}$ and $2^{j}$ in the original graph while the other has degrees at least $2^{j-1}$.)

(d) (Bucket $\mathcal{K}_{B, r}(j)$ by $r$ and run SampleMatchings on the buckets.) For each $r=2^{l}$

i. If $r \leq \varepsilon^{-1 / 2}$

A. Add all edges from $\left\{\mathcal{K}_{B, r}(j)\right\}$ to $H$ explicitly.

ii. Else
A. Let $H_{j, l} \leftarrow$ SampleMatchings $\left(\mathcal{K}_{B, r}(j), \max \left\{\varepsilon^{-1 / 2}, 4 r \varepsilon^{-1} 2^{-j}\right\}\right)$.
B. $H \leftarrow H \cup H_{j, l}$.

6. Return $H$. 
Proof. We start from the claim about the concentration of $\boldsymbol{x}^{\top} L_{H} \boldsymbol{x}$. Let $u v$ be an edge in $\mathcal{K}_{B}$, and without loss of generality, let $\boldsymbol{d}_{u} \leq \boldsymbol{d}_{v}$. In SampleBiCliques, edge $u v$ is placed into $\mathcal{K}_{B}\left(\left\lfloor\log \boldsymbol{d}_{u}\right\rfloor\right)$, the bucket of cliques corresponding to $\boldsymbol{d}_{u}$ in Step $5(\mathrm{~b}) \mathrm{i}$.

Therefore, by Lemma 7.4, the output of the calls to SAmpleBiCliques, $H_{j, l}$ satisfies

$$
\mathbb{E}\left[H_{i, l}\right]=G\left(\mathcal{K}_{B, 2^{l}}\right)
$$

any graph $\widehat{H}$ in its support satisfies

$$
\boldsymbol{L}_{\widehat{H}} \preceq \varepsilon \cdot 2^{j} \cdot \boldsymbol{I}_{V\left(\mathcal{K}_{B, 2}(j)\right) \perp \overrightarrow{1}}
$$

where $\boldsymbol{I}_{V\left(\mathcal{K}_{B, 2^{l}}(j)\right) \perp \overrightarrow{1}}$ is the identity matrix on $V\left(\mathcal{K}_{B, 2^{l}}(j)\right)$ projected against the all ones vector; and for any $\boldsymbol{x}$ and $\widehat{x}$, we have with high probability:

$$
\left|\boldsymbol{x}^{\top} \boldsymbol{L}_{H_{j, l}} \boldsymbol{x}-\boldsymbol{x}^{\top} \boldsymbol{L}_{\mathcal{K}_{B, l^{l}}(j)} \boldsymbol{x}\right| \leq O(\log n) \cdot \varepsilon \cdot \sum_{u \in V\left(\mathcal{K}_{B, 2^{l}}(j)\right)} 2^{j}\left(\boldsymbol{x}_{u}-\widehat{x}\right)^{2} .
$$

The first condition with linearity of expectation implies $\mathbb{E}[H]=G\left(\mathcal{K}_{B}\right)$.

To convert the second and third condition to global ones encompassing all $H_{j, l}$ s, the The key point here is that our construction was designed to guarantee that $u$ is not contained in any $\mathcal{K}_{B}(j)$ with $2^{j-1}>\boldsymbol{d}_{u}$. For the second condition, this gives that all vertices in $\mathcal{K}_{B}(j)$ have degrees at least $2^{j-1}$, so

$$
2^{j} \cdot \boldsymbol{I}_{V\left(\mathcal{K}_{B, 2^{l}}(j)\right) \perp \overrightarrow{1}} \preceq \boldsymbol{D}_{V\left(\mathcal{K}_{B, 2^{l}}(j)\right) \perp \overrightarrow{1}} \preceq \boldsymbol{D}_{G\left(\mathcal{K}_{B}\right) \perp \overrightarrow{1}},
$$

which bounds the sizes of the samples against the overall degree sequences.

For the third condition, the containment condition formalizes to

$$
\sum_{j: u \in \mathcal{K}_{B}(j)} 2^{j} \leq \sum_{2^{j-1} \leq \boldsymbol{d}_{u}} 2^{j} \leq 4 \boldsymbol{d}_{u}
$$

which coupled with the fact that there are only $O(\log n)$ distinct values of $r=2^{l}$ gives:

$$
\left|\boldsymbol{x}^{\top} \boldsymbol{L}_{H_{j, l}} \boldsymbol{x}-\boldsymbol{x}^{\top} \boldsymbol{L}_{\mathcal{K}_{B, 2^{l}}(j)} \boldsymbol{x}\right| \leq O\left(\log ^{2} n \cdot \varepsilon \sum_{u} \boldsymbol{d}_{u}\left(\boldsymbol{x}_{u}-\widehat{x}\right)^{2}\right),
$$

which proves concentration of $\boldsymbol{x}^{\top} \boldsymbol{L}_{H} \boldsymbol{x}$.

Now we move to bound the number of edges in $H$. Directly applying Lemma 7.4 gives that the number of edges in $H$ is always bounded above by:

$$
\sum_{j} \sum_{l} O\left(n \varepsilon^{-1.5}+n\left(\mathcal{K}_{B, 2^{l}}(j)\right) \varepsilon^{-1 / 2}\right) .
$$

For the total number of vertices, total number of vertices in $K_{B}(j)$ summed across all $j$ is $O(n \log n)$, and the number of vertices in $K_{B, 2^{i}}(j)$ summed across all $i$ and $j$ is $O(n \log n)$ by Lemma 7.5, So the first term is bounded by $O\left(n \log ^{2} n\right)$. 
For the second term, $\sum_{j, l} n\left(\mathcal{K}_{j, l}\right)$ tracks the sum of vertex counts across all cliques in $K_{B, 2^{l}}(j)$ for any $j$ and $l$. Note that each clique in $K_{B, 2^{i}}(j)$ has a corresponding clique $K \in \mathcal{K}_{B}$ that it originated from and is a subgraph of. Edges from each clique in $K$ can go to at most $O\left(\log ^{2} n\right)$ different values of $j$ and $l$, so therefore

$$
\sum_{j, l} n\left(\mathcal{K}_{B, 2^{l}}(j)\right) \leq \sum_{K \in \mathcal{K}_{B}} O\left(\log ^{2} n\right) n(K) \leq O\left(\log ^{2} n\right) n(\mathcal{K})
$$

and we get our bound on edge count.

\subsection{Incorporating Recursive Expander Decompositions}

We now incorporate expander partitioning in a way analogous to Section 6.2. The main result that we shall prove in this section is:

Lemma 7.7. Given a collection of unit weighted bicliques $\mathcal{K}$, and any error $\varepsilon$, invoking the routine $\operatorname{ImplicitSketchUnweightedBiCliques}(\mathcal{K}, \widehat{\varepsilon}, \phi, q)$ as shown in Algorithm 10 with

$$
\begin{aligned}
& q=O\left(\sqrt{\frac{\log n}{\log \log n}}\right), \\
& \phi=n^{-2 / q}=\exp (-O(\sqrt{\log n \log \log n})), \\
& \widehat{\varepsilon}=\frac{\phi^{4}}{O(1) \cdot \log ^{4} n \cdot q} \varepsilon
\end{aligned}
$$

produces in time

$$
n^{1+o(1)} \varepsilon^{-3 / 2}+n(\mathcal{K}) n^{o(1)} \varepsilon^{-1 / 2}
$$

a graph $H$ such that:

1. $H$ has $n^{1+o(1)} \varepsilon^{-1.5}+n(\mathcal{K}) n^{o(1)} \varepsilon^{-0.5}$ edges.

2. With high probability, $H$ is a $\sqrt{\varepsilon}$-approximation to $G(\mathcal{K})$, i.e., $\boldsymbol{L}_{H} \approx_{\sqrt{\varepsilon}} \boldsymbol{L}_{G(\mathcal{K})}$.

3. For any fixed vector $\boldsymbol{x} \in \mathbb{R}^{V}$, with high probability we have $\boldsymbol{x}^{\top} \boldsymbol{L}_{G(\mathcal{K})} \boldsymbol{x} \approx_{\varepsilon} \boldsymbol{x}^{\top} \boldsymbol{L}_{H} \boldsymbol{x}$.

Note that the constants in the base of $\phi$, and in turn $q$, depends on the exponent of $\log n$ in $\gamma_{\mathrm{ST}}(n)$, the overhead from the expander decomposition routine given in Lemma 6.7.

Expander decomposition as stated in Lemma 6.7 works only on an explicitly specified graph. Our goal is to run in time almost-linear in the number of vertices of these bicliques, instead of the number of edges. Instead, we will compute such a decomposition on a spectral sparsifier of the sum of bicliques, and transfer the errors using the spectral guarantees. Specifically, we invoke the following fact which plays a central role in previous results on implicitly sparsifying graphs [PS14, $\left.\mathrm{KLP}^{+} 16, \mathrm{CKP}^{+} 17, \mathrm{CCL}^{+} 15\right]$ :

Lemma 7.8. There exists an absolute constant $C_{S}$ depending on the (w.h.p.) success probability such that for any balanced biclique $K_{B, r}$ with $r$ vertices on each side, the union of $s$ random matchings for $s=C_{S} \log n$,

$$
\widetilde{G}=\operatorname{SampleMatchings}\left(\left\{K_{B, r}\right\}, s\right)
$$


has the same (weighted) degrees as $K_{B, r}$, and satisfies with high probability:

$$
\boldsymbol{L}_{G\left(K_{B, r}\right)} \approx_{2} \boldsymbol{L}_{\widetilde{G}}
$$

Running this on each biclique reduces the number of edges to $O\left(n\left(\mathcal{K}_{B, r}\right) \log n\right)$, instead of edges, involved in the expander representation given by $\mathcal{K}_{B, r}$. This is small enough to allow us to explicitly run expander decompositions on this graph, which also has the same weights on each edge. Note that here we only invoke SAMPLEMATChings in the constant error regime, so do not need to perform a more careful analysis of its $\varepsilon$ dependencies. This approximation between $\widetilde{G}$ and $G(\mathcal{K})$ is useful in two ways:

1. Any partition of vertices cuts a similar weight of edges in the two graphs. This means the process of sparsifying the edges within clusters, and then repeating on edges between clusters makes sufficient progress to terminate in a few rounds.

2. The sum of $\boldsymbol{d}_{u}\left(\boldsymbol{x}_{u}-\widehat{x}\right)^{2}$ is the same in the two graphs. Combining this with the error guarantees of quadratic forms means that we can still transform the degree-dependent guarantees from Lemma 7.6 to guarantees involving the Laplacian quadratic form in a way analogous to the proof of Theorem 6.1 Part 2).

Note in particular for the second condition, it is not the case that an expander subgraph in a sparsifier $(\widetilde{G})$ is an expander in the original graph $(G(\mathcal{K}))$. Consider for example adding/removing an expander on a small subset of vertices that would be otherwise independent in a larger expander. Instead, we need to bound the overall costs in degrees against the quadratic form of the sparsifier, and transform the costs over via the overall quadratic form.

Note that in order to work with a unit weighted graph for expander partitioning, we need to restrict to balanced bicliques with size $r$ once more.

The pseudocode of the algorithm is divided into two parts: Algorithm 9 performs the partition, and sparsifies the edges within the clusters. The outer loop in Algorithm 10 then creates another representation by bicliques of the edges between the pieces, and recurses upon them.

Lemma 7.9. Given any collection of $k$ balanced bicliques $\mathcal{K}$, and any error $\varepsilon$ and $\phi$, ImplicitPartition AndSAmple $(\mathcal{K}, \varepsilon, \phi)$ returns in $\widetilde{O}\left(n \varepsilon^{-1.5}+n(\mathcal{K}) \varepsilon^{-0.5}+n(\mathcal{K}) \phi^{-2}\right)$ time a partition $\left\{\widehat{S}_{1}, \widehat{S}_{2}, \ldots\right\}$ and a graph $H$ such that with high probability:

1. $H$ has at most $\widetilde{O}\left(n \varepsilon^{-1.5}+n(\mathcal{K}) \varepsilon^{-0.5}\right)$ edges,

2. the number of edges in $\mathcal{K}$ on the boundary of some cluster, is at most $4 \gamma_{\mathrm{ST}}(n) \phi m(\mathcal{K})$, and if we let $G(\mathcal{K})\left[\widehat{S}_{i}\right]$ be the edges of $G(\mathcal{K})$ contained in some piece $\widehat{S}_{i}$, we have

1. With high probability

$$
\boldsymbol{L}_{H} \approx_{O\left(\sqrt{\phi^{-4} \varepsilon \log n}\right)} \sum_{i} \boldsymbol{L}_{G(\mathcal{K})\left[\widehat{S}_{i}\right]} .
$$

2. For any fixed vector $\boldsymbol{x}$, with high probability

$$
\left|\boldsymbol{x}^{\top} \boldsymbol{L}_{H} \boldsymbol{x}-\sum_{i} \boldsymbol{x}^{\top} \boldsymbol{L}_{G(\mathcal{K})\left[\widehat{S}_{i}\right]} \boldsymbol{x}\right| \leq O\left(\phi^{-4} \log ^{4} n\right) \cdot \varepsilon \cdot \boldsymbol{x}^{\top} \boldsymbol{L}_{\widetilde{G}(\mathcal{K})} \boldsymbol{x} .
$$


Algorithm 9 ImplicitPartitionAndSample $\left(\mathcal{K}_{B, r}, \varepsilon, \phi\right)$

Input: Collection of balanced bicliques $\mathcal{K}_{B, r}$. Error threshold $\varepsilon$. Conductance $\phi$.

Output: Partition $\left\{\widehat{S}_{1}, \widehat{S}_{2}, \ldots\right\}$ of the vertices, sketch $H$ of the edges of $\mathcal{K}_{B, r}$ contained in the partitions $\widehat{S}_{1}, \widehat{S}_{2}, \ldots$

1. Set $s=C_{S} \log n$, and build $\widetilde{G}=\sum_{K_{B, r} \in \mathcal{K}_{B, r}} \operatorname{SampleMatchings}\left(\left\{K_{B, r}\right\}, s\right)$ explicitly.

2. $\left\{\widehat{S}_{1}, \widehat{S}_{2}, \ldots\right\} \leftarrow \operatorname{ExpanderDeCOMpose}(\widetilde{G}, \phi)$.

3. For each $i$

(a) Initialize $\mathcal{K}_{i}$ to empty

4. For each $K_{B, r} \in \mathcal{K}_{B, r}$ and each $\widehat{S}_{i}$ with non-zero intersection with it

(a) $\mathcal{K}_{i} \leftarrow \mathcal{K}_{i} \cup K_{B, r}\left[\widehat{S}_{i}\right]$, where $K_{B, r}\left[\widehat{S}_{i}\right]$ is the portion of $K_{B, r}$ contained in $\widehat{S}_{i}$.

5. Initialize $H$ to empty.

6. For each $\mathcal{K}_{i}$

(a) $H_{i} \leftarrow \operatorname{SampleBiCliques}\left(\mathcal{K}_{i}, \varepsilon\right)$

(b) $H \leftarrow H \cup H_{i}$

7. Return $\left\{\widehat{S}_{1}, \widehat{S}_{2}, \ldots\right\}, H$.

Proof. Applying the guarantees of Lemma 7.8 to each $K_{B, r} \in \mathcal{K}_{B, r}$ and summing over the results gives that the crude sparsifier generated on Line 1 satisfies:

$$
\widetilde{G} \approx_{2} G\left(\mathcal{K}_{B, r}\right)
$$

and the degrees of $\widetilde{G}$ and $G\left(\mathcal{K}_{B, r}\right)$ are the same.

We first bound the edge count between clusters. For the edges in $H_{i}$, the result follows from the clusters $\widehat{S}_{i}$ being disjoint and Lemma 7.6.

For the edges between clusters, the guarantees of expander decomposition gives that the number of such edges in $\widetilde{G}$, when treated as a unit graph, is $\gamma_{\mathrm{ST}}(n) \phi|E(\widetilde{G})|$. Since each of these edges has weight $r / s$, and both the total number of edges, and edges across cuts, are within factors of 2 in $G(\mathcal{K})$ and $\widetilde{G}$, the total number of edges on the boundaries of some $\widehat{S}_{i}$ in $G$ is at most

$$
4 \gamma_{\mathrm{ST}}(n) \phi|E(G(\mathcal{K}))|=4 \gamma_{\mathrm{ST}}(n) \phi m(\mathcal{K}) .
$$

We now give the approximation guarantees. The guarantees of expander decompositions from Lemma 6.7 gives that each $\widehat{S}_{i}$ is contained in some $S_{i}$ (which are unknown to the algorithm) such that $G\left[S_{i}\right]$ has conductance at least $\phi^{2}$. Cheeger's inequality from Lemma 6.5 then gives

$$
\boldsymbol{L}_{G\left[S_{i}\right]} \succeq \frac{\phi^{4}}{2} \boldsymbol{D}_{S_{i}, \perp 1} \succeq \frac{\phi^{4}}{2} \boldsymbol{D}_{\widehat{S}_{i}, \perp 1},
$$

This, combined with Claim 2 of Lemma 7.6 means that any graph $\widehat{H}$ sampled in the creation of $H_{i}$ is small w.r.t. $G\left(\mathcal{K}_{B, r}\right)$ :

$$
\boldsymbol{L}_{\widehat{H}} \preceq \varepsilon \boldsymbol{D}_{\widehat{S}_{i}, \perp 1} \preceq 2 \varepsilon \phi^{-4} \boldsymbol{L}_{G\left[S_{i}\right]} \preceq 2 \varepsilon \phi^{-4} \boldsymbol{L}_{G} .
$$


So by Lemma 7.2 , gives

$$
\boldsymbol{L}_{H_{i}} \approx_{O\left(\sqrt{\varepsilon \phi^{-4} \log n}\right)} \boldsymbol{L}_{G(\mathcal{K})\left[\widehat{S}_{i}\right]}
$$

which, as the sampled graphs are disjoint, gives the approximation between $G$ and $H$.

We now turn to the per-vector guarantee. Lemma 7.6 gives that on each partition $\widehat{S}_{i}$, we have for any $\widehat{x}$

$$
\left|\boldsymbol{x}^{\top} \boldsymbol{L}_{G(\mathcal{K})\left[\widehat{S}_{i}\right]} \boldsymbol{x}-\boldsymbol{x}^{\top} \boldsymbol{L}_{H_{i}} \boldsymbol{x}\right| \leq O\left(\log ^{2} n\right) \cdot \varepsilon \cdot \sum_{u \in \widehat{S}_{i}} \boldsymbol{d}_{u}\left(\boldsymbol{x}_{u}-\widehat{x}\right)^{2} \leq O\left(\log ^{2} n\right) \cdot \varepsilon \cdot \sum_{u \in S_{i}} \boldsymbol{d}_{u}\left(\boldsymbol{x}_{u}-\widehat{x}\right)^{2} .
$$

As $\widetilde{G}$ and $G\left(\mathcal{K}_{B, r}\right)$ have the same degree sequences, we can make the critical step of interpreting the last term on $\widetilde{G}$. Lemma 6.6 and the guarantees of expander decompositions from Lemma 6.7 then implies that for appropriate choices of $\widehat{x}$ per expander cluster $S_{i}$, specifically

$$
\widehat{x}_{i}:=\frac{\sum_{u \in S_{i}} \boldsymbol{d}_{u} \boldsymbol{x}_{u}}{\sum_{u \in S_{i}} \boldsymbol{d}_{u}}
$$

gives

$$
\sum_{u \in S_{i}} \boldsymbol{d}_{u}\left(\boldsymbol{x}_{u}-\widehat{x}\right)^{2} \leq O\left(\phi^{-4} \log n\right) \boldsymbol{x}^{\top} \boldsymbol{L}_{\widetilde{G}\left[S_{i}\right]} \boldsymbol{x}
$$

or if we sum over all partitions $\widehat{S}_{i}$ gives

$$
\left|\sum_{i} \boldsymbol{x}^{\top} \boldsymbol{L}_{G(\mathcal{K})\left[\widehat{S}_{i}\right]} \boldsymbol{x}-\boldsymbol{x}^{\top} \boldsymbol{L}_{H} \boldsymbol{x}\right|=\sum_{i}\left|\boldsymbol{x}^{\top} \boldsymbol{L}_{G(\mathcal{K})\left[\widehat{S}_{i}\right]} \boldsymbol{x}-\boldsymbol{x}^{\top} \boldsymbol{L}_{H_{i}} \boldsymbol{x}\right| \leq O\left(\phi^{-4} \log ^{3} n\right) \cdot \varepsilon \cdot \sum_{i} \boldsymbol{x}^{\top} \boldsymbol{L}_{\widetilde{G}\left[S_{i}\right]} \boldsymbol{x} .
$$

We can then invoke the condition on the at most $O(\log n)$ total overlaps between the $S_{i}$ s from Part $2 \mathrm{~b}$ of Lemma 6.7 to get

$$
\sum_{i} \boldsymbol{L}_{\widetilde{G}\left[S_{i}\right]} \preceq O(\log n) \boldsymbol{L}_{\widetilde{G}}
$$

and in turn

$$
\left|\sum_{i} \boldsymbol{x}^{\top} \boldsymbol{L}_{G(\mathcal{K})\left[\widehat{S}_{i}\right]} \boldsymbol{x}-\boldsymbol{x}^{\top} \boldsymbol{L}_{H} \boldsymbol{x}\right| \leq O\left(\varepsilon \phi^{-4} \log ^{4} n\right) \boldsymbol{x}^{\top} \boldsymbol{L}_{\widetilde{G}} \boldsymbol{x} \leq O\left(\varepsilon \phi^{-4} \log ^{4} n\right) \boldsymbol{x}^{\top} \boldsymbol{L}_{G\left(\mathcal{K}_{B, r}\right)} \boldsymbol{x} .
$$

where the last inequality again follows from the spectral approximations between $G\left(\mathcal{K}_{B, r}\right)$ and $\widetilde{G}$.

The time bound holds because we run ExPANDERDECOMPOSE on a graph with $O(n(\mathcal{K}) \log n)$ edges with parameter $\phi$, and we run SAmpleBiCliques on $\mathcal{K}_{i}$ for all $i$, where the sum of the vertices in $\mathcal{K}_{i}$ across all $i$ is bounded above by $n(\mathcal{K})$. Note that the number of vertices in each $\widehat{S}_{i}$ across all $i$ is equal to $n$. Therefore, the total runtime (by invoking the runtimes in Lemma 6.7 and Lemma 7.6 respectively) gives a total runtime of:

$$
\widetilde{O}\left(n(\mathcal{K}) \phi^{-2}+n \varepsilon^{-1.5}+n(\mathcal{K}) \varepsilon^{-0.5}\right)
$$

as desired. 
We then need to repeat our process on the edges between the pieces $\widehat{S}_{i}$. Note again that explicitly writing down these edges would incur a significantly higher cost. So our recursion relies on the interaction between a biclique and a partition of vertices, which we will show is also a sum of bicliques whose total representation size (in terms of vertex counts) is only higher by a factor of $O(\log n)$.

Lemma 7.10. Let $K$ be a biclique on $n$ vertices, and $\widehat{S}_{1}, \widehat{S}_{2}, \ldots$ be a partition of $i t s$ vertices. Then

1. for any $i$, the graph $K\left[\widehat{S}_{i}\right]$, that's the restriction of $K$ to $\widehat{S}_{i}$, is also a biclique, and

2. the edges between the pieces,

$$
\bigcup_{1 \leq i \leq t} E_{K}\left[\widehat{S}_{i}, V \backslash \widehat{S}_{i}\right]
$$

is also the union of bicliques whose total vertex count is $O(n \log n)$.

Proof. The restriction of $K$ to $\widehat{S}_{i}$ is a biclique between the subsets of both partitions contained in $\widehat{S}_{i}$. The boundary edges can be dealt with via a divide-and-conquer argument:

1. If any $\widehat{S}_{i}$ has more than $1 / 3$ of the vertices, its boundary is the sum of two bicliques to $V \backslash \widehat{S}_{i}$, and we can recurse on at most $(2 / 3) n$ vertices in $V \backslash \widehat{S}_{i}$.

2. Otherwise we can partition the $\widehat{S}_{i}$ s into two subsets, each with at most $(2 / 3) n$ vertices. The edges between these two subsets is once again the sum of two bicliques, and the overall decomposition follows from recursing on the two halves.

In each case, the size of the subproblems that we recurse on decreases by a constant factor, and they have at most $n$ vertices. This means there are at most $O(\log n)$ layers of recursion, for a total of $O(n \log n)$ vertices in these bicliques.

This means we can then recurse on the boundary edges, leading to an overall recursive scheme whose pseudocode is in Algorithm 10.

Proof. (of Lemma 7.7) We start with the approximation guarantees. This follows from noting that each $\mathcal{K}_{j}^{n e x t}$ are edge-disjoint, so the total error across each layer of the recursion sums to at most

$$
O\left(\phi^{-4} \log ^{4} n\right) \cdot \widehat{\varepsilon} \cdot \boldsymbol{x}^{\top} \boldsymbol{L}_{G(\mathcal{K})} \boldsymbol{x} \leq \frac{\epsilon}{O(q)} \boldsymbol{x}^{\top} \boldsymbol{L}_{G(\mathcal{K})} \boldsymbol{x},
$$

which summed across the $q$ levels of recursion gives $\left|\boldsymbol{x}^{\top} \boldsymbol{L}_{H} \boldsymbol{x}-\boldsymbol{x}^{\top} \boldsymbol{L}_{G(\mathcal{K})} \boldsymbol{x}\right| \leq 0.1 \epsilon \cdot \boldsymbol{x}^{\top} \boldsymbol{L}_{G(\mathcal{K})} \boldsymbol{x}$, or $\boldsymbol{x}^{\top} \boldsymbol{L}_{G(\mathcal{K})} \boldsymbol{x} \approx_{0.1 \varepsilon} \boldsymbol{x}^{\top} \boldsymbol{L}_{H} \boldsymbol{x}$.

We now bound the size of the output and overall running time. Lemma 7.9 gives that the edges between the pieces is bounded by

$$
4 \gamma_{\mathrm{ST}}(n) \phi \cdot m(\mathcal{K})
$$

where $\gamma_{\mathrm{ST}}(n)$ is the polylog overhead from expander decomposition from Lemma 6.7. This means that after $q$ levels of recursion, the total number of edges in the bicliques between the pieces is at most

$$
\left(4 \gamma_{\mathrm{ST}}(n) \phi\right)^{q} \cdot m(\mathcal{K}) \leq\left(4 \gamma_{\mathrm{ST}}(n) \phi\right)^{q} \cdot n^{2}
$$


Algorithm 10 ImplicitSketchUnweightedBiCliques $\left(\mathcal{K}_{B}, \varepsilon, \phi, q\right)$

Input: Collection of bicliques $\mathcal{K}_{B}$, Error threshold $\varepsilon$. Conductance $\phi$ and recursion layer $q$.

Output: sketch $H$.

1. If $q=0$

(a) Let $H$ be the sum of all edges in each $G_{i}$ explicitly.

2. Else

(a) Initialize $H$ as empty.

(b) $\left\{\mathcal{K}_{B, 1}, \mathcal{K}_{B, 2}, \mathcal{K}_{B, 4} \cdots\right\} \leftarrow$ MakeBalanced $\left(\mathcal{K}_{B}\right)$.

(c) For each $r=2^{l}$

i. $\left\{\widehat{S}_{1}, \widehat{S}_{2}, \ldots\right\}, H_{l} \leftarrow$ ImplicitPartitionAndSAmple $\left(\mathcal{K}_{B, r}, \varepsilon, \phi\right)$.

ii. $H \leftarrow H \cup H_{l}$.

iii. Let $\mathcal{K}_{B, r}^{\text {next }}$ be the intersection of $\mathcal{K}_{B, r}$ with the boundary of the partitions $\left\{\widehat{S}_{1}, \widehat{S}_{2}, \ldots\right\}$, implicitly represented using Lemma 7.10 .

iv. $H \leftarrow H \cup \operatorname{Implicit} \operatorname{SketchUnweightedBiCliques}\left(\mathcal{K}_{B, r}^{n e x t}, \varepsilon, \phi, q-1\right)$.

3. Return $H$.

On the other hand, Lemma 7.10 gives that the number of vertices in the bicliques increases by a factor of $c_{1} \log n$ per iteration, leading to a total edge count of

$$
q n \varepsilon^{-1.5}+n(\mathcal{K})\left(c_{1} \log n\right)^{q} \varepsilon^{-0.5}+\left(4 \gamma_{\mathrm{ST}}(n) \phi\right)^{q} \cdot m,
$$

where the last term comes from explicitly forming the graph once $q$ reaches 0 in the recursion. The choices of

$$
\begin{aligned}
& q=\exp \left(O\left(\sqrt{\frac{\log n}{\log \log n}}\right)\right) \\
& \phi=\exp (-O(\sqrt{\log n \log \log n})),
\end{aligned}
$$

bounds the overhead on $n(\mathcal{K}) \epsilon^{-0.5}$ :

$$
\left(c_{1} \log n\right)^{q}=\exp \left(O(\log \log n) \cdot O\left(\sqrt{\frac{\log n}{\log \log n}}\right)\right) \leq \exp (O(\sqrt{\log n \log \log n})) \leq n^{o(1)}
$$

and the fraction of edges remaining:

$$
\left(4 \gamma_{\mathrm{ST}}(n) \phi\right)^{q} \leq \exp (-O(\sqrt{\log n \log \log n}) \cdot q) \leq \exp (-O(\log n)) \leq m^{-1} .
$$

The choices of the two parameters also result in the running time of the steps being almost-linear in the representation size of $\mathcal{K}, n(\mathcal{K})$. Note that the allowed error, $\widehat{\varepsilon}$ also needs to be adjusted to account for the $q$ steps, as well as the overhead from Lemma 7.9 . 


\subsection{From Square-Sparsifiers to Resistances}

Now that we can efficiently sketch bicliques, it only remains to show how this interacts with being able to compute resistance estimates in a graph. This top-most level of the algorithm relies on several reductions. The purpose of this section is to elaborate on those reductions, and reference prior works from which they are largely obtained.

The first of these is Section 6 of $\left[\mathrm{DKP}^{+} 17\right]$, which is a reduction (via recursion) from computing Schur complements to computing effective resistances. The key definition is the Schur complement of a graph Laplacian:

Definition 7.11. The Schur complement of a graph Laplacian $\boldsymbol{L}$ onto a subset of vertices $C$, $\mathrm{SC}(\boldsymbol{L}, C)$, is obtained by splitting the matrix $\boldsymbol{L}$ into blocks separating $C$ and $F=V \backslash C$ :

$$
\boldsymbol{L}=\left[\begin{array}{ll}
\boldsymbol{L}_{F F} & \boldsymbol{L}_{F C} \\
\boldsymbol{L}_{C F} & \boldsymbol{L}_{C C}
\end{array}\right]
$$

and then computing the $C \times C$ matrix

$$
\operatorname{SC}(\boldsymbol{L}, C):=\boldsymbol{L}_{C C}-\boldsymbol{L}_{C F} \boldsymbol{L}_{F F}^{+} \boldsymbol{L}_{F C} .
$$

The Schur complement has a natural interpretation as the intermediate state of Gaussian elimination after removing $F$. However, for algorithmic purposes, the above closed form is arguably more important $\left[\mathrm{KLP}^{+} 16, \mathrm{KS}_{16}, \mathrm{DKP}^{+} 17, \mathrm{DPPR} 17\right]$. This is due to it exactly preserving a variety of quantities, especially effective resistances.

Fact 7.12. For any pair of vertices $u, v \in C$, the effective resistance between $u$ and $v$ is the same in $\mathrm{SC}(\boldsymbol{L}, C)$ and $\boldsymbol{L}$.

We remark that this preservation of resistances is due to the more general fact that the inverse of the Schur complement, $\mathrm{SC}(\boldsymbol{L}, C)^{+}$is the minor on $C$ of the pseudo-inverse of $\boldsymbol{L},\left(\boldsymbol{L}^{+}\right)_{C, C}$, after projection against the null space. However, for the purpose of estimating effective resistances, the interaction with algorithms for producing approximate Schur complements can be summarized as follows.

Lemma 7.13. (Reduction from Computing Effective Resistances to Resistance-Approximating Sketches of Schur Complements, Section 6 of $\left.\left.\left[D K P^{+}{ }^{1}\right\}\right]\right)$ Given a routine that takes a graph on $\widehat{n}$ vertices, $\widehat{m}$ edges, a subset of vertices $C$, and an error parameter $\widehat{\varepsilon}$, and returns a resistance-preserving sketch of $\operatorname{SC}(\boldsymbol{L}, C)$ of size $\widehat{n} \Delta(\widehat{n}, \widehat{\varepsilon})$ in time $T_{S C}(\widehat{n}, \widehat{m}, \widehat{\varepsilon})$, we can compute $1 \pm \epsilon$ approximations of resistances between $t$ pairs of vertices of a graph on $n$ vertices and $m$ edges in time

$$
\widetilde{O}\left(T_{S C}\left(n, m, \frac{\epsilon}{2}\right)+\left(1+\frac{t}{n}\right) \cdot T_{S C}\left(n, n \Delta\left(n, \frac{\varepsilon}{O(\log n)}\right), \frac{\varepsilon}{O(\log n)}\right)\right) .
$$

So our goal becomes adapting the resistance sparsifiers from Section 6 to work (implicitly) on Schur complements. However, the lack of matrix concentration/martingale bounds precludes us from invoking the single-vertex pivoting schemes from [KS16]. Instead, we utilize the square and

\footnotetext{
${ }^{8}$ version 1 at https://arxiv.org/pdf/1611.07451v1.pdf
} 
sparsify method from Appendix G of $\left[\mathrm{KLP}^{+} 16\right]$, which has a higher polylog overhead, but yields more control over the intermediate states. Specifically, for a block decomposition into $F$ and $C$ with the top-left block further decomposed into

$$
\boldsymbol{L}_{F F}=\boldsymbol{D}_{F F}-\boldsymbol{A}_{F F},
$$

the Schur complement onto $C$ can be expressed as

$$
\operatorname{SC}(\boldsymbol{L}, C)=\frac{1}{2} \operatorname{SC}\left(\left[\begin{array}{cc}
\boldsymbol{L}_{F F}-\boldsymbol{A}_{F F} \boldsymbol{D}_{F F}^{-1} \boldsymbol{A}_{F F} & \boldsymbol{L}_{F C}+\boldsymbol{A}_{F F} \boldsymbol{D}_{F F}^{-1} \boldsymbol{L}_{F C} \\
\boldsymbol{L}_{C F}+\boldsymbol{L}_{C F} \boldsymbol{D}_{F F}^{-1} \boldsymbol{A}_{F F} & 2 \boldsymbol{L}_{C C}-\boldsymbol{L}_{C F} \boldsymbol{D}_{F F}^{-1} \boldsymbol{L}_{F C}
\end{array}\right]\right)
$$

This is given in Equation 10 of $\left[\mathrm{KLP}^{+} 16\right]$. Note that $\boldsymbol{L}_{C F}$ consists of entirely off-diagonal entries, and is often also represented as $-\boldsymbol{A}_{C F}$, a minor of the adjacency matrix, instead.

Somewhat surprisingly, this matrix is in fact a graph Laplacian. Furthermore, it decomposes into sums of weighted cliques and weighted bipartite cliques. Formally a weighted (bi)clique is given by a set of vertex weights $\boldsymbol{w}_{u}$, with the edge between $u$ and $v$ having weight $\boldsymbol{w}_{u} \cdot \boldsymbol{w}_{v}$. Here, the terms on the top-left and bottom-right blocks, $\boldsymbol{A}_{F F} \boldsymbol{D}_{F F}^{-1} \boldsymbol{A}_{F F}$ and $\boldsymbol{L}_{C F} \boldsymbol{D}_{F F}^{-1} \boldsymbol{L}_{F C}$ are sums of weighted cliques on $F$ and $C$, and $-\boldsymbol{A}_{F F} \boldsymbol{D}_{F F}^{-1} \boldsymbol{L}_{F C}$ is a sum of weighted bicliques between $F$ and C. Specifically,

- $\boldsymbol{A}_{F F} \boldsymbol{D}_{F F}^{-1} \boldsymbol{A}_{F F}$ is a sum of cliques on $F$, one per vertex of $f \in C$. The weight of an edge between $f_{1}$ and $f_{2}$ in the clique centered at $f$ is given by

$$
\frac{\boldsymbol{A}_{f_{1} f} \boldsymbol{A}_{f_{2} f}}{\boldsymbol{D}_{f f}},
$$

which corresponds to a weight vector $\boldsymbol{w}_{\widehat{f}}=\boldsymbol{D}_{f f}^{-1 / 2} \boldsymbol{A}_{\widehat{f} f}$.

- $\boldsymbol{L}_{C F} \boldsymbol{D}_{F F}^{-1} \boldsymbol{L}_{F C}$ is a sum of cliques on $C$, one per vertex of $f \in F$. The weight of an edge between $c_{1}$ and $c_{2}$ in the clique centered at $f$ is given by

$$
\frac{\boldsymbol{L}_{c_{1} f} \boldsymbol{L}_{c_{2} f}}{\boldsymbol{D}_{f f}}
$$

which corresponds to a weight of $\boldsymbol{w}_{\widehat{c}}=-\boldsymbol{D}_{f f}^{-1 / 2} \boldsymbol{L}_{\widehat{c} f}$. Note that the negation is necessary because the off-diagonal entries of $\boldsymbol{L}$ are negative.

- $-\boldsymbol{A}_{F F} \boldsymbol{D}_{F F}^{-1} \boldsymbol{L}_{F C}$ is a sum of bicliques between $F$ and $C$, one per vertex in $F$. The weight of an edge between $\widehat{c}$ and $\widehat{f}$ in the clique centered at $f$ is given by

$$
\frac{-\boldsymbol{A}_{\widehat{c f}} \boldsymbol{L}_{\widehat{f} f}}{\boldsymbol{D}_{f f}},
$$

which, as off-diagonal entries of $\boldsymbol{L}$ are negative, corresponds to a weight of

$$
\begin{aligned}
& \boldsymbol{w}_{\widehat{f}}=\boldsymbol{D}_{f f}^{-1 / 2} \boldsymbol{A}_{\widehat{f} f}, \\
& \boldsymbol{w}_{\widehat{c}}=-\boldsymbol{D}_{f f}^{-1 / 2} \boldsymbol{L}_{\widehat{c} f} .
\end{aligned}
$$

\footnotetext{
${ }^{9}$ we cite version 1 at https://arxiv.org/pdf/1512.01892v1.pdf
} 
This provides the interaction point with the clique sparsifiers from Lemma 7.7. However, as Lemma 7.7 only works for a sum of unweighted bicliques, we need to make two additional transformations.

Lemma 7.14. Any weighted clique on $n$ vertices is equivalent to a sum of weighted bicliques on a total of $O(n \log n)$ vertices.

Proof. This is via divide-and-conquer: we can split the vertices into two halves of (roughly) equal size, and represent all edges between them by a biclique. Then recursing on both halves gives a total of $O(n \log n)$ vertices.

Lemma 7.15. A weighted biclique on $n$ vertices with weights in a poly $(n)$ range can be approximated by a sum of uniform weighted bicliques on a total of $O\left(n \log ^{2} n\right)$ vertices while incurring a multiplicative error of $1 / \operatorname{poly}(n)$.

Proof. We first decompose each $\boldsymbol{w}_{u}$ into its binary representation. Here we can truncate after $O(\log n)$ bits while incurring an error of $1 / \operatorname{poly}(n)$.

Then an edge of weight $\boldsymbol{w}_{u} \cdot \boldsymbol{w}_{v}$ can be factored based on the binary representation of $\boldsymbol{w}_{u}$ and $\boldsymbol{w}_{v}$. Suppose the 1 bits in the binary representation of $\boldsymbol{w}_{u}$ are $i_{u, 1}, i_{u, 2} \ldots i_{u, l(u)}$, and similarly for $v$, then we have

$$
\begin{aligned}
& \boldsymbol{w}_{u}=2^{i_{u, 1}}+2^{i_{u, 2}}+\ldots+2^{i_{u, l(u)}} \\
& \boldsymbol{w}_{v}=2^{i_{v, 1}}+2^{i_{v, 2}}+\ldots+2^{i_{v, l(v)}}
\end{aligned}
$$

which then gives

$$
\boldsymbol{w}_{u} \cdot \boldsymbol{w}_{v}=\prod_{1 \leq j \leq l(u), 1 \leq k \leq l(v)} 2^{i_{u, j}+i_{v, k}} .
$$

As the vertices that have 1 on the $i^{\text {th }}$ bit is a subset, this creates one biclique per pair of such subsets, for a total of $O\left(\log ^{2} n\right)$ bicliques, each including every vertex in the worst case.

Combining these with Lemma 7.7 then gives our algorithm for carrying one step of the Schur complement approximation algorithm.

Lemma 7.16. Given a graph $G$ with $n$ vertices and $m$ edges, we can produce an $\varepsilon$-graphic spectral sketch of the graph corresponding to the matrix on the RHS of Equation 9 with $n^{1+o(1)} \varepsilon^{-1}$ edges in $n^{1+o(1)} \varepsilon^{-1.5}+m n^{o(1)} \varepsilon^{-0.5}$ time.

Proof. As observed above, the RHS of Equation 9 is a sum of weighted cliques whose total number of vertices is $m$. Lemmas 7.14 and 7.15 allow us to decompose these into unit weighted bicliques.

Theorem A.2 further reduces the number of vertices involved in the graph representation of these bicliques. Specifically we obtain $\mathcal{K}_{B, 1}, \mathcal{K}_{B, 2} \ldots$ such that

$$
\sum_{i} n\left(\mathcal{K}_{B, i}\right) \leq \widetilde{O}(m)
$$

and each $\mathcal{K}_{B, i}$ has edge weights which are powers of 2.

Invoking Lemma 7.7 on each $\mathcal{K}_{B, i}$ gives an $\varepsilon$-spectral sketch of this graph with

$$
n^{1+o(1)} \epsilon^{-1.5}+m n^{o(1)} \epsilon^{-0.5}
$$


edges. Note that the guarantees of Lemma 7.7 are additive across a sum of graphs. Thus Lemma 6.8 gives the preservation of quadratic inverse forms in this approximation as well.

Sketching this graph explicitly using $\operatorname{SpectralSketch}(\cdot, \epsilon / 2$, ShortCycleDecomposition) as specified in Theorems 6.1 and 3.11 gives the final edge count and approximation guarantees. The running time follows from the size of this explicit graph that we sketch, as well as the guarantees of Lemma 7.7 .

Invoking this routine repeatedly as in $\left[\mathrm{KLP}^{+} 16\right]$, and then recursively within the effective resistance estimation routine from $\left[\mathrm{DKP}^{+} 17\right]$ gives the overall result for computing effective resistances.

Proof. (of Theorem 3.8) We first consider graphs whose weights are poly $(n)$, and therefore have condition number $\operatorname{poly}(n)$. An additional property of Schur complements is that they can only increase the minimum (non-zero) eigenvalue, and decrease the maximum (weighted) degree. This means as long as the initial eigenvalues are poly bounded, we can 'fix' the Schur complements after each step based on eigenvalues to ensure that the edge weights remain poly bounded while incurring a $1 / \operatorname{poly}(n)$ error at each step.

Lemma G.2 from $\left[\mathrm{KLP}^{+} 16\right]$ along with this poly(n) bound on condition number means that after $O(\log n)$ iterations of applying the transformation from Equation 9, the top-left (FF) block becomes negligible. This then means that the bottom right block is a resistance preserving sparsifier of $\operatorname{SC}(\boldsymbol{L}, C)$. This then fits into the requirement of Lemma 7.13 with parameters

$$
\begin{aligned}
\Delta(\widehat{n}, \widehat{\varepsilon}) & =\widehat{n}^{o(1)} \cdot \widehat{\varepsilon}^{-1} \\
T_{S C}\left(\widehat{n}, \widehat{m}, \widehat{\varepsilon}^{-1}\right) & =\widehat{n}^{1+o(1) \widehat{\varepsilon}^{-3 / 2}+\widehat{m}^{1+o(1)} \widehat{\varepsilon}^{-1 / 2}},
\end{aligned}
$$

which gives the overall running time. Note that we accumulate errors naively during this process, leading to additional polylog factors in the errors.

Finally, Appendix $\mathrm{F}$ of $\left[\mathrm{CKP}^{+} 17\right]$ provides a reduction from solving linear systems in arbitrary graph Laplacians to solving linear systems in graphs with poly(n) bounded edge weights. Applying it to resistances allows us to make the poly(n) bounded weights assumption as above, at the cost of another $O(\log n)$ overhead. Broadly speaking, such reductions are similar to those in Appendix $\mathrm{A}$ in that they remove/contract edges whose weights are smaller/larger than true estimates by poly $(n)$ factors, while incurring an error of $1 / \operatorname{poly}(n)$. We omit explicitly adapting this argument to the effective resistance case in anticipation of a more systematic and unified treatment of such reductions in the near future.

\section{Efficient Construction of Short Cycle Decomposition}

In this section, we give an almost-linear time algorithm to construct a short cycle decomposition of a graph, proving Theorem 3.11. We start with the existence proof (Theorem 3.10), which can also be phrased as an $O(n m)$ time algorithm.

Proof. (of Theorem 3.10). We first bound the running time: Each iteration of the outer loop takes $O(n)$ time since we stop constructing the BFS tree when we encounter the first non-tree edge. Since each iteration of the outer loop removes at least one edge from $G$, the overall running time is $O(m n)$. 


\begin{tabular}{l}
$\overline{\text { Algorithm 11 NAIVECYCLEDECOMPOSITION }(G)}$ \\
\hline Input: Undirected Unweighted Graph $G(V, E)$. \\
Output: $\mathcal{C}$, that's a $\left(2 n, 2 \log _{2} n\right)$ cycle decomposition.
\end{tabular}

1. Repeat until $G$ is empty.

(a) While $G$ has a vertex $u$ of degree $\leq 2$, remove $u$ and the edges incident to $u$ from $G$.

(b) Run breadth-first search (BFS) from an arbitrary vertex $r$ until you encounter the first non-tree edge.

(c) Let $C$ be the cycle formed by $e$ and the tree edges.

(d) Add $C$ to the collection of cycles, $\mathcal{C} \leftarrow \mathcal{C} \cup\{C\}$.

(e) Remove the edges of $C$ from $G, E(G) \leftarrow E(G) \backslash E(C)$.

2. Return $\mathcal{C}$.

For every vertex deleted, we delete at most 2 edges, and hence at most $2 n$ edges are not part of the cycles.

When running BFS, $G$ does not have any vertices of degree 1, and hence it cannot be a tree, and thus BFS must find a non-tree edge. It remains to bound the lengths of cycles, which is at most the depth of the first non-tree edge in the BFS tree T. Suppose this happens at a depth of $d$. Then every node at depth $d$ or higher has at least two children, for a total of at least $2^{d}$ vertices. Since the number of vertices remaining is at most $n$, we get $2^{d} \leq n$, or $d \leq \log _{2} n$.

We now turn to the almost-linear time cycle decomposition algorithm that produces longer cycles. Its pseudocode is presented as Algorithm 15, At a high level, its outline is as follows:

1. Decompose the graph into a set of disjoint expanders and a small number of edges.

2. In each expander, select a small set of high degree vertices and "port" a large number of the edges into these vertices via random walks.

3. Recurse on the ported edges on these smaller vertex sets.

\subsection{Porting Edges onto Fewer Vertices}

In this section, we describe our key routine that ports a large fraction of edges onto a smaller set of vertices $S$. The object that allow this movement and subsequent reconstruction can be formalized as a cycle decomposition of the graph $G / S$, the graph $G$ with the vertices of $S$ shrunk to a single vertex, which we'll denote as $s^{*}$.

Definition 8.1. Given a graph $G$ and a vertex subset $S \subseteq V$, a $(\widehat{l}, \widehat{k})$-partial cycle decomposition onto $S$ is a union of at least $\widehat{k}$ edge-disjoint cycles of length at most $\widehat{l}$ in $G / S$, the graph formed by shrinking $S$ to a single vertex $s^{*}$.

Such a partial cycle decomposition allows us to create a graph on $S$ such that any cycle decomposition of this smaller graph can be transformed into a cycle decomposition of $G$ with the same number of cycles, and a length overhead of $\widehat{l}$. 
Lemma 8.2. Given $G, S \subset V$, and a $(\widehat{l}, \widehat{k})$-partial cycle decomposition onto $S$, we can create a graph $G_{S}$ with at most $\widehat{k}$ edges such that any $(\widehat{m}, l)$-short cycle decomposition of $G_{S}$ can be transformed in $O(m)$ time into cycles on $G$ with length at most $l \cdot \widehat{l}$ that contain at least $\widehat{k}-\widehat{m}$ edges.

Proof. First, note that any cycle in $G / S$ that does not pass through $s^{*}$ is already a cycle in $G$.

We create $G_{S}$ as follows: for each cycle in the partial decomposition that passes through $s^{*}$ (the new vertex corresponding to $S$ ), let the edges incident to $s^{*}$ in this cycle be $e_{1}$ and $e_{2}$. These two edges are also then incident to vertices in $S, s_{1}$ and $s_{2}$. We add an edge between $s_{1}$ and $s_{2}$ in $G_{S}$.

This means any edge in $G_{S}$ has a path in $G$ between its two end points. Furthermore, the corresponding paths between all edges of $G_{S}$ are edge-disjoint. Then for any cycle in $G_{S}$, replacing each edge in $G_{S}$ with the corresponding path in $G$ completes it into a circuit in $G$, which we can then break into cycles. As all edges in this cycle decomposition of $G_{S}$ remain in these cycles, we get the lower bound on number of edges involved.

Our algorithm is then based on repeatedly generating such partial cycle decompositions containing a significant fraction of the edges, recursively finding cycle decompositions of the resulting graph $G_{S}$, and extending them back to cycles in $G$ using Lemma 8.2 above. It relies on the following key size reduction routine.

Lemma 8.3. Let $\gamma_{\mathrm{NS}}(n)=\exp (O(\sqrt{\log n \log \log n}))$ be the overhead term from expander decomposition routine (Lemma 8.7 [NS1\%]). Given a graph $G$ on $n$ vertices with $m$ edges and degrees between $\left[d_{\min }, d_{\max }\right]$, along with a reduction factor $k \geq 10 \log n$ and $k \leq n$ such that

$$
d_{\min } \geq 8000\left(\frac{d_{\max }}{d_{\min }}\right)^{2} \gamma_{\mathrm{NS}}^{3} k \log n
$$

the routine $\operatorname{Move} \operatorname{Edges}(G, k)$ runs in $O\left(m k\left(d_{\max } / d_{\min }\right)^{2} \gamma_{\mathrm{NS}}^{2} \log n\right)$ expected time, and returns a subset of at most $2 n / k$ vertices $S$ and $a$

$$
\left(400\left(\frac{d_{\max }}{d_{\min }}\right)^{2} \gamma_{\mathrm{NS}}^{2} \log n,\left(\frac{d_{\min }}{d_{\max }}\right)^{4} \cdot \frac{1}{10^{6} k \gamma_{\mathrm{NS}}^{4} \log ^{2} n} \cdot m\right)
$$

partial cycle decomposition of $G$ onto $S$.

Our notion of expanders is given by conductance. The conductance of a graph $G$ is:

$$
\phi(G):=\min _{S \subseteq V(G), 0<\operatorname{vol}(S) \leq m} \frac{\left|E_{G}(S, V \backslash S)\right|}{\operatorname{vol}(S)},
$$

where $\operatorname{vol}(S)$ is the total degrees of the vertices in $S$. A large conductance means that a random walk starting anywhere in the graph quickly approaches the uniform distribution. For our purposes, the role played by conductance can be summarized as:

Lemma 8.4. Let $G$ be an unweighted undirected simple graph with conductance $\phi$, and let $S$ be any subset of vertices. Then for any vertex $u$, and any random walk starting at $u$ continuing for $10 \phi^{-2} \log n$ steps is at a vertex in $S$ with probability at least $\frac{\operatorname{vol}(S)}{3 m}$. 
Proof. Let $A$ denote the adjacency matrix of $G$ and $D$ denote the diagonal degree matrix. The random walk matrix for the lazy walk on $G$ is given by $W=\frac{1}{2} I+\frac{1}{2} A D^{-1}$, and the normalized adjacency matrix $N=D^{-1 / 2}(D-A) D^{-1 / 2}$. Cheeger's inequality gives us $\lambda_{2}(N) \geq \phi^{2} / 2$. Thus,

$$
\max _{\lambda \neq 1} \lambda(W)=1-\lambda_{2}(I-W)=1-\frac{1}{2} \lambda_{2}(N) \leq 1-\phi^{2} / 4 .
$$

Thus, if we take a $k$-step lazy random walk in $G$ with $k=10 \phi^{-2} \log n$, we get, $\max _{\lambda \neq 1} \lambda\left(W^{k}\right) \leq$ $n^{-5 / 2}$. Thus, the probability that a $k$-step random walk from $u$ ends up at a vertex in $S$ is given by $\overrightarrow{1}_{S}^{\top} W^{k} \overrightarrow{1}_{u}$. In order to lower bound this, we observe that $D^{-1 / 2} W D^{1 / 2}$ is a symmetric matrix with the same eigenvalues as $W$ and thus with a largest eigenvalue of 1 (with eigenvector $D^{1 / 2} \overrightarrow{1}$ ). Thus,

$$
\begin{aligned}
\overrightarrow{1}_{S}^{\top} W^{k} \overrightarrow{1}_{u} & =\overrightarrow{1}_{S}^{\top} D^{1 / 2}\left(D^{-1 / 2} W D^{1 / 2}\right)^{k} D^{-1 / 2} \overrightarrow{1}_{u} \\
& \geq \overrightarrow{1}_{S}^{\top} D^{1 / 2}\left(\frac{1}{2 m} D^{1 / 2} \overrightarrow{1}^{\top} D^{1 / 2}\right) D^{-1 / 2} \overrightarrow{1}_{u}-\frac{1}{n^{5 / 2}}\left\|D^{1 / 2} \overrightarrow{1}_{S}\right\|\left\|D^{-1 / 2} \overrightarrow{1}_{u}\right\| \\
& \geq \frac{\operatorname{vol}(S)}{2 m}-\frac{1}{n^{5 / 2}} \sqrt{\frac{\operatorname{vol}(S)}{\operatorname{deg}(u)}} \geq \frac{\operatorname{vol}(S)}{3 m},
\end{aligned}
$$

for $n$ large enough.

Note that the $\log n$ factor in the length of the random walks is necessary, since there exist constant degree expander graphs (with $\phi=\Omega(1)$ ) where at least half the vertices are at a distance $\Omega(\log n)$ from any starting vertex $u$.

The above lemma prompts us to start with expanders. Algorithm 12 describes the pseudocode for this procedure.

Lemma 8.5. Given an unweighted undirected graph $G$ with $n$ vertices, $m$ edges, conductance at least $\phi$, for any parameter $k$ such that

$$
10 \log n \leq k \leq \frac{\phi^{2} d_{\min }}{100 \log n}
$$

where $d_{\min }$ is the minimum degree of a vertex in $G, \operatorname{MoveEdgEsExpander}(G, \phi, k)$ returns a graph on $\lceil n / k\rceil$ vertices along with a

$$
\left(25 \phi^{-2} \log n, \frac{\phi^{4} m}{2 \cdot 10^{3} k \log ^{2} n}\right)
$$

partial cycle decomposition of $G$ onto $S$, in expected running time $O\left(m k \phi^{-2} \log n\right)$.

We first prove that sampling random walks does not incur too much congestion.

Lemma 8.6. In any graph $G$, if we sample $k \geq 10 \log n$ random walks, each of length $L$, from both end points of each edge in $G$. The congestion on every edge $e$ is bounded by $4 k L$ with probability at least $1-\frac{1}{n}$. 
Algorithm 12 MoveEdgesExpander $(G, \phi, k)$

Input: Undirected unweighted graph $G=(V, E)$ with $n \geq k$ vertices and $m \geq n$ edges $\phi$, a lower bound on conductance of $G$

Reduction factor $k$.

Output: A set of $\lceil n / k\rceil$ vertices $S$

A partial cycle decomposition of $G$ onto $S$.

1. Pair up multiple edges to form cycles.

2. Pick $S$ consisting of the $\lceil n / k\rceil$ vertices of maximum degree in $G$.

3. For every edge $e=(u, v) \in E$,

(a) Generate $4 k$ lazy random walks each from $u$ and $v$ of length $10 \phi^{-2} \log n$. A lazy random walk stays at the current vertex with probability $1 / 2$ at each step.

(b) Discard walks that use $e$.

(c) Pick any one walk each from $u$ and $v$ (if there is one) that terminates in $S$ and convert them into simple paths. Add the corresponding cycle in $G / S$ to consideration.

4. Greedily pick a set of edges whose corresponding cycles are edge-disjoint.

5. If fewer than $\frac{\phi^{4} m}{2 \cdot 10^{3} k \log ^{2} n}$ cycles are formed, go to Step 3 ,

6. Return $S$, and the partial cycle decomposition

Proof. Orient the edges arbitrarily, and fix an edge $\hat{e}$. Let $X_{e, j, i}$ denote the probability that a single random walk starting at the $j^{\text {th }}$ end point of $e(j \in\{1,2\})$ goes through $\hat{e}$ at step $i$. Since the stationary distribution is uniform across all the edges, for all $i$, we have,

$$
\sum_{e} \sum_{j=1,2} \mathbb{E}\left[X_{e, j, i}\right]=2
$$

However, note that $X_{e, j, i}$ are not independent.

Thus, we define the random variable $X_{e}^{(j)}$ to be the congestion on $\hat{e}$ incurred by the $j^{\text {th }}$ length $L$ walks out of both end points of $e$. We then have $X_{e}^{(j)} \leq 2 L$, and the total congestion on $\hat{e}$ is $\sum_{e, j} X_{e}^{(j)}$ with the expectation

$$
\sum_{1 \leq j \leq k} \sum_{e \in E} \mathbb{E}\left[X_{e}^{(j)}\right] \leq 2 L k
$$

Since $X_{e}^{(j)}$ are independent for all $e, j$, and we have $k>\log n$, applying Hoeffding's bound, we obtain that the total congestion of $\hat{e}$ is bounded by $4 k L$ with probability at least $1-e^{-k / 3} \geq 1-n^{-3}$ with high probability.

Applying union bound over all edges $\hat{e}$, we obtain our claim.

Proof. (of Lemma 8.5) Since $S$ is the set of $n / k$ nodes with the highest degree, its volume is at least $\frac{2 m}{k}$. So by Lemma 8.4, the probability that a random walk on $G$ arrives at $S$ after $10 \phi^{-2} \log n$ steps is at least $\frac{2 m}{k} \cdot \frac{1}{3 m}=\frac{2}{3 k}$. 
Furthermore, since each vertex of $G$ has degree at least $100 \phi^{-2} k \log n$, this walk uses the edge $e$ with probability at most $\frac{1}{100 k \phi^{-2} \log n} \cdot 10 \phi^{-2} \log n=\frac{1}{10 k}$. Thus, the walk hits $S$ without using the edge $e$ with probability at least $\frac{2}{3 k}-\frac{1}{10 k} \geq \frac{1}{2 k}$.

Since we take $4 k$ random walks from each end point of $e$, this means that with probability at least $1-2 / e^{2} \geq \frac{3}{5}$, at least one of the walks from each end point of $e$ reaches $S$. Thus, in expectation, at least $\frac{3 m}{5}$ edges reach $S$, and the probability of fewer than $\frac{m}{2}$ edges reaching $S$ is at most $\frac{4}{5}$.

We now need to bound the number of disjoint cycles found. Lemma 8.6 proves that the congestion of each edge is at most $40 k \phi^{-2} \log n$ with high probability. Assuming this congestion bound holds, since each cycle uses at most $20 \phi^{-2} \log n$ edges (in addition to $e$ ), every cycle conflicts with at most $\left(1+20 \phi^{-2} \log n\right)\left(1+40 \phi^{-2} \log n\right)$ other ones. Thus, with probability at least $\frac{1}{10}$, we have at least

$$
\frac{m}{2} \cdot \frac{1}{\left(1+20 \phi^{-2} \log n\right)\left(1+40 k \phi^{-2} \log n\right)} \geq \frac{m \phi^{4}}{2 \cdot 10^{3} k \log ^{2} n}
$$

cycles, giving us our bound.

Lemma 8.5 gives a way to partially cycle decompose an expander, which suggests using it in conjunction with an expander decomposition scheme. However, most existing efficient expander decomposition schemes only provide clusters that are contained in expanders. Since our algorithm requires subgraphs where the random walks mix well, we need to restrict the random walks to expanders.

Instead we utilize a more result by Nanongkai and Saranurak [NS17] that guarantees an expander decomposition where the resulting subgraphs are expanders. This routine gives its guarantees in terms of edge expansion, $h(G)$, which can be defined as

$$
h(G)=\min _{S \subseteq V(G), 0<|S| \leq \frac{n}{2}} \frac{\left|E_{G}(S, V \backslash S)\right|}{|S|} .
$$

Lemma 8.7 (Theorem 5.1 from NS17] 10). There is an algorithm NSEXPANDERDECOMPOSE that for any undirected graph $G=(V, E)$ and parameter $\alpha>0$, partitions $E$ into $E^{s}$ and $E^{d}$ such that for $\gamma_{\mathrm{NS}}(n)=\exp (O(\sqrt{\log n \log \log n}))$,

1. $\left|E^{s}\right| \leq \alpha \gamma_{\mathrm{NS}}(n) n$, and,

2. with high probability, every connected component $H_{i}$ of $G^{d}=\left(V, E^{d}\right)$ is either a singleton or has edge expansion $h\left(H_{i}\right) \geq \alpha$.

The time taken by the algorithm is $O\left(m \gamma_{\mathrm{NS}}(n) \log n\right)$.

Note in particular that having edge expansion at least $\alpha$ implies that each vertex has degree at least $\alpha$. We can translate between edge expansion and conductance when the graphs have bounded degrees. This is the reason for the dependency on $d_{\max } / d_{\min }$ in Lemma 8.3. The following simple lemma allows us to convert an edge expansion bound into a conductance bound.

Lemma 8.8. If a graph $G$ with degree at most $d_{\max }$ has edge-expansion at least $\alpha$, then the conductance of $G$ is at least $\frac{\alpha}{d_{\max }}$.

\footnotetext{
${ }^{10}$ From Version 2 https://arxiv.org/pdf/1611.03745v2.pdf
} 
Proof. Consider any set of vertices $S$ in $G$ with vol $(S) \leq m$. Since the maximum degree in $G$ is $d_{\text {max }}$, we have $|S| \geq \frac{\operatorname{vol}(S)}{d_{\max }}$. If $|S| \leq n / 2$, then invoking the edge expansion bound of $G$ on $S$ we get,

$$
|E(S, V \backslash S)| \geq \alpha \cdot|S| \geq \frac{\alpha}{d_{\max }} \operatorname{vol}(S) .
$$

Otherwise, since vol $(V \backslash S) \geq m$, we have $\frac{n}{2} \geq|V \backslash S| \geq \frac{m}{d_{\max }}$. Thus,

$$
|E(S, V \backslash S)| \geq \alpha \cdot|V \backslash S| \geq \frac{\alpha}{d_{\max }} m \geq \frac{\alpha}{d_{\max }} \operatorname{vol}(S) \text {. }
$$

With this bound in mind, we can then given the overall routine for moving the edges. Its pseudocode is given in Algorithm 13,

Algorithm 13 MoveEdges $(G, k)$

Input: graph $G=(V, E)$ with $n$ vertices, $m$ edges, and degrees in range $\left[d_{\min }, d_{\max }\right]$. Vertex count reduction parameter $k$.

access to parameter that's expander partitioning overhead $\gamma_{\mathrm{NS}}(n)$ from Lemma 8.7.

Output: A set of at most $2 n / k$ vertices $S$ and a partial cycle decomposition of $G$ onto $S$.

1. Set $\alpha \leftarrow d_{\min } / 4 \gamma_{\mathrm{NS}}(n)$

2. $\left(E^{s}, E^{d}\right) \leftarrow \operatorname{NSExpanderDeCompose}(G, \alpha)$.

3. For each connected component $H_{i}$ of $E^{s}$

(a) If $\left|V\left(H_{i}\right)\right| \leq k$, record the cycles generated using $\operatorname{NaiveCyCleDeComposition}\left(H_{i}\right)$.

(b) Else record the result of $\operatorname{MoveEdgesExpander}\left(H_{i}, \alpha / d_{\max }, k\right)$.

4. Return the unions of the subsets and partial cycle decompositions onto them.

Proof. (of Lemma 8.3) By guarantees of the expander decomposition given by Lemma 8.7, the number of edges contained in the expanders, $E^{d}$, is at least

$$
m-\alpha \gamma_{\mathrm{NS}}(n) n \geq m-\frac{d_{\min }}{4} n \geq \frac{m}{2}
$$

where we used our choice of $\alpha=\frac{d_{\min }}{4 \gamma_{\mathrm{NS}}(n)}$, and $n d_{\min } \leq 2 m$. Moreover, we know that for any $H_{i}$ such that $\left|V\left(H_{i}\right)\right| \geq 2$, the edge-expansion of each $H_{i}$ is at least $\alpha$, and hence the degree of each vertex in $H_{i}$ is at least $\alpha$.

For each $H_{i}$ with at least 2 and at most $k$ vertices, we call NaiveCyCleDeComposition, resulting in an amortized a total running time of at most $O\left(k\left|E\left(H_{i}\right)\right|\right)$. In each $H_{i}$, this results in at least

$$
\left|E\left(H_{i}\right)\right|-2\left|V\left(H_{i}\right)\right| \geq\left|E\left(H_{i}\right)\right|-4 \alpha^{-1}\left|E\left(H_{i}\right)\right| \geq \frac{1}{2}\left|E\left(H_{i}\right)\right|,
$$

edges being incorporated into cycles of length at most $2 \log n$, which means at least $\left|E\left(H_{i}\right)\right| / 4 \log n$ cycles. 
Lemma 8.8 gives that the conductance of each $H_{i}$ is at least

$$
\phi=\frac{\alpha}{d_{\max }}=\frac{d_{\min }}{4 d_{\max } \gamma_{\mathrm{NS}}(n)}
$$

and every vertex has degree at least

$$
\alpha=\frac{d_{\min }}{4 \gamma_{\mathrm{NS}}(n)} \geq 8000\left(\frac{d_{\max }}{d_{\min }}\right)^{2} \gamma_{\mathrm{NS}}(n)^{3} k \log n \cdot \frac{1}{4 \gamma_{\mathrm{NS}}(n)}=100 \phi^{-2} k \log n .
$$

Thus, for $H_{i}$ with $\left|V\left(H_{i}\right)\right| \geq k$, Lemma 8.5 gives that the number of cycles produced in the partial decomposition is at least

$$
\left(\frac{d_{\min }}{d_{\max }}\right)^{4} \frac{\left|E\left(H_{i}\right)\right|}{512 \cdot 10^{3} \gamma_{\mathrm{NS}}(n)^{4} k \log ^{2} n}
$$

and the expected running time is $O\left(\left|E\left(H_{i}\right)\right| k\left(d_{\max } / d_{\min }\right)^{2} \gamma_{\mathrm{NS}}(n)^{2} \log n\right)$. Combining these bounds over all connected components of $H_{i}$ then gives the bounds on running time and edges moved.

The bound on the lengths of the cycles in this partial cycle decomposition also follows from

$$
25 \phi^{-2} \log n \leq 400\left(\frac{d_{\max }}{d_{\min }}\right)^{2} \gamma_{\mathrm{NS}}(n)^{2} \log n .
$$

\subsection{Recursive Cycle Decomposition}

Now that we have decreased the number of vertices by a large fraction $k$, while decreasing the edge counts accordingly, we simply recurse on this smaller subproblem. This results in about $m / k$ edges being placed into cycles in the original parent problem, which in turn implies about $k$ iterations of recursion involving problems of size about $m / k$. Such a trade-off works well with the Master theorem, except we need to handle the recursion explicitly due to the overhead of $\gamma_{\mathrm{NS}}(n)$ at each iteration.

In particular, Lemma 8.2 gives that any cycle on the smaller graph translates to a cycle on the original graph that's are longer by a factor of $\operatorname{poly}\left(\gamma_{\mathrm{NS}}(n), \log n\right)=n^{o(1)}$. This means we can afford such multiplicative blowups a constant, or sub-logarithmic number of times throughout the course of the algorithm.

However, one technical issue that we need to address first is the reduction from arbitrary degree graphs to ones with uniform degrees. Note that the high degree vertices may be concentrated among a small number of vertices. We will repeatedly peel away the low degree vertices, from which we can obtain a graph where every vertex has degree at least $\Delta$ assigned to it. This graph may in turn have vertices with high degrees: for example, a star can have 1 edge assigned to each vertex, but a center vertex with degree $n-1$. We address this by splitting those vertices up into multiple vertices: cycles in the resulting graph are still cycles in the original graph. Pseudocode of this routine is in Algorithm 14,

Note that as we will call this procedure repeatedly, we will have the procedure of repeatedly removing low degree vertices in the outer loop in order to amortize its cost over all the removals.

The following lemma helps us to convert the graph into one with bounded degrees. 


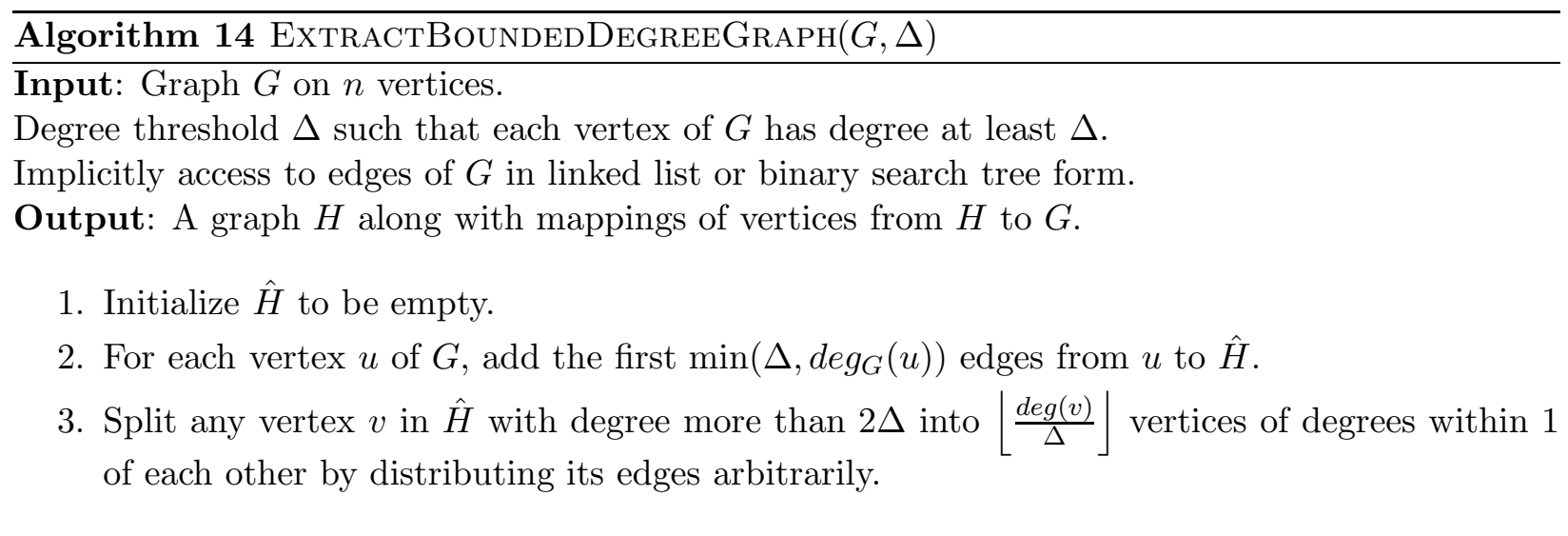

Lemma 8.9. Given a graph $G$ on $n$ vertices with every vertex having degree at least $\Delta$, the procedure ExtractBoundedDegreeGraph $(G, \Delta)$ returns in $O(n \Delta)$ time a graph $H$ such that:

1. $H$ has at most $2 n$ vertices.

2. Every vertex in $H$ has degrees in the range $[\Delta, 2 \Delta]$.

3. Every cycle in $H$ corresponds to a circuit in $G$.

Proof. The assumption of minimum degree at least $\Delta$ in $G$, and the addition of first $\Delta$ neighbors to $H$ ensures that $\hat{H}$ has minimum degree at least $\Delta$, and at most $\Delta n$ edges. This also means the total degree in $\hat{H}$ is at most $2 \Delta n$.

The splitting process takes linear time, and ensures that all degrees in $H$ are in the range $[\Delta, 2 \Delta]$. Combining this with the total degree also means that $H$ has at most $2 n$ vertices.

The splitting process maintains a bijection between the edges of $G$ and $H$, and also ensures that all the edges incident on a vertex $u_{H}$ in $H$ are also incident to the original copy of that vertex in $G$. Thus every cycle in $H$ can be mapped to a circuit in $G$.

Repeatedly calling this and the edge moving procedure from Section 8.1 then leads to our overall algorithm, whose pseudocode is in Algorithm 15.

We first give the guarantees of this algorithm in terms of its output and the total sizes of recursive problems that it invokes:

Lemma 8.10. For any $l \geq 0$, any $k \geq 10 \log n$, and any graph $G$ with $n$ vertices and $m$ edges, invoking CycleDecomposition $(G, l, k)$ returns $\mathcal{C}$ and $E_{\text {extra }}$ so that the number edges in $E_{\text {extra }}$ is at most

$$
\left(64 \cdot 10^{6} \gamma_{\mathrm{NS}}(n)^{4} \log ^{2}(2 n)\right)^{l} k n,
$$

and the length of cycles in $\mathcal{C}$ is at most $\left(2000 \gamma_{\mathrm{NS}}(n)^{2} \log (2 n)\right)^{l+1}$.

The total running time cost is bounded by $O(m n)$ if $l=0$, and otherwise

1. $O\left(m k \gamma_{\mathrm{NS}}(n)^{2} \log n\right)$ in the cost of preprocessing and creating the $H_{S} s$,

2. Recursive calls to CyCleDecomposition $\left(H_{S}, l-1, k\right)$ where the total edge count in $H_{S}$ across all steps is at most $2 m$, and each $H_{S}$ has at most $4 n / k$ vertices. 


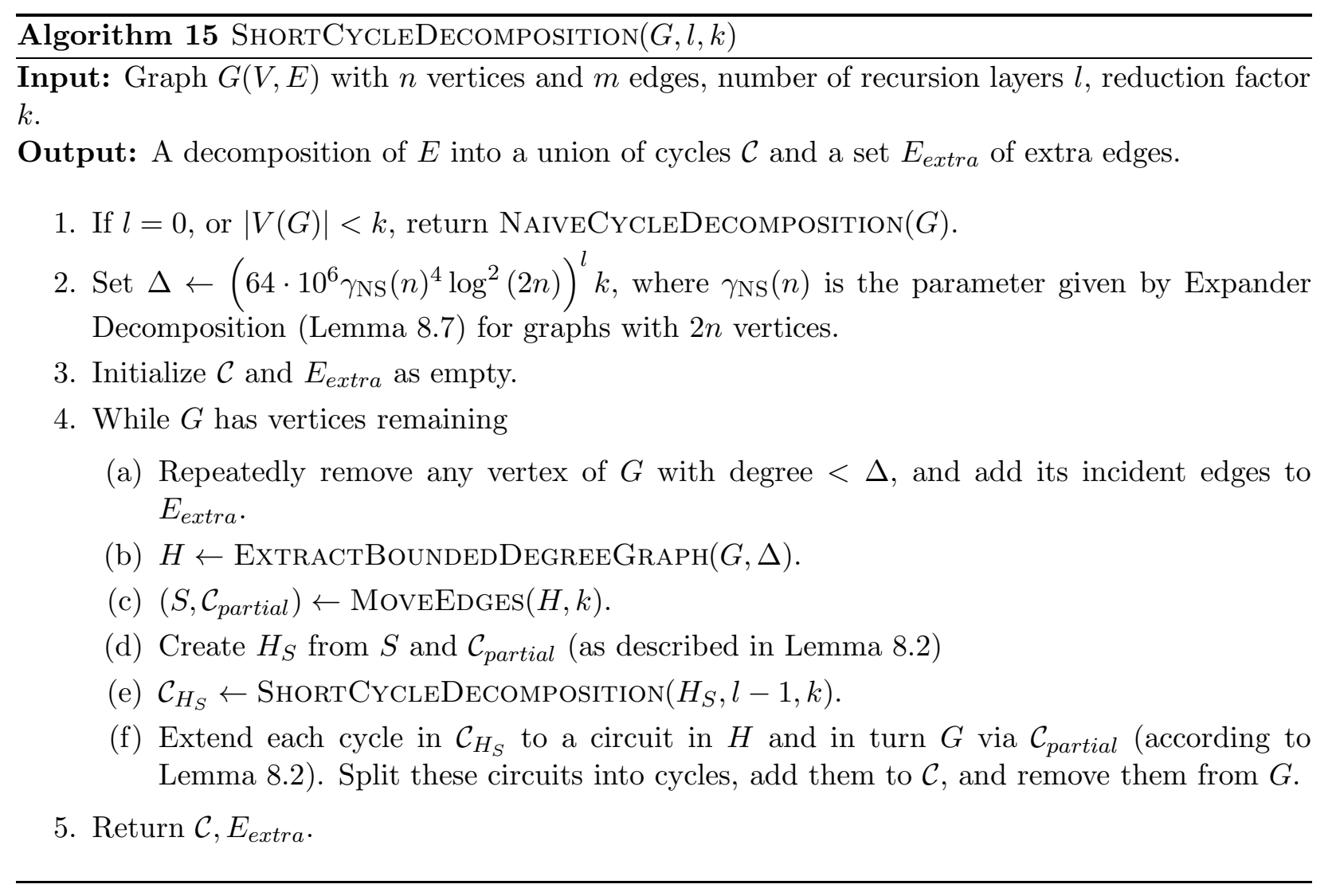


Proof. If $l=0$, it follows from Theorem 3.10 that the running time is bounded by $O(m n)$. Otherwise, if $|V(G)| \leq k$, again by Lemma 3.10 the running time is bounded by $O(m k)=$ $O\left(m k \gamma_{\mathrm{NS}}(n)^{2} \log n\right)$ as desired. In either case, the size of $E_{\text {extra }}$ is bounded by $2 n \leq k n$, and the length of the cycles is at most $2 \log n$, giving all the required guarantees in the case we call NAIVECYCLEDECOMPOSITION.

Otherwise, the size of $E_{\text {extra }}$ follows since the only edges added to $E_{\text {extra }}$ are from vertices with degree at most $\Delta$.

Lemma 8.9 gives that the minimum and maximum degrees of $H$ are within a factor of 2 , and it has at most $2 n$ vertices. So Lemma 8.3 gives that the lengths of the cycles in $\mathcal{C}_{\text {partial }}$ is at most $2000 \gamma_{\mathrm{NS}}(n)^{2} \log (2 n)$. This and Lemma 8.2 means that any cycle $\mathcal{C}_{\hat{G}}$ corresponds to a cycle in $G$ whose length is longer by a factor of $2000 \gamma_{\mathrm{NS}}(n)^{2} \log (2 n)$. Conditioning on the recursion terminating, and applying induction with the guarantees of Lemma 3.10 as the base case gives the bounds on lengths of cycles returned.

It remains to bound the running time, which we do by inductively showing that each recursive call makes significant call relative to the work done. The base case of $l=0$ has been argued above. For the inductive case, assume the result is true for $l-1$. Consider one iteration of the while loop, and say $\hat{n} \leq n$ is the number of vertices remaining in $G$ at the start of this iteration. The choice of $\Delta$ means that $H$ satisfies the degree requirement of Lemma 8.3 and has at most $2 \hat{n}$ vertices. Thus, $H_{S}$ has at most $4 \hat{n} / k$ vertices, and the number of cycles found is at least

$$
\frac{1}{16 \cdot 10^{6} k \gamma_{\mathrm{NS}}(n) \gamma_{\mathrm{NS}}(n)^{4} \log ^{2}(2 n)}|E(H)| \geq \frac{1}{16 \cdot 10^{6} k \gamma_{\mathrm{NS}}(n)^{4} \log ^{2}(2 n)} \Delta \hat{n},
$$

Substituting in the value of $\Delta$ then gives this is at least

$$
\frac{4}{k} \cdot\left(64 \cdot 10^{6} \gamma_{\mathrm{NS}}(n)^{4} \log ^{2}(2 n)\right)^{l-1} k \hat{n} .
$$

While by the inductive hypothesis, the number of extra edges returned by the recursive call to CycleDecomposition $(\hat{G}, l-1, k)$ is at most

$$
\left(64 \cdot 10^{6} \gamma_{\mathrm{NS}}(n)^{4} \log ^{2}(2 n)\right)^{l-1} k \frac{2 \hat{n}}{k}
$$

so at least half of the edges of $H_{S}$ are now incorporated into the extended cycles given by Lemma 8.2 , This means that each edge we examined can be 'charged' to the edges added to the cycles at a cost of 2 edges examined per edge added to $\mathcal{C}$. Since each edge can only be added to $\mathcal{C}$ once, the total sizes of the recursive calls is bounded by $2 m$.

The total preprocessing costs now follow from the running time for MoveEDGEs given by Lemma 8.3. Thus, the inductive hypothesis holds for $l$ as well.

The above lemma shows that the progress ShortCyCleDeComposition makes per recursive call is close to the number of edges in that problem. Note that the progress made at the steps can be highly non-uniform: a $\sqrt{n}$-sized clique in an otherwise sparse graph will result in most of the recursive calls happening on $\sqrt{n}$ sized graphs, with about $\sqrt{n}$ edges moved in each, while the first few iterations may be removing about $n$ edges each.

Setting parameters in the above lemma then gives the proof of Theorem 3.11 , the main result of this section. 
Proof. (Of Theorem [3.11) We consider setting parameters in Lemma 8.10, The lengths of the cycles constructed is $\left(2000 \gamma_{\mathrm{NS}}(n)^{2} \log (2 n)\right)^{l+1}$. Since $\gamma_{\mathrm{NS}}(n)=\exp (O(\sqrt{\log n \log \log n}))$, picking $l=(\log n)^{1 / 4}$, we get that the length of the cycles is

$$
\exp \left(O\left((\log n \log \log n)^{1 / 2}\right)\right)^{(\log n)^{1 / 4}+O(1)}=\exp \left(O\left((\log n \log \log n)^{3 / 4}\right)\right)=n^{o(1)}
$$

Next, we bound the total time taken by the procedure. First, we count the total cost of constructing $\hat{G}$ 's across all $l$ levels of recursion with size reduction factor $k$. At each recursion level, we invoke ShortCycleDecomposition with a total of twice as many edges. The time required for the construction at a level with $m$ input edges is $O\left(m k \gamma_{\mathrm{NS}}(n)^{2} \log n\right)$, resulting in a total construction time of

$$
O\left(2^{l} m k \gamma_{\mathrm{NS}}(n)^{2} \log n\right) \text {. }
$$

There is also the cost all the calls to NaiveCycleDecomposition. The calls that arise at the bottom recursion level $(l=0)$ result in a total running time of $O\left(m n(4 / k)^{l}\right)$. For all calls that to NAiveCyCleDeComposition that arise since $|V(\hat{G})| \leq k$, the cumulative running time is bounded by $O\left(2^{l} m k\right)$.

Thus, the total running time taken by $\operatorname{ShortCyCleDecomposition}(G, l, k)$ is bounded by

$$
O\left(2^{l} m k \gamma_{\mathrm{NS}}(n)^{2} \log n+m n(4 / k)^{l}\right) .
$$

In order to balance the two terms, we pick $k^{l}=n$, and thus $k=\exp \left((\log n)^{3 / 4}\right)$, giving a total running time of

$$
m \exp \left(O\left((\log n)^{3 / 4}\right)\right)=m^{1+o(1)} .
$$

Finally, the only edges not in cycles are those in $E_{\text {extra }}$ at the top level of recursion, and the total number of such edges is bounded by

$$
\left(64 \cdot 10^{6} \gamma_{\mathrm{NS}}(n)^{4} \log ^{2}(2 n)\right)^{l} k n=n \exp \left(O\left((\log n)^{3 / 4}\right)\right)=n^{1+o(1)}
$$

\section{Acknowledgments}

We thank John Peebles for many insightful discussions, David Durfee for pointing out several key details in the interaction with determinant estimation from Appendix $\mathrm{B}$, and Di Wang for explaining to us the state-of-the-art for efficiently generating expander decompositions.

\section{References}

[ACK $\left.{ }^{+} 16\right]$ Alexandr Andoni, Jiecao Chen, Robert Krauthgamer, Bo Qin, David P Woodruff, and Qin Zhang. On sketching quadratic forms. In Proceedings of the 2016 ACM Conference on Innovations in Theoretical Computer Science, pages 311-319. ACM, 2016. Available at: https://arxiv.org/abs/1511.06099. 2, 5, 9, 11, 173 
[ALO15] Zeyuan Allen Zhu, Zhenyu Liao, and Lorenzo Orecchia. Spectral sparsification and regret minimization beyond matrix multiplicative updates. In Proceedings of the Forty-Seventh Annual ACM on Symposium on Theory of Computing, STOC 2015, Portland, OR, USA, June 14-17, 2015, pages 237-245, 2015. [5

[AM85] N Alon and V.D Milman. Isoperimetric inequalities for graphs, and superconcentrators. Journal of Combinatorial Theory, Series B, 38(1):73 - 88, 1985. 32

[AP90] Baruch Awerbuch and David Peleg. Sparse partitions (extended abstract). In 31st Annual Symposium on Foundations of Computer Science, St. Louis, Missouri, USA, October 22-24, 1990, Volume II, pages 503-513, 1990. 12

[ASW18] Sepehr Assadi, Xiaorui Sun, and Omri Weinstein. Massively parallel algorithms for finding well-connected components in sparse graphs. CoRR, abs/1805.02974, 2018. Available at: http://arxiv.org/abs/1805.02974. 13

[BK96] András A. Benczúr and David R. Karger. Approximating s-t minimum cuts in $\tilde{O}\left(n^{2}\right)$ time. In Proceedings of the twenty-eighth annual ACM symposium on Theory of computing, STOC '96, pages 47-55, New York, NY, USA, 1996. ACM. 2, 5

[BK16] Greg Bodwin and Sebastian Krinninger. Fully dynamic spanners with worst-case update time. CoRR, abs/1606.07864, 2016. Available at: http://arxiv.org/abs/1606.07864. 13

[BKS12] Surender Baswana, Sumeet Khurana, and Soumojit Sarkar. Fully dynamic randomized algorithms for graph spanners. ACM Transactions on Algorithms (TALG), 8(4):35, 2012. 12

[BR11] Aaron Bernstein and Liam Roditty. Improved dynamic algorithms for maintaining approximate shortest paths under deletions. In Proceedings of the twenty-second annual ACM-SIAM symposium on Discrete Algorithms, pages 1355-1365. Society for Industrial and Applied Mathematics, 2011. 12

[BS08] Surender Baswana and Soumojit Sarkar. Fully dynamic algorithm for graph spanners with polylogarithmic update time. In Proceedings of the Nineteenth Annual ACM-SIAM Symposium on Discrete Algorithms, SODA 2008, San Francisco, California, USA, January 20-22, 2008, pages 1125-1134, 2008. 12

[BSS12] Joshua Batson, Daniel A Spielman, and Nikhil Srivastava. Twice-Ramanujan sparsifiers. SIAM Journal on Computing, 41(6):1704-1721, 2012. 2] 5

[BSST13] Joshua Batson, Daniel A. Spielman, Nikhil Srivastava, and Shang-Hua Teng. Spectral sparsification of graphs: theory and algorithms. Communications of the ACM, 56(8):87-94, August 2013. 3, 5]

[CCL $\left.{ }^{+} 15\right]$ Dehua Cheng, Yu Cheng, Yan Liu, Richard Peng, and Shang-Hua Teng. Efficient sampling for Gaussian graphical models via spectral sparsification. Proceedings of The 28th Conference on Learning Theory, pages 364-390, 2015. Available at http://jmlr.org/proceedings/papers/v40/Cheng15.pdf. 47

[Che86] P Chew. There is a planar graph almost as good as the complete graph. In Proceedings of the Second Annual Symposium on Computational Geometry, SCG '86, pages 169-177, New York, NY, USA, 1986. ACM. 2

$\left[\mathrm{CKM}^{+} 11\right]$ Paul Christiano, Jonathan A. Kelner, Aleksander Madry, Daniel A. Spielman, and ShangHua Teng. Electrical flows, laplacian systems, and faster approximation of maximum flow in undirected graphs. In Proceedings of the 43rd annual ACM symposium on Theory of 
computing, STOC '11, pages 273-282, New York, NY, USA, 2011. ACM. Available at http://arxiv.org/abs/1010.2921. 10

$\left[\mathrm{CKP}^{+} 16\right]$ Michael B Cohen, Jonathan Kelner, John Peebles, Richard Peng, Aaron Sidford, and Adrian Vladu. Faster algorithms for computing the stationary distribution, simulating random walks, and more. In Foundations of Computer Science (FOCS), 2016 IEEE 57th Annual Symposium on, pages 583-592. IEEE, 2016. Available at: https://arxiv.org/abs/1608.03270. 3] 7, 19

$\left[\mathrm{CKP}^{+} 17\right]$ Michael B Cohen, Jonathan Kelner, John Peebles, Richard Peng, Anup B Rao, Aaron Sidford, and Adrian Vladu. Almost-linear-time algorithms for markov chains and new spectral primitives for directed graphs. In Proceedings of the 49th Annual ACM SIGACT Symposium on Theory of Computing, pages 410-419. ACM, 2017. Available at: https://arxiv.org/abs/1611.00755. 78. 20, 47, 56

[CKST17] Charles Carlson, Alexandra Kolla, Nikhil Srivastava, and Luca Trevisan. Optimal lower bounds for sketching graph cuts. CoRR, abs/1712.10261, 2017. Available at: https://arxiv.org/abs/1712.10261. 2

[CL06] Fan Chung and Linyuan Lu. Concentration inequalities and martingale inequalities: a survey. Internet Mathematics, 3(1):79-127, 2006. Available at: http://www.math.ucsd.edu/ ${ }^{\sim}$ fan/wp/concen.pdf. 30

[DFGX18] David Durfee, Matthew Fahrbach, Yu Gao, and Tao Xiao. Nearly tight bounds for sandpile transience on the grid. In SODA, pages 605-624. SIAM, 2018. Available at: https://arxiv.org/abs/1704.04830. 10.

[DGGP18] David Durfee, Yu Gao, Gramoz Goranci, and Richard Peng. Fully dynamic effective resistances. CoRR, abs/1804.04038, 2018. Available at: http://arxiv.org/abs/1804.04038. 13

$\left[\mathrm{DKP}^{+} 17\right]$ David Durfee, Rasmus Kyng, John Peebles, Anup B Rao, and Sushant Sachdeva. Sampling random spanning trees faster than matrix multiplication. In Proceedings of the 49th Annual ACM SIGACT Symposium on Theory of Computing, pages 730-742. ACM, 2017. Available at: https://arxiv.org/abs/1611.07451. 2, 3, 4, 10, 11, 53, 56,

[DKW15] Michael Dinitz, Robert Krauthgamer, and Tal Wagner. Towards Resistance Sparsifiers. In Naveen Garg, Klaus Jansen, Anup Rao, and José D. P. Rolim, editors, Approximation, Randomization, and Combinatorial Optimization. Algorithms and Techniques (APPROX/RANDOM 2015), volume 40 of Leibniz International Proceedings in Informatics (LIPIcs), pages 738-755, Dagstuhl, Germany, 2015. Schloss Dagstuhl-Leibniz-Zentrum fuer Informatik. 3

[DPPR17] David Durfee, John Peebles, Richard Peng, and Anup B. Rao. Determinant-preserving sparsification of SDDM matrices with applications to counting and sampling spanning trees. In FOCS, pages 926-937. IEEE Computer Society, 2017. Available at: https://arxiv.org/abs/1705.00985. 3. 4, 10, 12, 53, 78, 79, 80,

[EGIN97] David Eppstein, Zvi Galil, Giuseppe F Italiano, and Amnon Nissenzweig. Sparsification: a technique for speeding up dynamic graph algorithms. Journal of the ACM (JACM), 44(5):669696, 1997. 2

$\left[\mathrm{FMP}^{+} 17\right]$ Matthew Fahrbach, Gary L. Miller, Richard Peng, Saurabh Sawlani, Junxing Wang, and Shen Chen Xu. On computing min-degree elimination orderings. CoRR, abs/1711.08446, 2017. 38

[GSY17] Jie Gao, Grant Schoenebeck, and Fang-Yi Yu. Cascades and myopic routing in nonhomogeneous kleinberg's small world model. In WINE, volume 10660 of Lecture Notes in Computer Science, pages 383-394. Springer, 2017. 13 
[JS18] Arun Jambulapati and Aaron Sidford. Efficient $\widetilde{O}(n / \varepsilon)$ spectral sketches for the laplacian and its pseudoinverse. In SODA, pages 2487-2503. SIAM, 2018. Available at: https://arxiv.org/abs/1711.00571. 2, 4, 5, 9, 11, 29, 32, 38, 73,

[KLOS14] Jonathan A. Kelner, Yin Tat Lee, Lorenzo Orecchia, and Aaron Sidford. An almost-linear-time algorithm for approximate max flow in undirected graphs, and its multicommodity generalizations. In Proceedings of the Twenty-Fifth Annual ACM-SIAM Symposium on Discrete Algorithms, SODA 2014, Portland, Oregon, USA, January 5-7, 2014, pages 217-226, 2014. Available at http://arxiv.org/abs/1304.2338. 13, 33

[KLP12] Ioannis Koutis, Alex Levin, and Richard Peng. Improved Spectral Sparsification and Numerical Algorithms for SDD Matrices. In Christoph Dürr and Thomas Wilke, editors, 29th International Symposium on Theoretical Aspects of Computer Science (STACS 2012), volume 14 of Leibniz International Proceedings in Informatics (LIPIcs), pages 266-277, Dagstuhl, Germany, 2012. Schloss Dagstuhl-Leibniz-Zentrum fuer Informatik. Available at http://arxiv.org/abs/1209.5821. 5, 10, 19, 27

[KLP $\left.{ }^{+} 16\right]$ Rasmus Kyng, Yin Tat Lee, Richard Peng, Sushant Sachdeva, and Daniel A Spielman. Sparsified cholesky and multigrid solvers for connection laplacians. In Proceedings of the 48 th Annual ACM SIGACT Symposium on Theory of Computing, pages 842-850. ACM, 2016. Available at http://arxiv.org/abs/1512.01892. 2, 11, 47, 53, 54, 56,

[KM11] Jonathan A. Kelner and Petar Maymounkov. Electric routing and concurrent flow cutting. Theor. Comput. Sci., 412(32):4123-4135, 2011. Available at: https://arxiv.org/abs/0909.2859. 13

[KMP11] Ioannis Koutis, Gary L. Miller, and Richard Peng. A nearly-m log n time solver for SDD linear systems. In Proceedings of the 2011 IEEE 52nd Annual Symposium on Foundations of Computer Science, FOCS '11, pages 590-598, Washington, DC, USA, 2011. IEEE Computer Society. Available at http://arxiv.org/abs/1102.4842. 2

[KMP14] I. Koutis, G. Miller, and R. Peng. Approaching optimality for solving sdd linear systems. SIAM Journal on Computing, 43(1):337-354, 2014. Available at http://arxiv.org/abs/1003.2958. 2

[Kou14] Ioannis Koutis. Simple parallel and distributed algorithms for spectral graph sparsification. In Proceedings of the 26th ACM Symposium on Parallelism in Algorithms and Architectures, SPAA '14, pages 61-66, New York, NY, USA, 2014. ACM. Available at http://arxiv.org/abs/1402.3851. 5

[KP12] Michael Kapralov and Rina Panigrahy. Spectral sparsification via random spanners. In Proceedings of the 3rd Innovations in Theoretical Computer Science Conference, ITCS '12, pages 393-398, New York, NY, USA, 2012. ACM. Available at http://people.csail.mit.edu/kapralov/papers/spanners.pdf. [5]

[KPPS17] Rasmus Kyng, Jakub Pachocki, Richard Peng, and Sushant Sachdeva. A framework for analyzing resparsification algorithms. In Proceedings of the Twenty-Eighth Annual ACMSIAM Symposium on Discrete Algorithms, pages 2032-2043. SIAM, 2017. Available at: https://arxiv.org/abs/1611.06940. 5

[KS16] Rasmus Kyng and Sushant Sachdeva. Approximate gaussian elimination for laplacians - fast, sparse, and simple. In FOCS, pages 573-582. IEEE Computer Society, 2016. Available at http://arxiv.org/abs/1605.02353. 53

[Kyn17] Rasmus Kyng. Approximate Gaussian Elimination. PhD thesis, Yale University, 2017. Available at: http://rasmuskyng.com/rjkyng-dissertation.pdf. 7 
[Lov93] László Lovász. Random walks on graphs: A survey. Combinatorics, Paul Erdős is Eighty, 2:1-46, 1993. 10

[LPS88] A. Lubotzky, R. Phillips, and P. Sarnak. Ramanujan graphs. Combinatorica, 8(3):261-277, 1988. 12

[LPS $\left.{ }^{+} 18\right]$ Huan Li, Richard Peng, Liren Shan, Yuhao Yi, and Zhongzhi Zhang. Current flow group closeness centrality for complex networks. CoRR, abs/1802.02556, 2018. Available at: https://arxiv.org/abs/1802.02556. 2

[LS15] Yin Tat Lee and He Sun. Constructing linear-sized spectral sparsification in almost-linear time. In IEEE 56th Annual Symposium on Foundations of Computer Science, FOCS 2015, Berkeley, CA, USA, 17-20 October, 2015, pages 250-269, 2015. Available at: https://arxiv.org/abs/1508.03261. 5

[LS17] Yin Tat Lee and He Sun. An SDP-based algorithm for linear-sized spectral sparsification. In Proceedings of the 49th Annual ACM SIGACT Symposium on Theory of Computing, STOC 2017, Montreal, QC, Canada, June 19-23, 2017, pages 678-687, 2017. Available at: https://arxiv.org/abs/1702.08415. [5]

[LZ18] Huan Li and Zhongzhi Zhang. Kirchhoff index as a measure of edge centrality in weighted networks: Nearly linear time algorithms. In Proceedings of the Twenty-Ninth Annual ACMSIAM Symposium on Discrete Algorithms, pages 2377-2396. SIAM, 2018. Available at: https://arxiv.org/abs/1708.05959. 2

[Mad10] Aleksander Madry. Fast approximation algorithms for cut-based problems in undirected graphs. In Foundations of Computer Science (FOCS), 2010 51st Annual IEEE Symposium on, pages 245-254. IEEE, 2010. Available at http://arxiv.org/abs/1008.1975. 13

[MGKT15] Charalampos Mavroforakis, Richard Garcia-Lebron, Ioannis Koutis, and Evimaria Terzi. Spanning edge centrality: Large-scale computation and applications. In $W W W$, pages $732-742$. ACM, 2015. Available at: https://www.cs.cmu.edu/ jkoutis/papers/spanning_edge.pdf. 10

[MNS $\left.{ }^{+} 18\right]$ Cameron Musco, Praneeth Netrapalli, Aaron Sidford, Shashanka Ubaru, and David P. Woodruff. Spectrum approximation beyond fast matrix multiplication: Algorithms and hardness. In ITCS, volume 94 of LIPIcs, pages 8:1-8:21. Schloss Dagstuhl - Leibniz-Zentrum fuer Informatik, 2018. Available at: https://arxiv.org/abs/1704.04163. 10]

[MST15] Aleksander Madry, Damian Straszak, and Jakub Tarnawski. Fast generation of random spanning trees and the effective resistance metric. In Proceedings of the Twenty-Sixth Annual ACMSIAM Symposium on Discrete Algorithms, SODA 2015, pages 2019-2036, 2015. Available at http://arxiv.org/pdf/1501.00267v1.pdf. 10

[NS17] Danupon Nanongkai and Thatchaphol Saranurak. Dynamic spanning forest with worst-case update time: adaptive, las vegas, and $o\left(n^{1 / 2-\epsilon}\right)$-time. In Proceedings of the 49th Annual ACM SIGACT Symposium on Theory of Computing, STOC 2017, Montreal, QC, Canada, June 19-23, 2017, pages 1122-1129, 2017. Available at: https://arxiv.org/abs/1611.03745. 13, 58, 61

[Pen16] Richard Peng. Approximate undirected maximum flows in $O(m$ polylog $(n))$ time. In Proceedings of the Twenty-Seventh Annual ACM-SIAM Symposium on Discrete Algorithms, pages 18621867. SIAM, 2016. Available at http://arxiv.org/abs/1411.7631. 13

[PS14] Richard Peng and Daniel A. Spielman. An efficient parallel solver for SDD linear systems. In Proceedings of the 46th Annual ACM Symposium on Theory of Computing, STOC '14, pages 333-342, New York, NY, USA, 2014. ACM. Available at http://arxiv.org/abs/1311.3286. 2, 47 
[Rac08] Harald Racke. Optimal hierarchical decompositions for congestion minimization in networks. In Proceedings of the 40th annual ACM symposium on Theory of computing, STOC '08, pages 255-264, New York, NY, USA, 2008. ACM. 13

[RST14] Harald Racke, Chintan Shah, and Hanjo Taubig. Computing cut-based hierarchical decompositions in almost linear time. In Proceedings of the 25th Annual ACM-SIAM Symposium on Discrete Algorithms, SODA '14, pages 227-238, 2014. 13

[Sar10] Purnamrita Sarkar. Tractable algorithms for proximity search on large graphs. Carnegie Mellon University, 2010. Available at: http://www.cs.cmu.edu/ ${ }^{\sim}$ psarkar/thesis/sarkar_thesis.pdf. 10

[Sch17] Aaron Schild. An almost-linear time algorithm for uniform random spanning tree generation. CoRR, abs/1711.06455, 2017. Available at: https://arxiv.org/abs/1711.06455. 3] 10, 12

[She13] Jonah Sherman. Nearly maximum flows in nearly linear time. In 54th Annual IEEE Symposium on Foundations of Computer Science, FOCS 2013, 26-29 October, 2013, Berkeley, CA, USA, pages 263-269, 2013. Available at http://arxiv.org/abs/1304.2077. 13

[SM07] Purnamrita Sarkar and Andrew W Moore. A tractable approach to finding closest truncatedcommute-time neighbors in large graphs. In Proceedings of the Twenty-Third Conference on Uncertainty in Artificial Intelligence, pages 335-343. AUAI Press, 2007. Available at: https://arxiv.org/abs/1206.5259. 10

[Spi10] Daniel A. Spielman. Algorithms, Graph Theory, and Linear Equations in Laplacian Matrices. In Proceedings of the International Congress of Mathematicians, 2010. Available at: www.cs.yale.edu/homes/spielman/PAPERS/icm10post.pdf. 3

[SS11] D. Spielman and N. Srivastava. Graph sparsification by effective resistances. SIAM Journal on Computing, 40(6):1913-1926, 2011. Available at http://arxiv.org/abs/0803.0929. 2, 3, 4, 5, 6] 10, 19, 27, 73

[ST11a] D. Spielman and S. Teng. Spectral sparsification of graphs. SIAM Journal on Computing, 40(4):981-1025, 2011. Available at http://arxiv.org/abs/0808.4134. 13

[ST11b] Daniel A. Spielman and Shang-Hua Teng. Spectral sparsification of graphs. SIAM Journal on Computing, 40(4):981-1025, 2011. Available at http://arxiv.org/abs/0808.4134. 2, 5, 9 , 32, 33

[ST14] D. Spielman and S. Teng. Nearly linear time algorithms for preconditioning and solving symmetric, diagonally dominant linear systems. SIAM Journal on Matrix Analysis and Applications, 35(3):835-885, 2014. Available at http://arxiv.org/abs/cs/0607105. 2

[Ten10] Shang-Hua Teng. The Laplacian Paradigm: Emerging Algorithms for Massive Graphs. In Theory and Applications of Models of Computation, pages 2-14, 2010. 3

[Ten16] Shang-Hua Teng. Scalable algorithms for data and network analysis. Foundations and Trends in Theoretical Computer Science, 12(1-2):1-274, 2016. Available at: http://wwwbcf.usc.edu/ shanghua/teaching/Fall2017-670/networkDataAnalysisPrintedBook.pdf. 3

[Tro12] Joel A. Tropp. User-friendly tail bounds for sums of random matrices. Found. Comput. Math., 12(4):389-434, August 2012. Available at http://arxiv.org/abs/1004.4389. 14, 15, 20, 21, 38

[Val82] Leslie G. Valiant. A scheme for fast parallel communication. SIAM journal on computing, 11(2):350-361, 1982. 13 


\section{A Reduction to Unit Weight Case}

We briefly describe how to reduce a general weighted graph to a sum of graphs, each with edges of the same weight. This reduction underlies the constructions of spectral sparsifiers [SS11], as well as previous results on graph sketching using expander decompositions [ACK ${ }^{+} 16$, JS18]. The main idea stems from the observation that if $u$ is connected to a vertex $x$ by an edge of weight 1 , and $x$ in turn has a path to $y$ with each edge having weight at least $\operatorname{poly}(n)$, then the edge $u x$ can be replaced by the edge $u y$ while incurring a negligible error of $1 / \operatorname{poly}(n)$. Such an observation plus bucketing of edge weights then allows one to 'move' the end points of lower weighted edges incident to some highly weighted component to a single vertex in such a component. For such edges to not become self-loops, they must exit the component. This in turn reduces the number of connected components as we move to lower weighted edge classes. As a result, the number of vertices can be bounded by the decrease in the number of connected components, which is at most $n$.

Because our reduction only incurs $1 / \operatorname{poly}(n)$ error, we can give a unified treatment of undirected and directed graphs. This is via a definition of almost equality that's analogous to the with high probability (w.h.p.) notation for accumulating 1/poly $(n)$ small failure probabilities. Because an undirected edge is the sum of two directed edges, one in each direction, we will define this notation in the more general case of directed graphs from Theorem 5.1 .

Definition A.1. Two (algorithmically generated) (directed) graphs $\vec{G}$ and $\vec{H}$ are almost equal if for any constant $\delta>0$, we can adjust constants in our algorithms so that

$$
\left\|\boldsymbol{L}_{G}^{+/ 2}\left(\boldsymbol{L}_{\vec{G}}-\boldsymbol{L}_{\vec{H}}\right) \boldsymbol{L}_{G}^{+/ 2}\right\| \leq n^{-\delta} .
$$

We will denote this using $\vec{G} \cong \vec{H}$.

Here, $\boldsymbol{L}_{G}$ and $\boldsymbol{L}_{\vec{G}}$ are defined as in section 5 , with $\boldsymbol{L}_{G}$ defined in Equation 5 , Our result is a black box reduction among graphs that keeps them almost equal. The conditions that we keep are stronger than both degree-preserving sparsification from Section 4 and Eulerian sparsification from Section 5. We want to preserve both the in and out degrees exactly, instead of just their differences.

Theorem A.2. Any Eulerian directed graph $\vec{G}$ with arbitrary weights on $n$ vertices and $m$ edges, represented either explicitly, or implicitly as a sum of bicliques, can be decomposed in nearly-linear time into a sum of a graph $\vec{H}_{\text {sparse }}$ with $O(n \log n)$ edges plus graphs $\vec{H}_{1}, \vec{H}_{2}, \ldots$ such that

$$
\vec{G} \cong \vec{H}_{\text {sparse }}+\sum_{i} \vec{H}_{i}
$$

and,

1. The graph $\vec{H}_{\text {sparse }}+\sum \vec{H}_{i}$ has the exact same in/out weighted degrees at each vertex as in $\vec{H}$.

2. All edge weights in each $\vec{H}_{i}$ are powers of 2 .

3. The total number of vertices in $\left\{\vec{H}_{i}\right\}$ is $O\left(n \log ^{2} n\right)$.

4. The total number of edges in $\left\{\vec{H}_{i}\right\}$ is $O(m \log n)$.

5. If $\vec{G}$ is represented implicitly as a sum of bicliques, the total representation size of $\vec{H}_{i}$ returned is larger by a factor of $O\left(\log ^{2} n\right)$. 
Because we only work with $1 / \operatorname{poly}(n)$ sized perturbations, we will pick $\vec{H}_{\text {sparse }}$ from maxweighted spanning trees of the undirected graph $G$. This choice is convenient for aggregating the resulting changes in in/out degrees along the tree, and fixing them with paths along the tree.

Lemma A.3. For any directed graph $\vec{G}$, choosing $T$ to be $1 /$ poly $(n)$ times any subgraph of $G$, the undirected version of $\vec{G}$, gives

$$
\vec{G}+T \cong \vec{G}
$$

Proof. Let $\widehat{T}$ be any subgraph of $G$. Thus,

$$
\left\|\boldsymbol{L}_{G}^{+/ 2} \boldsymbol{L}_{\widehat{T}} \boldsymbol{L}_{G}^{+/ 2}\right\| \leq 1
$$

and the result follows from the rescaling.

For the case with implicitly represented bicliques, we mean that each vertex $u$ has weight $w_{u}$, and an edge $u v$ has weight $w_{u} \cdot w_{v}$ - and thus the biclique can be represented by two sets of vertices and vertex weights. Note that the max weight spanning tree of a weighted biclique is given by the two stars centered at the vertices with maximum weights. So this computation still takes time nearly-linear in the number of vertices involved in such a representation.

As we need to preserve degrees exactly, it is preferably to only make adjustments along even length cycles. We do so by restricting to bipartite graphs. This is done by decompositions similar to the random bipartition picked in the degree-preserving sparsifiers and sketches in Algorithms 2 and 5. However, in our case, we apply this as a preprocessing step to decompose into bipartite graphs completely, instead of iteratively removing edges from a bipartition that captures at least half the edges at a time.

Lemma A.4. Any directed graph $\vec{G}$ can be written as a sum of directed graphs $\vec{G}_{1}, \vec{G}_{2} \ldots$ so that

1. For each $i$, the undirected support of $\vec{G}_{i}, G_{i}$ is bipartite.

2. The total number of edges in $\left\{\vec{G}_{i}\right\}$ is $m$.

3. The the total number of vertices in $\left\{\vec{G}_{i}\right\}$ is $O(n \log n)$.

Such a decomposition takes $O(m)$ time if $\vec{G}$ is given explicitly, or $O\left(n\left(\mathcal{K}_{B}\right)\right)$ time if it's specified implicitly as a sum of bicliques. Furthermore, in the latter case, the number of vertices in the resulting cliques is $O\left(n\left(\mathcal{K}_{B}\right) \log n\right)$.

Proof. A greedy bipartition captures at least half of the edges, after which we can recursively decompose the edges in the two halves. Since the number of remaining edges halves after each step, we finish in $O(\log n)$ steps, and the total bound on the number of vertices follows from the recursive calls on the two halves being vertex disjoint.

In the case of implicit representations as bicliques, the bound follows from the fact that the intersection of a biclique with a bipartition is a biclique. Moreover, the restriction to the two sides gives two bicliques on the boundary of the bipartition with disjoint vertex sets.

Our proof then becomes modifying the graph in ways that introduce error significantly less than the weight of this tree added. Our most important lemma is a statement for 'locally' moving around the end point of an edge, but by a distance of 2 along the tree.

Lemma A.5. Suppose $u, x_{1}, x_{2}, x_{3}$ are four vertices in a directed graph $\vec{G}$ such that: 
- There is a directed edge of weight 1 from u to $x_{1}$

- The weights of the directed edges between $x_{1} x_{2}, x_{2} x_{3}$ in both directions exceed poly $(n)$.

Then the graphs $H$ produced by:

- removing weight 1 from $u \rightarrow x_{1}$ and $x_{2} \rightarrow x_{3}$,

- adding in $u \rightarrow x_{3}$ and $x_{2} \rightarrow x_{1}$,

has the same in/out degrees as $\vec{G}$, and satisfies $H \cong G$.

The same bound also holds in the case of an edge in the other direction from $x_{1}$ to $u$, but with the operations

- removing weight 1 from $x_{1} \rightarrow u$, and $x_{3} \rightarrow x_{2}$,

- adding in $x_{3} \rightarrow u$ and $x_{1} \rightarrow x_{2}$.

Proof. The difference produced is the matrix

$$
\left[\begin{array}{cccc}
0 & 0 & 0 & 0 \\
1 & 0 & -1 & 0 \\
0 & 0 & 0 & 0 \\
-1 & 0 & 1 & 0
\end{array}\right]
$$

where the vertices are arranged in the order $u, x_{1}, x_{2}, x_{3}$. This equals $\chi_{x_{1} x_{3}} \boldsymbol{\chi}_{u x_{2}}^{\top}$, and as the weight in $G$ between $x_{1} x_{2}$ and $x_{2} x_{3}$ are both poly $(n)$, we have

$$
\left\|\boldsymbol{L}_{G}^{+/ 2} \chi_{x_{1} x_{2}}\right\| \leq \sqrt{\boldsymbol{\chi}_{x_{1} x_{2}}^{\top} \boldsymbol{L}_{G}^{+} \boldsymbol{\chi}_{x_{1} x_{2}}} \leq 1 / \operatorname{poly}(n) .
$$

and similarly $\left\|\boldsymbol{L}_{G}^{+/ 2} \boldsymbol{\chi}_{x_{2} x_{3}}\right\| \leq 1 / \operatorname{poly}(n)$. Thus, by triangle inequality,

$$
\left\|\boldsymbol{L}_{G}^{+/ 2} \boldsymbol{\chi}_{x_{1} x_{3}}\right\| \leq\left\|\boldsymbol{L}_{G}^{+/ 2} \boldsymbol{\chi}_{x_{1} x_{2}}\right\|+\left\|\boldsymbol{L}_{G}^{+/ 2} \boldsymbol{\chi}_{x_{2} x_{3}}\right\| \leq 1 / \operatorname{poly}(n) .
$$

Since $u x_{1}$ is an edge in $G$ with weight 1, we have

$$
\left\|\boldsymbol{L}_{G}^{+/ 2} \boldsymbol{\chi}_{u x_{1}}\right\| \leq \sqrt{\boldsymbol{\chi}_{u x_{1}}^{\top} \boldsymbol{L}_{G}^{+} \boldsymbol{\chi}_{u x_{1}}} \leq 1
$$

and hence by triangle inequality,

$$
\left\|\boldsymbol{L}_{G}^{+/ 2} \boldsymbol{\chi}_{u x_{2}}\right\| \leq\left\|\boldsymbol{L}_{G}^{+/ 2} \boldsymbol{\chi}_{u x_{1}}\right\|+\left\|\boldsymbol{L}_{G}^{+/ 2} \boldsymbol{\chi}_{x_{1} x_{2}}\right\| \leq 1+1 / \operatorname{poly}(n) .
$$

Combining the above bounds with the Cauchy-Schwarz inequality then gives

$$
\left\|\boldsymbol{L}_{G}^{+/ 2} \boldsymbol{\chi}_{x_{1} x_{3}} \boldsymbol{\chi}_{u x_{2}}^{\top} \boldsymbol{L}_{G}^{+/ 2}\right\|=\left|\boldsymbol{\chi}_{u x_{2}} \boldsymbol{L}_{G}^{+} \boldsymbol{\chi}_{x_{1} x_{3}}\right| \leq\left\|\boldsymbol{L}_{G}^{+/ 2} \boldsymbol{\chi}_{u x_{2}}\right\|\left\|\boldsymbol{L}_{G}^{+/ 2} \boldsymbol{\chi}_{x_{1} x_{3}}\right\| \leq 1 / \operatorname{poly}(n) .
$$

The case with the other direction follows from the difference being the matrix

$$
\left[\begin{array}{cccc}
0 & 0 & 0 & 0 \\
-1 & 0 & 1 & 0 \\
0 & 0 & 0 & 0 \\
1 & 0 & -1 & 0
\end{array}\right]
$$

which is exactly the negation of the above matrix. 
This lemma essentially allows us to 'contract' higher weighted edges: an edge of weight $w$ can be moved along paths of weight exceeding $w \cdot \operatorname{poly}(n)$ while incurring negligible errors. In particular, it plus the condition of the undirected support being bipartite allows us to remove the trailing bits of the edge weights by canceling them along the maximum weighted spanning tree.

Lemma A.6. Any directed graph $\vec{G}$ whose undirected support is connected can be written as a sum of a directed graph $\vec{T}$ and several directed graphs $\vec{H}_{i}$ such that:

- The total number of edges in $\vec{T}$ and $\left\{\vec{H}_{i}\right\}$ is $O(m \log n)$.

- The undirected support of $\vec{T}$ is a tree.

- The in/out degrees in $\vec{G}$ and $\sum_{i} \vec{H}_{i}+\vec{T}$ are the same.

- All edge weights in $\vec{H}_{i}$ are $2^{i}$.

- $\vec{G} \cong \vec{T}+\sum_{i} \vec{H}$.

Furthermore, such a decomposition can be generated in $O(m \log n)$ time if $\vec{G}$ is given explicitly, or $O\left(\left(n\left(\mathcal{K}_{B}\right)+m\right) \log n\right)$ if we're given bicliques $\mathcal{K}_{B}$ instead.

Proof. We start by letting $\vec{T}$ be the maximum weight spanning tree in $G$, the undirectification of $\vec{G}$, scaled by $1 / \operatorname{poly}(n)$. Observe that we still treat $\vec{T}$ as a directed graph, with edges of equal weight in either direction. Lemma A.3 allows us to add $\vec{T}$ to $\vec{G}$ while incurring an error of $1 / \operatorname{poly}(n)$.

We now form the graphs $\vec{H}_{i}$ s by taking the first $O(\log n)$ leading bits of each edge's weights in $\vec{G}$. This gives

$$
\vec{G}=\vec{G}_{\text {trailing }}+\sum_{i} \vec{H}_{i}
$$

where the total number of edges in $\vec{H}_{i}$ is $O(m \log n)$ by construction. The constant factor in $O(\cdot)$ is chosen so that each edge in $\vec{G}_{\text {trailing }}$ has weight less than $1 / \operatorname{poly}(n)$ times the least weight of any edge on the corresponding directed path in $\vec{T}$.

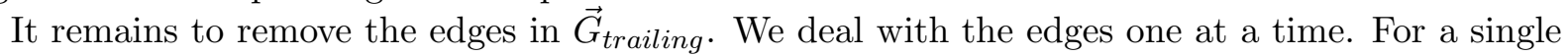
edge in $\vec{G}_{\text {trailing, }}$, we use Lemma A.5 repeatedly so as to locally move one of the end points of the edge closer to the other (in terms of the hop distance in $T$ ), along the unique path in $T$ between the end points of this edge. Specifically:

- By Lemma A.5, each such step incurs error that's at most $1 / \operatorname{poly}(n)$.

- Because $G$, and hence $T$ is bipartite, we can repeat this until the end points are distance 1 apart in $T$.

- This then coincides with an edge of $T$, so we can incorporate this edge in $\vec{T}$ by adjusting the edge weights in $\vec{T}$ appropriately, and discarding the original edge entirely.

Effectively this process 'reroutes' an edge in $\vec{G}_{\text {trailing }}$ along the unique path in $T$ connecting its end points.

Firstly, observe that both the local-move steps, and the tree-merge steps do not change the in and out degrees in the sum graph. Thus, the in/out degrees of all vertices are preserved.

It remains to show that this removal process keeps the graph almost equal. Due to the compounding of almost equality, we can analyze this one edge at a time. As there are at most $m \leq n^{2}$ edges, and each edge incurs at most $n$ local-move steps, the overall perturbation caused to the 
graph is still $1 / \operatorname{poly}(n)$. Thus, we can apply this process repeatedly until all edges in $\vec{G}_{\text {trailing }}$ are gone. At that point, we are left with $\vec{T}$ (with new, adjusted edge weights), plus the graph $\vec{H}_{i}$ whose total edge count is $O(m \log n)$.

Note that due to the adjustment in weights, the tree $\vec{T}$ is no longer undirected (or even Eulerian). However, the weights of the edges in $\vec{T}$ have only been perturbed by a multiplicative $1+1 / \operatorname{poly}(n)$ factors.

In the case where all the edges are explicitly given, this procedure can be implemented efficiently using dynamic trees. In the case of working with implicit representations, we perform recursive centroid decomposition on $\vec{T}$, after which the discrepancies only need to be propagated up to root. Then the amount propagated up to root can be calculated explicitly at each vertex, and then accumulated using a single depth-first search. In the case of given implicit representations as bicliques, the changes in degrees caused by the rounding can still be calculated explicitly, so routing the degree differences along $\vec{T}$ still remains the same.

Then it remains to 'shrink' the vertex set of each edge class (all edges with the same weight that's a power of 2). Note that the polynomially bounded weights case corresponds to there being $O(\log n)$ edge classes, at which point the result follows. We partition these edge classes into $O(\log n)$ buckets, where the $i^{\text {th }}$ bucket has edges of weight $2^{c_{\text {spread }} j \log n+i}$, for some positive integer $j$ and a some constant $c_{\text {spread }}$. Thus, we have split our graph into $O(\log n)$ graphs such that in each of these graphs, two edges either have the same weight, or their weights are apart by a factor more than $n^{c_{\text {spread }} \text {. }}$

On such a graph, Lemma A.5 enables us to 'move' the end points of a lower weighted edge along paths connected by a higher weighted class almost for free. This enables us to 'shrink' each connected component to two vertices connected in $\vec{T}$, and move all lower weighted edges to them. It in turn implies that all remaining edges (that are not absorbed into $\vec{T}$ are between components, giving a bound related to the reduction in the number of connected components of $\vec{T}$ as we introduce edge classes.

However, as Lemma A.5 only allows moving by two edges at a time, we still need to have two representative vertices per such connected component. This however does not affect the overall sizes across the edge classes.

Proof. (of Theorem A.2) Lemma A.4 allows us to reduce to the bipartite case with an overhead of $O(\log n)$, and Lemma A.6 allows us to work with a graph that's a sum of $H_{i}$ s, where each $H_{i}$ contains edges with weights $2^{i}$. The fact that we start with at most $O\left(n^{2}\right)$ edges also means that the number of non-empty $H_{i} \mathrm{~s}$ is at most poly $(n)$ (assuming the weights are at most $\exp (\operatorname{poly}(n))$.

We will create further separation between the edge weight classes by bucketing the $H_{i}$ s. For a constant $\xi \geq \Theta(\log n)$ that we will choose later, we will let the $j^{\text {th }}$ class include all indices with

$$
i \equiv j \quad(\bmod \xi)
$$

that is, the buckets containing edge weights

$$
2^{l \cdot \xi+j}
$$

for integers $l$. This introduces an additional overhead of $\xi=O(\log n)$ in the number of buckets, but ensures that two edges from the same bucket have weights that are either the same, or apart by a factor of $\operatorname{poly}(n)$. 
This $\operatorname{poly}(n)$ separation within each bucket ensures a clear separation by edge weights within each bucket. Thus we can perform the following shrinkage procedure:

1. For indices $i \equiv j(\bmod \xi)$ in decreasing order

(a) For each connected component of $G / H_{i} / H_{i+\xi} / H_{i+2 \xi} \ldots$,

i. Pick two representative vertices, one per side of the bipartition.

ii. Move the end points of each edge in $H_{i}$ to the representative vertex in its connected component of $G / H_{i} / H_{i+\xi} / H_{i+2 \xi} \ldots$ that's on the same side of its bipartition using Lemma A.5.

iii. Remove any self loops.

To efficiently implement this move, observe that this algorithm is identical to Kruskal's algorithm for computing a maximum weighted spanning tree in the $j^{\text {th }}$ edge bucket. So we can build the maximum spanning tree of the $j^{\text {th }}$ edge bucket, $\vec{T}^{(j)}$ before the loop. The changes to degrees caused by A.5 can then be computed in the same way as Lemma A.6, either implicitly or explicitly. As the lengths of these moves is $O(n)$, Lemma A.5 and the choice of $\xi \geq \Omega(\log n)$ gives a total error of $1 / \operatorname{poly}(n)$.

So it remains to bound the total number of vertices that each $H_{i}$ is moved to. Here the critical fact that we utilize is that a graph without self loops with edges incident to $\hat{n}$ vertices has at most $\hat{n} / 2$ connected components. This is because the lack of self loops means each connected component involves at least two vertices.

Then we can bound the number of vertices that $H_{i}$ gets moved to by the total number of connected components. Specifically, consider the potential function:

$$
\Phi(i):=\text { number of connected component in } G / H_{i} / H_{i+\xi} / H_{i+2 \xi} \cdots
$$

Now suppose the edges of $H_{i}$ after moving and removal of self loops are incident to $t(i)$ different components of $G / H_{i+\xi} / H_{i+2 \xi} / H_{i+3 \xi} \ldots$ Then contracting these edges decreases the number of connected components by at least $t / 2$, or formally

$$
\Phi(i) \leq \Phi(i+\xi)-t(i) / 2 .
$$

As $\Phi(\cdot)$ is between $[1, n]$, this implies a bound of $O(n)$ on the sum of $t(i)$ per edge bucket, for a total of $O(n \log n)$ vertices among all edge classes after the moves.

Putting back the overhead of $O(\log n)$ from bucketing on the weight classes, and another overhead of $O(\log n)$ from the decomposition to bipartite support then gives the overall bound.

\section{B Faster Determinant Estimation Using Faster Resistance Esti- mation}

We provide a short sketch showing how our results lead to the faster determinant estimation routine from Corollary 3.9, The algorithm utilizes the faster resistance estimation procedure from Theorem [3.8, and uses the machinery in the algorithm from Durfee et. al. [DPPR17] with slight tweaks on the parameters in that paper. The detailed references that we make below are all w.r.t version 1 of the same, which is available at https://arxiv.org/pdf/1705.00985.pdf. 
We start by tightening the parameters of the approximate Schur complement algorithm of [DPPR17]. Its pseudocode is given in Algorithm 4 in that paper, and its guarantees are in Theorem 5.3. The two key terms are:

1. $s$, the number of edges sampled.

2. $\varepsilon_{E R}$, the accuracy to which effective resistances on the edges need to be sampled.

and the tradeoffs given by these bounds for a variance of at most $\delta$ in the determinant estimation algorithm is given in Theorem 4.1. The guarantee on the parameters in Theorem 5.3 for approximate Schur complements can be summarized as

$$
\frac{\varepsilon_{E R}^{2} n^{2}}{s}+O\left(\frac{n^{3}}{s^{2}}\right) \leq \delta .
$$

Based on this tradeoff, we can tighten these parameters as below. The procedure has one additional technical restriction. It can only work when the vertices to be removed are 1.1-diagonally-dominant (1.1-DD): every vertex has at least $10 \%$ of its weighted degree leaving the set. Our modifications do not affect this step, so we only state it for completeness in the claims below.

Lemma B.1. There is a procedure SCHURSPARSE that takes a graph $G$ with $n$ vertices, $m$ edges, a 1.1-DD subset of vertices $V_{2}$, and error $\delta>0$, returns a graph $H^{V_{1}}$ with $O\left(n^{1.5+o(1)} \delta^{-0.5}\right)$ edges in $O\left(m^{1+o(1)}+n^{1.875+o(1)} \delta^{-0.875}\right)$ expected time such that the distribution over $H^{V_{1}}$ satisfies:

$$
\exp (-\delta) \operatorname{det}\left(\boldsymbol{L}_{\mathrm{SC}\left(G, V_{1}\right),-n}\right) \leq \underset{H^{V 1}}{\mathbb{E}}\left[\operatorname{det}\left(\boldsymbol{L}_{H^{V_{1},-n}}\right)\right] \leq \exp (\delta) \operatorname{det}\left(\boldsymbol{L}_{\mathrm{SC}\left(G, V_{1}\right),-n}\right)
$$

and

$$
\underset{H^{V 1}}{\mathbb{E}}\left[\operatorname{det}\left(\boldsymbol{L}_{H^{V_{1},-n}}\right)^{2}\right] \leq \exp (\delta) \underset{H^{V 1}}{\mathbb{E}}\left[\operatorname{det}\left(\boldsymbol{L}_{H^{V_{1},-n}}\right)\right]^{2} .
$$

Proof. Consider the parameter tradeoffs as discussed above just before the statement of this lemma. We will set $s$, the number of edges sampled to

$$
s=n^{1.5} \delta^{-0.5},
$$

which in turn necessitates computing their effective resistances to multiplicative accuracy

$$
\varepsilon_{E R}=n^{-0.25} \delta^{0.25}
$$

plugging these into Theorem 3.8 gives that we can compute the effective resistance of all $t=$ $n^{1.5} \delta^{-0.5}$ edges to this accuracy in time

$$
m^{1+o(1)}+n^{1.5} \delta^{-0.5} n^{o(1)}\left(n^{-0.25} \delta^{0.25}\right)^{-1.5}=m^{1+o(1)}+n^{1.875+o(1)} \delta^{-0.875} .
$$

The result then follows from incorporating the $\widetilde{O}(m)$ overhead in other steps from Theorem 4.1 of [DPPR17]. 
We remark that this new choice of parameters does not improve the runtime of the algorithm in [DPPR17] using previous algorithms for estimating effective resistances.

This change in turn propagates into the recursive algorithm as outlined in Algorithm 6 of [DPPR17]. This algorithm repeatedly finds 1.1-DD subsets that contain a constant fraction of vertices, and recurses on both the Schur complement and the matrix minor consisting of the removed vertices. A key difference between this algorithm and the recursive resistance approximation routine from Section 7.3 is that the overall determinant is a product of the determinants of the two subproblems. This means errors on them both accumulate into the overall error. This is addressed in [DPPR17] by letting the allowed variance vary with problem sizes. Below we outline how the better Schur complement sparsifier from Lemma B.1 can be readily incorporated to give an improved overall bound.

Proof. (of Corollary [3.9) In this proof, we use the notation found on page 30 and 31 of [DPPR17]. Consider setting the allowed variance onto a graph Schur complemented onto vertex set $V_{1}(i)$ as:

$$
\delta^{\prime}=\frac{\delta\left|V_{1}(i)\right|}{n}
$$

This is exactly the same as the second to last equation on page 31 of [DPPR17], so the overall error guarantees still hold.

Lemma B.1 gives that the cost of computing this is:

$$
\begin{gathered}
O\left(\left|V_{1}(i)\right|^{1.875+o(1)}\left(\delta^{\prime}\right)^{-0.875}\right)=O\left(\left|V_{1}(i)\right|^{1.875+o(1)}\left(\frac{\left|V_{1}(i)\right|}{n}\right)^{-0.875} \delta^{-0.875}\right) \\
=\left|V_{1}(i)\right| \cdot n^{0.875+o(1)} \delta^{-0.875}
\end{gathered}
$$

plus an initial overhead of $m^{1+o(1)}$. This initial overhead term is not present in subsequent layers of the recursion due to the vertex-based bound on edge count in Lemma B.1. The fact that the vertex count decreases by a constant factor at each step of the recursion outlined in [DPPR17] page 31 means that an instance on $V_{1}(i)$ has edge count bounded by

$$
\left|V_{1}(i)\right|^{1.5} \delta^{-0.5}
$$

So the overall cost at each level of the recursion is still bounded by

$$
\sum_{i}\left|V_{1}(i)\right| \cdot n^{0.875+o(1)} \delta^{-0.875} \leq n^{1.875+o(1)} \delta^{-0.875}
$$

which, summed over the $O(\log n)$ layers of recursion, gives the total. Note that we need to set $\delta=\varepsilon^{2}$ to obtain a $1 \pm \varepsilon$ approximation with constant probability. 